\title{
Transition Parameters for Doubly Ionized Lanthanum
}

\author{
Betuil Karaçoban and Leyla Özdemir \\ Department of Physics, Sakarya University, 54187 Sakarya, Turkey \\ Correspondence should be addressed to Betül Karaçoban, bkaracoban@sakarya.edu.tr
}

Received 6 July 2012; Accepted 8 August 2012

Academic Editor: Alan Migdall

Copyright (C) 2012 B. Karaçoban and L. Özdemir. This is an open access article distributed under the Creative Commons Attribution License, which permits unrestricted use, distribution, and reproduction in any medium, provided the original work is properly cited.

\begin{abstract}
The transition parameters such as the wavelengths, weighted oscillator strengths, and transition probabilities (or rates) for the nd $(n=5-9)-\operatorname{nf}(n=4-8)$, nd $(n=5-9)-\mathrm{np}(n=6-9), \mathrm{np}(n=6-9)-\mathrm{ns}(n=6-10)$, and $\mathrm{ng}(n=5-8)-\mathrm{nf}(n=4-8)$ electric dipole (E1) transitions of doubly ionized lanthanum (La III, $Z=57$ ) have been calculated using the relativistic Hartree-Fock (HFR) method. In this method, configuration interaction and relativistic effects have been included in the computations combined with a least squares fitting of the Hamiltonian eigenvalues to the observed energy levels. We have compared the results obtained from this work with the previously available calculations and experiments in literature. We have also reported new transitions with the weighted transition probabilities greater than or equal to $10^{5}$.
\end{abstract}

\section{Introduction}

The radiative properties of the lanthanides and their ions have been rather little considered. This can be explained by the fact that these atoms or ions are characterized by complex electronic structures with an unfilled $4 \mathrm{f}$ subshell, which makes the calculations very difficult, and that the laboratory analyses are still extremely fragmentary or even missing for many ions. Owing to the importance of rare earth elements in astrophysics, especially in relation to nucleosynthesis and star formation (notably the lanthanides in chemically peculiar (CP) stars) [7], there is a growing need for accurate spectroscopic data, that is, wavelengths, radiative transition rates, oscillator strengths, branching fractions, radiative lifetimes, hyperfine structure, and isotope shift data for lanthanide atoms and ions.

The lanthanum atom is the first member of the rare earth elements. Doubly ionized lanthanum (La III) is characterized by a simple atomic structure with core [Xe] and only one outer electron. There is substantial spectroscopic literature concerning La III, though less than the neutral or singly ionized species. The available theoretical and experimental works on energy levels, radiative lifetimes, and transition parameters for La III can be found in the literature $[1-3,5$, $6,8-13]$. These works were reported in our previous work in detail [14].
Up till now the wavelengths, oscillator strengths, and transition probabilities available for La III were obtained by experimental, semiempirical, or pure theoretical approaches. Sixty-five spectral lines of La III in the 2000-12000 $\AA$ interval were reported by Odabasi [2]. Sugar and Kaufman [13] observed forty-five La III spectral lines in the interval from 700 to $2000 \AA$. Johansson and Litzén [5] recorded wavelengths of $5 \mathrm{~d}-4 \mathrm{f}$ lines of La III. Relativistic single-configuration Hartree-Fock oscillator strengths for 6s-6p transitions in La III were reported by Migdalek and Baylis [4]. Migdalek and Wyrozumska [3] have calculated oscillator strengths obtained using the relativistic model-potential approach in there different versions: a model-potential without valencecore electron exchange but with core-polarization included $(\mathrm{RMP}+\mathrm{CP})$, with semiclassical exchange and core-polarization (RMP + SCE + CP), and with empirically adjusted exchange and core-polarization (RMP $+\mathrm{EX}+\mathrm{CP})$ for the $6 \mathrm{~s}-$ 6p, 5d-6p, 5d-4f, 5d-5f, 5d-6f, 6p-6d, and 6p-7d transition arrays. The single-configuration relativistic Hartree-Fock ionization potentials of La III were computed by Migdalek and Bojara [9]. Biémont et al. [1] have performed oscillator strengths and transition probabilities in La III by relativistic Hartree-Fock method with core-polarization.

Our aim here is to determine the transition parameters, such as the wavelengths, oscillator strengths, and transition 
TABLE 1: Wavelengths $\lambda(\AA)$, weighted oscillator strengths $g f$, and weighted transition probabilities $g A_{k i}\left(\mathrm{~s}^{-1}\right)$ for electric dipole $(E 1)$ transitions in La III.

\begin{tabular}{|c|c|c|c|c|c|c|c|}
\hline \multicolumn{2}{|c|}{ Transition } & \multicolumn{2}{|c|}{$\lambda$} & \multicolumn{2}{|c|}{$g f$} & \multicolumn{2}{|c|}{$g A_{k i}$} \\
\hline Lower level & Upper level & This work & Other works & This work & Other works & This work & Other works \\
\hline \multirow{2}{*}{$6 \mathrm{p}^{2} \mathrm{P}_{1 / 2}^{\mathrm{o}}$} & $7 s^{2} S_{1 / 2}$ & $2479.41^{\mathrm{A}, \mathrm{B}}$ & $2478.66^{\mathrm{a}}$ & $0.489^{\mathrm{A}}$ & $0.475^{\mathrm{a}}$ & $5.31 \times 10^{8 \mathrm{~A}}$ & $5.16 \times 10^{8 \mathrm{a}}$ \\
\hline & & & $2478.652^{c}$ & $0.463^{\mathrm{B}}$ & & $5.03 \times 10^{8 \mathrm{~B}}$ & \\
\hline \multirow{2}{*}{$6 \mathrm{p}^{2} \mathrm{P}_{3 / 2}^{\mathrm{o}}$} & $7 s^{2} S_{1 / 2}$ & $2685.55^{\mathrm{A}, \mathrm{B}}$ & $2684.75^{\mathrm{a}}$ & $0.904^{\mathrm{A}}$ & $0.878^{\mathrm{a}}$ & $8.36 \times 10^{8 \mathrm{~A}}$ & $8.12 \times 10^{8 \mathrm{a}}$ \\
\hline & & & $2684.757^{c}$ & $0.855^{\mathrm{B}}$ & & $7.91 \times 10^{8 \mathrm{~B}}$ & \\
\hline \multirow{2}{*}{$6 \mathrm{p}^{2} \mathrm{P}_{1 / 2}^{\mathrm{o}}$} & $6 \mathrm{~d}^{2} \mathrm{D}_{3 / 2}$ & $2477.36^{\mathrm{A}}$ & $2476.60^{\mathrm{a}}$ & $2.474^{\mathrm{A}}$ & $2.365^{\mathrm{a}}$ & $2.69 \times 10^{9 \mathrm{~A}}$ & $2.57 \times 10^{9 \mathrm{a}}$ \\
\hline & & $2477.35^{\mathrm{B}}$ & $2476.599^{c}$ & $2.651^{\mathrm{B}}$ & $2.142^{\mathrm{d}}$ & $2.88 \times 10^{9 \mathrm{~B}}$ & $2.27 \times 10^{9 \mathrm{~b}}$ \\
\hline \multirow[t]{2}{*}{$6 \mathrm{p}^{2} \mathrm{P}_{3 / 2}^{\mathrm{o}}$} & $6 d^{2} D_{5 / 2}$ & $2652.29^{\mathrm{A}}$ & $2651.50^{\mathrm{a}}$ & $4.168^{\mathrm{A}}$ & $3.976^{\mathrm{a}}$ & $3.95 \times 10^{9 \mathrm{~A}}$ & $3.77 \times 10^{9 \mathrm{a}}$ \\
\hline & & $2652.28^{\mathrm{B}}$ & $2651.501^{\mathrm{c}}$ & $4.457^{\mathrm{B}}$ & $3.972^{\mathrm{d}}$ & $4.23 \times 10^{9 \mathrm{~B}}$ & $3.66 \times 10^{9 \mathrm{~b}}$ \\
\hline \multirow[t]{2}{*}{$6 \mathrm{p}^{2} \mathrm{P}_{3 / 2}^{\mathrm{o}}$} & $6 \mathrm{~d}^{2} \mathrm{D}_{3 / 2}$ & $2683.14^{\mathrm{A}}$ & $2682.34^{\mathrm{a}}$ & $0.458^{\mathrm{A}}$ & $0.437^{\mathrm{a}}$ & $4.24 \times 10^{8 \mathrm{~A}}$ & $4.05 \times 10^{8 \mathrm{a}}$ \\
\hline & & $2683.13^{\mathrm{B}}$ & $2682.345^{c}$ & $0.489^{\mathrm{B}}$ & $0.444^{\mathrm{d}}$ & $4.54 \times 10^{8 \mathrm{~B}}$ & $4.01 \times 10^{8 b}$ \\
\hline \multirow{2}{*}{$6 p^{2} P_{1 / 2}^{o}$} & $8 s^{2} S_{1 / 2}$ & $1466.39^{\mathrm{A}, \mathrm{B}}$ & $1466.39^{\mathrm{a}}$ & $0.071^{\mathrm{A}}$ & $0.069^{\mathrm{a}}$ & $2.20 \times 10^{8 \mathrm{~A}}$ & $2.15 \times 10^{8 \mathrm{a}}$ \\
\hline & & & & $0.064^{\mathrm{B}}$ & & $2.00 \times 10^{8 \mathrm{~B}}$ & \\
\hline \multirow[t]{2}{*}{$6 \mathrm{p}^{2} \mathrm{P}_{3 / 2}^{\mathrm{o}}$} & $8 s^{2} S_{1 / 2}$ & $1536.13^{\mathrm{A}, \mathrm{B}}$ & $1536.13^{a}$ & $0.136^{\mathrm{A}}$ & $0.132^{\mathrm{a}}$ & $3.83 \times 10^{8 \mathrm{~A}}$ & $3.73 \times 10^{8 \mathrm{a}}$ \\
\hline & & & & $0.123^{\mathrm{B}}$ & & $3.48 \times 10^{8 \mathrm{~B}}$ & \\
\hline \multirow[t]{2}{*}{$6 \mathrm{p}^{2} \mathrm{P}_{1 / 2}^{\mathrm{o}}$} & $7 d^{2} D_{3 / 2}$ & $1459.45^{\mathrm{A}, \mathrm{B}}$ & $1459.45^{\mathrm{a}}$ & $0.158^{\mathrm{A}}$ & $0.156^{\mathrm{a}}$ & $4.96 \times 10^{8 \mathrm{~A}}$ & $4.88 \times 10^{8 \mathrm{a}}$ \\
\hline & & & & $0.216^{\mathrm{B}}$ & $0.137^{\mathrm{d}}$ & $6.76 \times 10^{8 \mathrm{~B}}$ & $4.26 \times 10^{8 b}$ \\
\hline \multirow{2}{*}{$6 \mathrm{p}^{2} \mathrm{P}_{3 / 2}^{\mathrm{o}}$} & $7 \mathrm{~d}^{2} \mathrm{D}_{5 / 2}$ & $1523.75^{\mathrm{A}, \mathrm{B}}$ & $1523.75^{a}$ & $0.277^{\mathrm{A}}$ & $0.269^{\mathrm{a}}$ & $7.95 \times 10^{8 \mathrm{~A}}$ & $7.71 \times 10^{8 \mathrm{a}}$ \\
\hline & & & & $0.372^{\mathrm{B}}$ & $0.211^{\mathrm{d}}$ & $10.70 \times 10^{8 \mathrm{~B}}$ & $6.02 \times 10^{8 b}$ \\
\hline \multirow[t]{2}{*}{$6 \mathrm{p}^{2} \mathrm{P}_{3 / 2}^{\mathrm{o}}$} & $7 \mathrm{~d}^{2} \mathrm{D}_{3 / 2}$ & $1528.51^{\mathrm{A}, \mathrm{B}}$ & $1528.51^{\mathrm{a}}$ & $0.031^{\mathrm{A}}$ & $0.030^{\mathrm{a}}$ & $8.71 \times 10^{7 \mathrm{~A}}$ & $8.49 \times 10^{7 \mathrm{a}}$ \\
\hline & & & & $0.041^{\mathrm{B}}$ & $0.022^{\mathrm{d}}$ & $11.80 \times 10^{7 \mathrm{~B}}$ & $6.23 \times 10^{7 b}$ \\
\hline \multirow{2}{*}{$6 \mathrm{p}^{2} \mathrm{P}_{1 / 2}^{\mathrm{o}}$} & $9 s^{2} S_{1 / 2}$ & $1212.28^{\mathrm{A}, \mathrm{B}}$ & $1212.28^{\mathrm{a}}$ & $0.026^{\mathrm{A}}$ & $0.026^{\mathrm{a}}$ & $1.19 \times 10^{8 \mathrm{~A}}$ & $1.16 \times 10^{8 \mathrm{a}}$ \\
\hline & & & & $0.023^{\mathrm{B}}$ & & $1.06 \times 10^{8 \mathrm{~B}}$ & \\
\hline \multirow{2}{*}{$6 \mathrm{p}^{2} \mathrm{P}_{3 / 2}^{\mathrm{o}}$} & $9 s^{2} S_{1 / 2}$ & $1259.55^{\mathrm{A}, \mathrm{B}}$ & $1259.55^{\mathrm{a}}$ & $0.051^{\mathrm{A}}$ & $0.049^{\mathrm{a}}$ & $2.13 \times 10^{8 \mathrm{~A}}$ & $2.07 \times 10^{8 \mathrm{a}}$ \\
\hline & & & & $0.045^{\mathrm{B}}$ & & $1.89 \times 10^{8 \mathrm{~B}}$ & \\
\hline \multirow[t]{2}{*}{$6 \mathrm{p}^{2} \mathrm{P}_{1 / 2}^{\mathrm{o}}$} & $8 \mathrm{~d}^{2} \mathrm{D}_{3 / 2}$ & $1208.79^{\mathrm{A}, \mathrm{B}}$ & $1208.79^{a}$ & $0.040^{\mathrm{A}}$ & $0.041^{\mathrm{a}}$ & $1.80 \times 10^{8 \mathrm{~A}}$ & $1.86 \times 10^{8 \mathrm{a}}$ \\
\hline & & & & $0.065^{\mathrm{B}}$ & & $2.95 \times 10^{8 \mathrm{~B}}$ & \\
\hline \multirow[t]{2}{*}{$6 \mathrm{p}^{2} \mathrm{P}_{3 / 2}^{\mathrm{o}}$} & $8 d^{2} D_{5 / 2}$ & $1254.00^{\mathrm{A}, \mathrm{B}}$ & $1254.00^{\mathrm{a}}$ & $0.070^{\mathrm{A}}$ & $0.071^{\mathrm{a}}$ & $2.98 \times 10^{8 \mathrm{~A}}$ & $3.00 \times 10^{8 \mathrm{a}}$ \\
\hline & & & & $0.112^{\mathrm{B}}$ & & $4.76 \times 10^{8 \mathrm{~B}}$ & \\
\hline \multirow{2}{*}{$6 \mathrm{p}^{2} \mathrm{P}_{3 / 2}^{\mathrm{o}}$} & $8 \mathrm{~d}^{2} \mathrm{D}_{3 / 2}$ & $1255.79^{\mathrm{A}, \mathrm{B}}$ & $1255.79^{\mathrm{a}}$ & $0.008^{\mathrm{A}}$ & $0.008^{\mathrm{a}}$ & $3.27 \times 10^{7 \mathrm{~A}}$ & $3.32 \times 10^{7 \mathrm{a}}$ \\
\hline & & & & $0.012^{\mathrm{B}}$ & & $5.27 \times 10^{7 \mathrm{~B}}$ & \\
\hline $6 \mathrm{p}^{2} \mathrm{P}_{1 / 2}^{\mathrm{o}}$ & $10 s^{2} S_{1 / 2}$ & $1101.01^{\mathrm{A}, \mathrm{B}}$ & $1101.01^{\mathrm{a}}$ & $0.013^{\mathrm{A}}$ & $0.013^{\mathrm{a}}$ & $7.26 \times 10^{7 \mathrm{~A}}$ & $7.07 \times 10^{7 \mathrm{a}}$ \\
\hline & & & & $0.012^{\mathrm{B}}$ & & $6.39 \times 10^{7 \mathrm{~B}}$ & \\
\hline $6 \mathrm{p}^{2} \mathrm{P}_{3 / 2}^{\mathrm{o}}$ & $10 s^{2} S_{1 / 2}$ & $1139.87^{\mathrm{A}, \mathrm{B}}$ & $1139.87^{\mathrm{a}}$ & $0.026^{\mathrm{A}}$ & $0.025^{\mathrm{a}}$ & $1.31 \times 10^{8 \mathrm{~A}}$ & $1.27 \times 10^{8 \mathrm{a}}$ \\
\hline & & & & $0.022^{\mathrm{B}}$ & & $1.15 \times 10^{8 \mathrm{~B}}$ & \\
\hline $6 \mathrm{p}^{2} \mathrm{P}_{1 / 2}^{\mathrm{o}}$ & $9 \mathrm{~d}^{2} \mathrm{D}_{3 / 2}$ & $1099.00^{\mathrm{A}, \mathrm{B}}$ & $1099.00^{\mathrm{a}}$ & $0.015^{\mathrm{A}}$ & $0.017^{\mathrm{a}}$ & $8.54 \times 10^{7 \mathrm{~A}}$ & $9.21 \times 10^{7 \mathrm{a}}$ \\
\hline & & & & $0.029^{\mathrm{B}}$ & & $16.10 \times 10^{7 \mathrm{~B}}$ & \\
\hline $6 \mathrm{p}^{2} \mathrm{P}_{3 / 2}^{\mathrm{o}}$ & $9 \mathrm{~d}^{2} \mathrm{D}_{5 / 2}$ & $1136.80^{\mathrm{A}, \mathrm{B}}$ & $1136.80^{\mathrm{a}}$ & $0.028^{\mathrm{A}}$ & $0.029^{\mathrm{a}}$ & $1.44 \times 10^{8 \mathrm{~A}}$ & $1.50 \times 10^{8 \mathrm{a}}$ \\
\hline & & & & $0.050^{\mathrm{B}}$ & & $2.61 \times 10^{8 \mathrm{~B}}$ & \\
\hline $6 \mathrm{p}^{2} \mathrm{P}_{3 / 2}^{\mathrm{o}}$ & $9 \mathrm{~d}^{2} \mathrm{D}_{3 / 2}$ & $1137.71^{\mathrm{A}, \mathrm{B}}$ & $1137.71^{\mathrm{a}}$ & $0.003^{\mathrm{A}}$ & $0.003^{\mathrm{a}}$ & $1.58 \times 10^{7 \mathrm{~A}}$ & $1.66 \times 10^{7 \mathrm{a}}$ \\
\hline & & & & $0.006^{\mathrm{B}}$ & & $2.89 \times 10^{7 \mathrm{~B}}$ & \\
\hline $6 d^{2} D_{3 / 2}$ & $5 f^{2} F_{5 / 2}^{o}$ & $9926.70^{\mathrm{A}}$ & $9924.04^{\mathrm{a}}$ & $2.549^{\mathrm{A}}$ & $2.370^{\mathrm{a}}$ & $1.73 \times 10^{8 \mathrm{~A}}$ & $1.60 \times 10^{8 \mathrm{a}}$ \\
\hline & & $9926.74^{\mathrm{B}}$ & $9923.989^{c}$ & $2.574^{\mathrm{B}}$ & & $1.74 \times 10^{8 \mathrm{~B}}$ & \\
\hline $6 \mathrm{~d}^{2} \mathrm{D}_{5 / 2}$ & $5 \mathrm{f}^{2} \mathrm{~F}_{7 / 2}^{\mathrm{o}}$ & $10287.59^{\mathrm{A}, \mathrm{B}}$ & $10284.790^{c}$ & $3.515^{\mathrm{A}}$ & - & $2.21 \times 10^{8 \mathrm{~A}}$ & - \\
\hline & & & & $3.548^{\mathrm{B}}$ & & $2.24 \times 10^{8 \mathrm{~B}}$ & \\
\hline $6 \mathrm{~d}^{2} \mathrm{D}_{5 / 2}$ & $5 \mathrm{f}^{2} \mathrm{~F}_{5 / 2}^{\mathrm{o}}$ & $10373.15^{\mathrm{A}}$ & $10370.335^{\mathrm{c}}$ & $0.174^{\mathrm{A}}$ & - & $1.08 \times 10^{7 \mathrm{~A}}$ & - \\
\hline & & $10373.12^{\mathrm{B}}$ & & $0.176^{\mathrm{B}}$ & & $1.09 \times 10^{7 \mathrm{~B}}$ & \\
\hline $6 \mathrm{~d}^{2} \mathrm{D}_{3 / 2}$ & $7 \mathrm{p}^{2} \mathrm{P}_{3 / 2}^{\mathrm{o}}$ & $8277.67^{\mathrm{A}, \mathrm{B}}$ & $8275.41^{\mathrm{a}}$ & $0.243^{\mathrm{A}}$ & $0.240^{\mathrm{a}}$ & $2.37 \times 10^{7 \mathrm{~A}}$ & $2.34 \times 10^{7 \mathrm{a}}$ \\
\hline & & & $8275.388^{c}$ & $0.250^{\mathrm{B}}$ & & $2.43 \times 10^{7 \mathrm{~B}}$ & \\
\hline $6 d^{2} D_{5 / 2}$ & $7 \mathrm{p}^{2} \mathrm{P}_{3 / 2}^{\mathrm{o}}$ & $8585.81^{\mathrm{A}}$ & $8583.42^{\mathrm{a}}$ & $2.115^{\mathrm{A}}$ & $2.081^{\mathrm{a}}$ & $1.91 \times 10^{8 \mathrm{~A}}$ & $1.88 \times 10^{8 \mathrm{a}}$ \\
\hline & & $8585.76^{\mathrm{B}}$ & $8583.453^{c}$ & $2.165^{\mathrm{B}}$ & & $1.96 \times 10^{8 \mathrm{~B}}$ & \\
\hline
\end{tabular}


TABle 1: Continued.

\begin{tabular}{|c|c|c|c|c|c|c|c|}
\hline \multicolumn{2}{|c|}{ Transition } & \multicolumn{2}{|c|}{$\lambda$} & \multicolumn{2}{|c|}{$g f$} & \multicolumn{2}{|c|}{$g A_{k i}$} \\
\hline Lower level & Upper level & This work & Other works & This work & Other works & This work & Other works \\
\hline \multirow[t]{2}{*}{$6 \mathrm{~d}^{2} \mathrm{D}_{3 / 2}$} & $7 \mathrm{p}^{2} \mathrm{P}_{1 / 2}^{\mathrm{o}}$ & $9215.20^{\mathrm{A}}$ & $9212.68^{\mathrm{a}}$ & $1.094^{\mathrm{A}}$ & $1.077^{\mathrm{a}}$ & $8.59 \times 10^{7 \mathrm{~A}}$ & $8.46 \times 10^{7 a}$ \\
\hline & & $9215.23^{\mathrm{B}}$ & $9212.628^{c}$ & $1.121^{\mathrm{B}}$ & & $8.80 \times 10^{7 \mathrm{~B}}$ & \\
\hline \multirow[t]{2}{*}{$6 d^{2} D_{3 / 2}$} & $6 \mathrm{f}^{2} \mathrm{~F}_{5 / 2}^{\mathrm{o}}$ & $3076.05^{\mathrm{A}}$ & $3075.17^{\mathrm{a}}$ & $0.733^{\mathrm{A}}$ & $0.822^{\mathrm{a}}$ & $5.17 \times 10^{8 \mathrm{~A}}$ & $5.80 \times 10^{8 \mathrm{a}}$ \\
\hline & & $3076.06^{\mathrm{B}}$ & $3075.173^{c}$ & $0.790^{\mathrm{B}}$ & & $5.57 \times 10^{8 \mathrm{~B}}$ & \\
\hline \multirow{2}{*}{$6 d^{2} D_{5 / 2}$} & $6 f^{2} \mathrm{~F}_{7 / 2}^{\mathrm{o}}$ & $3112.88^{\mathrm{A}, \mathrm{B}}$ & $3111.97^{\mathrm{a}}$ & $1.037^{\mathrm{A}}$ & $1.161^{\mathrm{a}}$ & $7.14 \times 10^{8 \mathrm{~A}}$ & $7.99 \times 10^{8 \mathrm{a}}$ \\
\hline & & & $3111.969^{c}$ & $1.116^{\mathrm{B}}$ & & $7.68 \times 10^{8 \mathrm{~B}}$ & \\
\hline \multirow[t]{2}{*}{$6 d^{2} D_{5 / 2}$} & $6 f^{2} F_{5 / 2}^{o}$ & $3117.63^{\mathrm{A}, \mathrm{B}}$ & $3116.74^{\mathrm{a}}$ & $0.052^{\mathrm{A}}$ & $0.058^{\mathrm{a}}$ & $3.55 \times 10^{7 \mathrm{~A}}$ & $3.98 \times 10^{7 \mathrm{a}}$ \\
\hline & & & $3116.738^{c}$ & $0.056^{\mathrm{B}}$ & & $3.82 \times 10^{7 \mathrm{~B}}$ & \\
\hline \multirow[t]{2}{*}{$6 \mathrm{~d}^{2} \mathrm{D}_{3 / 2}$} & $8 \mathrm{p}^{2} \mathrm{P}_{3 / 2}^{\mathrm{o}}$ & $2954.63^{\mathrm{A}}$ & $2953.77^{\mathrm{a}}$ & $0.009^{\mathrm{A}}$ & $0.008^{\mathrm{a}}$ & $6.82 \times 10^{6 \mathrm{~A}}$ & $5.87 \times 10^{6 a}$ \\
\hline & & $2954.64^{\mathrm{B}}$ & & $0.008^{\mathrm{B}}$ & & $6.18 \times 10^{6 \mathrm{~B}}$ & \\
\hline \multirow{2}{*}{$6 d^{2} D_{5 / 2}$} & $8 \mathrm{p}^{2} \mathrm{P}_{3 / 2}^{\mathrm{o}}$ & $2992.97^{\mathrm{A}, \mathrm{B}}$ & $2992.10^{\mathrm{a}}$ & $0.079^{\mathrm{A}}$ & $0.068^{\mathrm{a}}$ & $5.89 \times 10^{7 \mathrm{~A}}$ & $5.08 \times 10^{7 \mathrm{a}}$ \\
\hline & & & $2992.098^{c}$ & $0.072^{\mathrm{B}}$ & & $5.35 \times 10^{7 \mathrm{~B}}$ & \\
\hline \multirow[t]{2}{*}{$6 d^{2} D_{3 / 2}$} & $8 \mathrm{p}^{2} \mathrm{P}_{1 / 2}^{\mathrm{o}}$ & $3010.10^{\mathrm{A}}$ & $3009.22^{\mathrm{a}}$ & $0.044^{\mathrm{A}}$ & $0.038^{\mathrm{a}}$ & $3.23 \times 10^{7 \mathrm{~A}}$ & $2.77 \times 10^{7 a}$ \\
\hline & & $3010.11^{\mathrm{B}}$ & $3009.223^{c}$ & $0.040^{\mathrm{B}}$ & & $2.92 \times 10^{7 \mathrm{~B}}$ & \\
\hline \multirow[t]{2}{*}{$6 \mathrm{~d}^{2} \mathrm{D}_{3 / 2}$} & $7 \mathrm{f}^{2} \mathrm{~F}_{5 / 2}^{\mathrm{o}}$ & $2239.04^{\mathrm{A}}$ & $2238.35^{\mathrm{a}}$ & $0.339^{\mathrm{A}}$ & $0.358^{\mathrm{a}}$ & $4.51 \times 10^{8 \mathrm{~A}}$ & $4.76 \times 10^{8 \mathrm{a}}$ \\
\hline & & $2239.05^{\mathrm{B}}$ & & $0.356^{\mathrm{B}}$ & & $4.74 \times 10^{8 \mathrm{~B}}$ & \\
\hline \multirow{2}{*}{$6 \mathrm{~d}^{2} \mathrm{D}_{5 / 2}$} & $7 \mathrm{f}^{2} \mathrm{~F}_{7 / 2}^{\mathrm{o}}$ & $2259.31^{\mathrm{A}, \mathrm{B}}$ & $2258.61^{\mathrm{a}}$ & $0.480^{\mathrm{A}}$ & $0.507^{\mathrm{a}}$ & $6.28 \times 10^{8 \mathrm{~A}}$ & $6.62 \times 10^{8 \mathrm{a}}$ \\
\hline & & & $2258.609^{c}$ & $0.504^{\mathrm{B}}$ & & $6.59 \times 10^{8 \mathrm{~B}}$ & \\
\hline \multirow[t]{2}{*}{$6 d^{2} D_{5 / 2}$} & $7 f^{2} F_{5 / 2}^{o}$ & $2261.00^{\mathrm{A}, \mathrm{B}}$ & $2260.30^{\mathrm{a}}$ & $0.024^{\mathrm{A}}$ & $0.025^{\mathrm{a}}$ & $3.13 \times 10^{7 \mathrm{~A}}$ & $3.30 \times 10^{7 \mathrm{a}}$ \\
\hline & & & $2260.295^{c}$ & $0.025^{\mathrm{B}}$ & & $3.29 \times 10^{7 \mathrm{~B}}$ & \\
\hline \multirow[t]{2}{*}{$6 \mathrm{~d}^{2} \mathrm{D}_{3 / 2}$} & $9 \mathrm{p}^{2} \mathrm{P}_{3 / 2}^{\mathrm{o}}$ & $2195.18^{\mathrm{A}}$ & $2194.50^{\mathrm{a}}$ & $0.003^{\mathrm{A}, \mathrm{B}}$ & $0.002^{\mathrm{a}}$ & $4.15 \times 10^{6 \mathrm{~A}}$ & $3.28 \times 10^{6 \mathrm{a}}$ \\
\hline & & $2195.19^{\mathrm{B}}$ & & & & $3.58 \times 10^{6 \mathrm{~B}}$ & \\
\hline \multirow{2}{*}{$6 \mathrm{~d}^{2} \mathrm{D}_{3 / 2}$} & $9 \mathrm{p}^{2} \mathrm{P}_{1 / 2}^{\mathrm{o}}$ & $2213.95^{\mathrm{A}, \mathrm{B}}$ & $2213.26^{a}$ & $0.015^{\mathrm{A}}$ & $0.012^{\mathrm{a}}$ & $2.02 \times 10^{7 \mathrm{~A}}$ & $1.60 \times 10^{7 \mathrm{a}}$ \\
\hline & & & & $0.013^{\mathrm{B}}$ & & $1.75 \times 10^{7 \mathrm{~B}}$ & \\
\hline \multirow[t]{2}{*}{$6 d^{2} D_{5 / 2}$} & $9 \mathrm{p}^{2} \mathrm{P}_{3 / 2}^{\mathrm{o}}$ & $2216.28^{\mathrm{A}, \mathrm{B}}$ & $2215.58^{a}$ & $0.027^{\mathrm{A}}$ & $0.021^{\mathrm{a}}$ & $3.62 \times 10^{7 \mathrm{~A}}$ & $2.87 \times 10^{7 a}$ \\
\hline & & & & $0.023^{\mathrm{B}}$ & & $3.13 \times 10^{7 \mathrm{~B}}$ & \\
\hline \multirow{2}{*}{$6 \mathrm{~d}^{2} \mathrm{D}_{3 / 2}$} & $8 \mathrm{f}^{2} \mathrm{~F}_{5 / 2}^{\mathrm{o}}$ & $1923.33^{\mathrm{A}}$ & $1923.33^{\mathrm{a}}$ & $0.178^{\mathrm{A}}$ & $0.185^{\mathrm{a}}$ & $3.22 \times 10^{8 \mathrm{~A}}$ & $3.33 \times 10^{8 \mathrm{a}}$ \\
\hline & & $1923.34^{\mathrm{B}}$ & & $0.188^{\mathrm{B}}$ & & $3.39 \times 10^{8 \mathrm{~B}}$ & \\
\hline \multirow[t]{2}{*}{$6 \mathrm{~d}^{2} \mathrm{D}_{5 / 2}$} & $8 \mathrm{f}^{2} \mathrm{~F}_{7 / 2}^{\mathrm{o}}$ & $1938.53^{\mathrm{A}, \mathrm{B}}$ & $1938.53^{\mathrm{a}}$ & $0.253^{\mathrm{A}}$ & $0.262^{\mathrm{a}}$ & $4.49 \times 10^{8 \mathrm{~A}}$ & $4.65 \times 10^{8 \mathrm{a}}$ \\
\hline & & & & $0.267^{\mathrm{B}}$ & & $4.73 \times 10^{8 \mathrm{~B}}$ & \\
\hline $6 d^{2} D_{5 / 2}$ & $8 \mathrm{f}^{2} \mathrm{~F}_{5 / 2}^{\mathrm{o}}$ & $1939.51^{\mathrm{A}, \mathrm{B}}$ & $1939.51^{\mathrm{a}}$ & $0.013^{\mathrm{A}, \mathrm{B}}$ & $0.013^{\mathrm{a}}$ & $2.24 \times 10^{7 \mathrm{~A}}$ & $2.32 \times 10^{7 \mathrm{a}}$ \\
\hline & & & & & & $2.36 \times 10^{7 \mathrm{~B}}$ & \\
\hline $6 f^{2} F_{5 / 2}^{o}$ & $6 \mathrm{~g}^{2} \mathrm{G}_{7 / 2}$ & $8290.18^{\mathrm{A}}$ & $8287.76^{\mathrm{a}}$ & $8.903^{\mathrm{A}}$ & $8.527^{a}$ & $8.64 \times 10^{8 \mathrm{~A}, \mathrm{~B}}$ & $8.28 \times 10^{8 \mathrm{a}}$ \\
\hline & & $8290.16^{\mathrm{B}}$ & $8287.752^{c}$ & $8.904^{\mathrm{B}}$ & & & \\
\hline $6 \mathrm{f}^{2} \mathrm{~F}_{7 / 2}^{\mathrm{o}}$ & $6 \mathrm{~g}^{2} \mathrm{G}_{9 / 2}$ & $8323.43^{\mathrm{A}}$ & $8321.16^{\mathrm{a}}$ & $11.495^{\mathrm{A}}$ & $11.009^{\mathrm{a}}$ & $1.11 \times 10^{9 \mathrm{~A}, \mathrm{~B}}$ & $1.06 \times 10^{9 a}$ \\
\hline & & $8323.34^{\mathrm{B}}$ & $8321.107^{c}$ & $11.496^{\mathrm{B}}$ & & & \\
\hline $6 \mathrm{f}^{2} \mathrm{~F}_{7 / 2}^{\mathrm{o}}$ & $6 \mathrm{~g}^{2} \mathrm{G}_{7 / 2}$ & $8323.98^{\mathrm{A}}$ & $8321.63^{\mathrm{a}}$ & $0.328^{\mathrm{A}, \mathrm{B}}$ & $0.315^{\mathrm{a}}$ & $3.16 \times 10^{7 \mathrm{~A}, \mathrm{~B}}$ & $3.03 \times 10^{7 \mathrm{a}}$ \\
\hline & & $8323.97^{\mathrm{B}}$ & & & & & \\
\hline $6 f^{2} F_{5 / 2}^{o}$ & $7 \mathrm{~g}^{2} \mathrm{G}_{7 / 2}$ & $5147.17^{\mathrm{A}, \mathrm{B}}$ & $5145.72^{\mathrm{a}}$ & $1.282^{\mathrm{A}}$ & $1.239^{\mathrm{a}}$ & $3.23 \times 10^{8 \mathrm{~A}, \mathrm{~B}}$ & $3.12 \times 10^{8 \mathrm{a}}$ \\
\hline & & & $5145.729^{c}$ & $1.283^{\mathrm{B}}$ & & & \\
\hline $6 \mathrm{f}^{2} \mathrm{~F}_{7 / 2}^{\mathrm{o}}$ & $7 \mathrm{~g}^{2} \mathrm{G}_{9 / 2}$ & $5159.84^{\mathrm{A}}$ & $5158.39^{\mathrm{a}}$ & $1.658^{\mathrm{A}}$ & $1.602^{\mathrm{a}}$ & $4.15 \times 10^{8 \mathrm{~A}}$ & $4.01 \times 10^{8 \mathrm{a}}$ \\
\hline & & $5159.82^{\mathrm{B}}$ & $5158.410^{c}$ & $1.659^{\mathrm{B}}$ & & $4.16 \times 10^{8 \mathrm{~B}}$ & \\
\hline $6 \mathrm{f}^{2} \mathrm{~F}_{7 / 2}^{\mathrm{o}}$ & $7 \mathrm{~g}^{2} \mathrm{G}_{7 / 2}$ & $5160.18^{\mathrm{A}}$ & $5158.76^{\mathrm{a}}$ & $0.047^{\mathrm{A}, \mathrm{B}}$ & $0.046^{\mathrm{a}}$ & $1.19 \times 10^{7 \mathrm{~A}, \mathrm{~B}}$ & $1.15 \times 10^{7 \mathrm{a}}$ \\
\hline & & $5160.19^{\mathrm{B}}$ & & & & & \\
\hline $6 f^{2} F_{5 / 2}^{o}$ & $8 \mathrm{~g}^{2} \mathrm{G}_{7 / 2}$ & $4130.43^{\mathrm{A}, \mathrm{B}}$ & $4129.24^{\mathrm{a}}$ & $0.411^{\mathrm{A}, \mathrm{B}}$ & $0.394^{\mathrm{a}}$ & $1.61 \times 10^{8 \mathrm{~A}, \mathrm{~B}}$ & $1.54 \times 10^{8 \mathrm{a}}$ \\
\hline $6 \mathrm{f}^{2} \mathrm{~F}_{7 / 2}^{\mathrm{o}}$ & $8 g^{2} \mathrm{G}_{9 / 2}$ & $4138.59^{\mathrm{A}}$ & $4137.43^{\mathrm{a}}$ & $0.531^{\mathrm{A}}$ & $0.509^{\mathrm{a}}$ & $2.07 \times 10^{8 \mathrm{~A}, \mathrm{~B}}$ & $1.98 \times 10^{8 \mathrm{a}}$ \\
\hline & & $4138.58^{\mathrm{B}}$ & $4137.428^{c}$ & $0.532^{\mathrm{B}}$ & & & \\
\hline $6 \mathrm{f}^{2} \mathrm{~F}_{7 / 2}^{\mathrm{o}}$ & $8 \mathrm{~g}^{2} \mathrm{G}_{7 / 2}$ & $4138.80^{\mathrm{A}}$ & $4137.64^{\mathrm{a}}$ & $0.015^{\mathrm{A}, \mathrm{B}}$ & $0.015^{\mathrm{a}}$ & $5.91 \times 10^{6 \mathrm{~A}, \mathrm{~B}}$ & $5.67 \times 10^{6 a}$ \\
\hline & & $4138.81^{\mathrm{B}}$ & & & & & \\
\hline $6 f^{2} F_{5 / 2}^{o}$ & $9 d^{2} D_{3 / 2}$ & $5519.77^{\mathrm{A}}$ & $5518.19^{a}$ & $0.132^{\mathrm{A}}$ & $0.130^{\mathrm{a}}$ & $2.89 \times 10^{7 \mathrm{~A}}$ & $2.85 \times 10^{7 \mathrm{a}}$ \\
\hline & & $5519.75^{\text {B }}$ & $5518.187^{c}$ & $0.128^{\mathrm{B}}$ & & $2.79 \times 10^{7 \mathrm{~B}}$ & \\
\hline
\end{tabular}


TABle 1: Continued.

\begin{tabular}{|c|c|c|c|c|c|c|c|}
\hline \multicolumn{2}{|c|}{ Transition } & \multicolumn{2}{|c|}{$\lambda$} & \multicolumn{2}{|c|}{$g f$} & \multicolumn{2}{|c|}{$g A_{k i}$} \\
\hline Lower level & Upper level & This work & Other works & This work & Other works & This work & Other works \\
\hline \multirow[t]{2}{*}{$6 f^{2} F_{5 / 2}^{\circ}$} & $9 \mathrm{~d}^{2} \mathrm{D}_{5 / 2}$ & $5498.45^{\mathrm{A}}$ & $5496.88^{\mathrm{a}}$ & $0.009^{\mathrm{A}, \mathrm{B}}$ & $0.009^{\mathrm{a}}$ & $2.08 \times 10^{6 \mathrm{~A}}$ & $2.06 \times 10^{6 a}$ \\
\hline & & $5498.43^{\mathrm{B}}$ & & & & $2.02 \times 10^{6 \mathrm{~B}}$ & \\
\hline \multirow{2}{*}{$6 \mathrm{f}^{2} \mathrm{~F}_{7 / 2}^{\mathrm{o}}$} & $9 \mathrm{~d}^{2} \mathrm{D}_{5 / 2}$ & $5513.29^{\mathrm{A}, \mathrm{B}}$ & $5511.76^{\mathrm{a}}$ & $0.188^{\mathrm{A}}$ & $0.186^{\mathrm{a}}$ & $4.14 \times 10^{7 \mathrm{~A}}$ & $4.09 \times 10^{7 \mathrm{a}}$ \\
\hline & & & $5511.721^{\mathrm{c}}$ & $0.182^{\mathrm{B}}$ & & $4.01 \times 10^{7 \mathrm{~B}}$ & \\
\hline \multirow[t]{3}{*}{$6 s^{2} S_{1 / 2}$} & $6 \mathrm{p}^{2} \mathrm{P}_{3 / 2}^{\mathrm{o}}$ & $3172.60^{\mathrm{A}, \mathrm{B}}$ & $3171.69^{a}$ & $1.673^{\mathrm{A}}$ & $1.527^{\mathrm{a}}$ & $1.11 \times 10^{9 \mathrm{~A}}$ & $1.01 \times 10^{9 \mathrm{a}}$ \\
\hline & & & $3171.735^{c}$ & $1.935^{\mathrm{B}}$ & $1.418^{\mathrm{d}}$ & $1.28 \times 10^{9 \mathrm{~B}}$ & $9.40 \times 10^{8 b}$ \\
\hline & & & & & $1.868^{\mathrm{e}}$ & & \\
\hline \multirow[t]{3}{*}{$6 s^{2} S_{1 / 2}$} & $6 \mathrm{p}^{2} \mathrm{P}_{1 / 2}^{\mathrm{o}}$ & $3518.16^{\mathrm{A}}$ & $3517.16^{\mathrm{a}}$ & $0.754^{\mathrm{A}}$ & $0.689^{a}$ & $4.06 \times 10^{8 \mathrm{~A}}$ & $3.71 \times 10^{8 \mathrm{a}}$ \\
\hline & & $3518.15^{\mathrm{B}}$ & $3517.217^{c}$ & $0.872^{\mathrm{B}}$ & $0.640^{\mathrm{d}}$ & $4.70 \times 10^{8 \mathrm{~B}}$ & $3.45 \times 10^{8 b}$ \\
\hline & & & & & $0.850^{\mathrm{e}}$ & & \\
\hline \multirow{2}{*}{$6 s^{2} S_{1 / 2}$} & $7 \mathrm{p}^{2} \mathrm{P}_{3 / 2}^{\mathrm{o}}$ & $1236.55^{\mathrm{A}, \mathrm{B}}$ & $1236.55^{\mathrm{a}}$ & $0.002^{\mathrm{A}}$ & $0.006^{\mathrm{a}}$ & $0.70 \times 10^{7 \mathrm{~A}}$ & $2.39 \times 10^{7 \mathrm{a}}$ \\
\hline & & & & $0.001^{\mathrm{B}}$ & & $0.27 \times 10^{7 \mathrm{~B}}$ & \\
\hline \multirow[t]{2}{*}{$6 s^{2} S_{1 / 2}$} & $7 \mathrm{p}^{2} \mathrm{P}_{1 / 2}^{\mathrm{o}}$ & $1255.63^{\mathrm{A}, \mathrm{B}}$ & $1255.63^{\mathrm{a}}$ & $0.001^{\mathrm{A}}$ & $0.003^{\mathrm{a}}$ & $0.33 \times 10^{7 \mathrm{~A}}$ & $1.14 \times 10^{7 \mathrm{a}}$ \\
\hline & & & & $0.0003^{\mathrm{B}}$ & & $0.13 \times 10^{7 \mathrm{~B}}$ & \\
\hline \multirow{2}{*}{$7 \mathrm{~s}^{2} \mathrm{~S}_{1 / 2}$} & $7 \mathrm{p}^{2} \mathrm{P}_{3 / 2}^{\mathrm{o}}$ & $8254.85^{\mathrm{A}}$ & $8252.53^{\mathrm{a}}$ & $2.418^{\mathrm{A}}$ & $2.279^{\mathrm{a}}$ & $2.37 \times 10^{8 \mathrm{~A}, \mathrm{~B}}$ & $2.23 \times 10^{8 \mathrm{a}}$ \\
\hline & & $8254.77^{\mathrm{B}}$ & $8252.603^{c}$ & $2.424^{\mathrm{B}}$ & & & \\
\hline \multirow[t]{2}{*}{$7 \mathrm{~s}^{2} \mathrm{~S}_{1 / 2}$} & $7 \mathrm{p}^{2} \mathrm{P}_{1 / 2}^{\mathrm{o}}$ & $9186.87^{\mathrm{A}}$ & $9184.34^{\mathrm{a}}$ & $1.086^{\mathrm{A}}$ & $1.024^{\mathrm{a}}$ & $8.59 \times 10^{7 \mathrm{~A}}$ & $8.09 \times 10^{7 \mathrm{a}}$ \\
\hline & & $9186.92^{\mathrm{B}}$ & $9184.380^{c}$ & $1.089^{\mathrm{B}}$ & & $8.61 \times 10^{7 \mathrm{~B}}$ & \\
\hline \multirow[t]{2}{*}{$7 \mathrm{~s}^{2} \mathrm{~S}_{1 / 2}$} & $8 \mathrm{p}^{2} \mathrm{P}_{3 / 2}^{\mathrm{o}}$ & $2951.72^{\mathrm{A}, \mathrm{B}}$ & $2950.843^{c}$ & $0.002^{\mathrm{A}, \mathrm{B}}$ & - & $1.81 \times 10^{6 \mathrm{~A}}$ & - \\
\hline & & & & & & $1.54 \times 10^{6 \mathrm{~B}}$ & \\
\hline \multirow[t]{2}{*}{$7 s^{2} S_{1 / 2}$} & $8 \mathrm{p}^{2} \mathrm{P}_{1 / 2}^{\mathrm{o}}$ & $3007.07^{\mathrm{A}}$ & $3006.186^{c}$ & $0.001^{\mathrm{A}, \mathrm{B}}$ & - & $8.57 \times 10^{5 \mathrm{~A}}$ & - \\
\hline & & $3007.08^{\mathrm{B}}$ & & & & $7.27 \times 10^{5 \mathrm{~B}}$ & \\
\hline \multirow[t]{2}{*}{$7 \mathrm{p}^{2} \mathrm{P}_{1 / 2}^{\mathrm{o}}$} & $8 s^{2} S_{1 / 2}$ & $5890.23^{\mathrm{A}}$ & $5888.63^{\mathrm{a}}$ & $0.716^{\mathrm{A}}$ & $0.718^{\mathrm{a}}$ & $1.38 \times 10^{8 \mathrm{~A}}$ & $1.38 \times 10^{8 \mathrm{a}}$ \\
\hline & & $5890.25^{\mathrm{B}}$ & $5888.620^{c}$ & $0.714^{\mathrm{B}}$ & & $1.37 \times 10^{8 \mathrm{~B}}$ & \\
\hline \multirow{2}{*}{$7 \mathrm{p}^{2} \mathrm{P}_{3 / 2}^{\mathrm{o}}$} & $8 s^{2} S_{1 / 2}$ & $6349.93^{\mathrm{A}}$ & $6348.21^{\mathrm{a}}$ & $1.329^{\mathrm{A}}$ & $1.331^{\mathrm{a}}$ & $2.20 \times 10^{8 \mathrm{~A}}$ & $2.20 \times 10^{8 \mathrm{a}}$ \\
\hline & & $6349.97^{\mathrm{B}}$ & $6348.213^{c}$ & $1.324^{\mathrm{B}}$ & & $2.19 \times 10^{8 \mathrm{~B}}$ & \\
\hline \multirow[t]{2}{*}{$7 \mathrm{p}^{2} \mathrm{P}_{1 / 2}^{\mathrm{o}}$} & $7 d^{2} D_{3 / 2}$ & $5779.74^{\mathrm{A}}$ & $5778.14^{\mathrm{a}}$ & $3.095^{\mathrm{A}}$ & $2.967^{\mathrm{a}}$ & $6.18 \times 10^{8 \mathrm{~A}}$ & $5.92 \times 10^{8 \mathrm{a}}$ \\
\hline & & $5779.71^{\mathrm{B}}$ & $5778.138^{c}$ & $3.045^{\mathrm{B}}$ & & $6.08 \times 10^{8 \mathrm{~B}}$ & \\
\hline \multirow{2}{*}{$7 \mathrm{p}^{2} \mathrm{P}_{3 / 2}^{\mathrm{o}}$} & $7 d^{2} D_{5 / 2}$ & $6143.64^{\mathrm{A}}$ & $6141.99^{a}$ & $5.238^{\mathrm{A}}$ & $5.024^{\mathrm{a}}$ & $9.26 \times 10^{8 \mathrm{~A}}$ & $8.88 \times 10^{8 a}$ \\
\hline & & $6143.71^{\mathrm{B}}$ & $6141.987^{c}$ & $5.157^{\mathrm{B}}$ & & $9.11 \times 10^{8 \mathrm{~B}}$ & \\
\hline $7 \mathrm{p}^{2} \mathrm{P}_{3 / 2}^{\mathrm{o}}$ & $7 d^{2} D_{3 / 2}$ & $6221.70^{\mathrm{A}}$ & $6219.99^{a}$ & $0.575^{\mathrm{A}}$ & $0.551^{\mathrm{a}}$ & $9.91 \times 10^{7 \mathrm{~A}}$ & $9.50 \times 10^{7 \mathrm{a}}$ \\
\hline & & $6221.69^{\mathrm{B}}$ & $6219.999^{c}$ & $0.566^{\mathrm{B}}$ & & $9.75 \times 10^{7 \mathrm{~B}}$ & \\
\hline $7 \mathrm{p}^{2} \mathrm{P}_{1 / 2}^{\mathrm{o}}$ & $9 \mathrm{~s}^{2} \mathrm{~S}_{1 / 2}$ & $3197.77^{\mathrm{A}}$ & $3196.85^{\mathrm{a}}$ & $0.089^{\mathrm{A}, \mathrm{B}}$ & $0.092^{\mathrm{a}}$ & $5.77 \times 10^{7 \mathrm{~A}, \mathrm{~B}}$ & $5.98 \times 10^{7 \mathrm{a}}$ \\
\hline & & $3197.78^{\mathrm{B}}$ & $3196.844^{c}$ & & & & \\
\hline $7 \mathrm{p}^{2} \mathrm{P}_{3 / 2}^{\mathrm{o}}$ & $9 s^{2} S_{1 / 2}$ & $3328.60^{\mathrm{A}}$ & $3327.64^{\mathrm{a}}$ & $0.170^{\mathrm{A}, \mathrm{B}}$ & $0.176^{\mathrm{a}}$ & $1.02 \times 10^{8 \mathrm{~A}, \mathrm{~B}}$ & $1.06 \times 10^{8 \mathrm{a}}$ \\
\hline & & $3328.61^{\mathrm{B}}$ & $3327.655^{c}$ & & & & \\
\hline $7 \mathrm{p}^{2} \mathrm{P}_{1 / 2}^{\mathrm{o}}$ & $8 \mathrm{~d}^{2} \mathrm{D}_{3 / 2}$ & $3173.60^{\mathrm{A}}$ & $3172.69^{\mathrm{a}}$ & $0.274^{\mathrm{A}}$ & $0.260^{\mathrm{a}}$ & $1.81 \times 10^{8 \mathrm{~A}}$ & $1.72 \times 10^{8 \mathrm{a}}$ \\
\hline & & $3173.61^{\mathrm{B}}$ & $3172.689^{c}$ & $0.284^{\mathrm{B}}$ & & $1.89 \times 10^{8 \mathrm{~B}}$ & \\
\hline $7 \mathrm{p}^{2} \mathrm{P}_{3 / 2}^{\mathrm{o}}$ & $8 \mathrm{~d}^{2} \mathrm{D}_{5 / 2}$ & $3290.05^{\mathrm{A}}$ & $3289.11^{\mathrm{a}}$ & $0.476^{\mathrm{A}}$ & $0.452^{\mathrm{a}}$ & $2.94 \times 10^{8 \mathrm{~A}}$ & $2.79 \times 10^{8 \mathrm{a}}$ \\
\hline & & $3290.07^{\mathrm{B}}$ & $3289.110^{c}$ & $0.494^{\mathrm{B}}$ & & $3.05 \times 10^{8 \mathrm{~B}}$ & \\
\hline $7 \mathrm{p}^{2} \mathrm{P}_{3 / 2}^{\mathrm{o}}$ & $8 \mathrm{~d}^{2} \mathrm{D}_{3 / 2}$ & $3302.41^{\mathrm{A}}$ & $3301.47^{\mathrm{a}}$ & $0.053^{\mathrm{A}}$ & $0.050^{\mathrm{a}}$ & $3.22 \times 10^{7 \mathrm{~A}}$ & $3.06 \times 10^{7 \mathrm{a}}$ \\
\hline & & $3302.43^{\mathrm{B}}$ & $3301.481^{c}$ & $0.055^{\mathrm{B}}$ & & $3.35 \times 10^{7 \mathrm{~B}}$ & \\
\hline $7 \mathrm{p}^{2} \mathrm{P}_{1 / 2}^{\mathrm{o}}$ & $10 s^{2} S_{1 / 2}$ & $2524.74^{\mathrm{A}, \mathrm{B}}$ & $2523.98^{\mathrm{a}}$ & $0.031^{\mathrm{A}, \mathrm{B}}$ & $0.033^{\mathrm{a}}$ & $3.25 \times 10^{7 \mathrm{~A}}$ & $3.44 \times 10^{7 \mathrm{a}}$ \\
\hline & & & & & & $3.27 \times 10^{7 \mathrm{~B}}$ & \\
\hline $7 \mathrm{p}^{2} \mathrm{P}_{3 / 2}^{\mathrm{o}}$ & $10 s^{2} S_{1 / 2}$ & $2605.59^{A}$ & $2604.82^{\mathrm{a}}$ & $0.060^{\mathrm{A}, \mathrm{B}}$ & $0.064^{\mathrm{a}}$ & $5.91 \times 10^{7 \mathrm{~A}}$ & $6.25 \times 10^{7 \mathrm{a}}$ \\
\hline & & $2605.60^{\mathrm{B}}$ & $2604.827^{c}$ & & & $5.94 \times 10^{7 \mathrm{~B}}$ & \\
\hline $7 \mathrm{p}^{2} \mathrm{P}_{1 / 2}^{\mathrm{o}}$ & $9 \mathrm{~d}^{2} \mathrm{D}_{3 / 2}$ & $2514.19^{\mathrm{A}, \mathrm{B}}$ & $2513.43^{\mathrm{a}}$ & $0.083^{\mathrm{A}}$ & $0.077^{\mathrm{a}}$ & $8.72 \times 10^{7 \mathrm{~A}}$ & $8.10 \times 10^{7 a}$ \\
\hline & & & $2513.432^{c}$ & $0.088^{\mathrm{B}}$ & & $9.27 \times 10^{7 \mathrm{~B}}$ & \\
\hline $7 \mathrm{p}^{2} \mathrm{P}_{3 / 2}^{\mathrm{o}}$ & $9 \mathrm{~d}^{2} \mathrm{D}_{5 / 2}$ & $2589.64^{\mathrm{A}, \mathrm{B}}$ & $2588.86^{\mathrm{a}}$ & $0.145^{\mathrm{A}}$ & $0.134^{\mathrm{a}}$ & $1.44 \times 10^{8 \mathrm{~A}}$ & $1.33 \times 10^{8 \mathrm{a}}$ \\
\hline & & & $2588.867^{c}$ & $0.153^{\text {B }}$ & & $1.53 \times 10^{8 \mathrm{~B}}$ & \\
\hline $7 \mathrm{p}^{2} \mathrm{P}_{3 / 2}^{\mathrm{o}}$ & $9 \mathrm{~d}^{2} \mathrm{D}_{3 / 2}$ & $2594.36^{\mathrm{A}, \mathrm{B}}$ & $2593.58^{\mathrm{a}}$ & $0.016^{\mathrm{A}}$ & $0.015^{\mathrm{a}}$ & $1.59 \times 10^{7 \mathrm{~A}}$ & $1.47 \times 10^{7 \mathrm{a}}$ \\
\hline & & & & $0.017^{\mathrm{B}}$ & & $1.69 \times 10^{7 \mathrm{~B}}$ & \\
\hline
\end{tabular}


TABle 1: Continued.

\begin{tabular}{|c|c|c|c|c|c|c|c|}
\hline \multicolumn{2}{|c|}{ Transition } & \multicolumn{2}{|c|}{$\lambda$} & \multicolumn{2}{|c|}{$g f$} & \multicolumn{2}{|c|}{$g A_{k i}$} \\
\hline Lower level & Upper level & This work & Other works & This work & Other works & This work & Other works \\
\hline \multirow[t]{2}{*}{$7 d^{2} D_{3 / 2}$} & $7 f^{2} F_{5 / 2}^{o}$ & $6057.51^{\mathrm{A}}$ & $6055.85^{\mathrm{a}}$ & $0.518^{\mathrm{A}}$ & $0.604^{\mathrm{a}}$ & $0.94 \times 10^{8 \mathrm{~A}}$ & $1.10 \times 10^{8 \mathrm{a}}$ \\
\hline & & $6057.56^{\mathrm{B}}$ & $6055.838^{c}$ & $0.578^{\mathrm{B}}$ & & $1.05 \times 10^{8 \mathrm{~B}}$ & \\
\hline \multirow[t]{2}{*}{$7 d^{2} D_{5 / 2}$} & $7 \mathrm{f}^{2} \mathrm{~F}_{7 / 2}^{\mathrm{o}}$ & $6121.04^{\mathrm{A}}$ & $6119.27^{\mathrm{a}}$ & $0.735^{\mathrm{A}}$ & $0.854^{\mathrm{a}}$ & $1.31 \times 10^{8 \mathrm{~A}}$ & $1.52 \times 10^{8 \mathrm{a}}$ \\
\hline & & $6121.00^{\mathrm{B}}$ & $6119.254^{c}$ & $0.818^{\mathrm{B}}$ & & $1.46 \times 10^{8 \mathrm{~B}}$ & \\
\hline \multirow{2}{*}{$7 d^{2} D_{5 / 2}$} & $7 f^{2} F_{5 / 2}^{o}$ & $6133.39^{\mathrm{A}}$ & $6131.67^{\mathrm{a}}$ & $0.037^{\mathrm{A}}$ & $0.043^{\mathrm{a}}$ & $6.51 \times 10^{6 \mathrm{~A}}$ & $7.55 \times 10^{6 a}$ \\
\hline & & $6133.35^{\mathrm{B}}$ & & $0.041^{\mathrm{B}}$ & & $7.24 \times 10^{6 \mathrm{~B}}$ & \\
\hline \multirow[t]{2}{*}{$7 \mathrm{~d}^{2} \mathrm{D}_{3 / 2}$} & $8 \mathrm{f}^{2} \mathrm{~F}_{5 / 2}^{\mathrm{o}}$ & $4194.71^{\mathrm{A}}$ & $4193.51^{\mathrm{a}}$ & $0.260^{\mathrm{A}}$ & $0.292^{\mathrm{a}}$ & $0.99 \times 10^{8 \mathrm{~A}}$ & $1.11 \times 10^{8 \mathrm{a}}$ \\
\hline & & $4194.72^{\mathrm{B}}$ & & $0.283^{\mathrm{B}}$ & & $1.07 \times 10^{8 \mathrm{~B}}$ & \\
\hline \multirow[t]{2}{*}{$7 d^{2} D_{5 / 2}$} & $8 \mathrm{f}^{2} \mathrm{~F}_{7 / 2}^{\mathrm{o}}$ & $4226.33^{\mathrm{A}}$ & $4225.12^{\mathrm{a}}$ & $0.370^{\mathrm{A}}$ & $0.414^{\mathrm{a}}$ & $1.38 \times 10^{8 \mathrm{~A}}$ & $1.54 \times 10^{8 \mathrm{a}}$ \\
\hline & & $4226.29^{\mathrm{B}}$ & & $0.402^{\mathrm{B}}$ & & $1.50 \times 10^{8 \mathrm{~B}}$ & \\
\hline \multirow[t]{2}{*}{$7 d^{2} D_{5 / 2}$} & $8 f^{2} F_{5 / 2}^{o}$ & $4230.95^{\mathrm{A}}$ & $4229.73^{a}$ & $0.018^{\mathrm{A}}$ & $0.021^{\mathrm{a}}$ & $6.89 \times 10^{6 \mathrm{~A}}$ & $7.70 \times 10^{6 a}$ \\
\hline & & $4230.92^{\mathrm{B}}$ & & $0.020^{\mathrm{B}}$ & & $7.47 \times 10^{6 \mathrm{~B}}$ & \\
\hline \multirow[t]{2}{*}{$7 \mathrm{~d}^{2} \mathrm{D}_{3 / 2}$} & $9 \mathrm{p}^{2} \mathrm{P}_{3 / 2}^{\mathrm{o}}$ & $5746.85^{\mathrm{A}}$ & $5745.26^{\mathrm{a}}$ & $0.008^{\mathrm{A}}$ & $0.007^{\mathrm{a}}$ & $1.67 \times 10^{6 \mathrm{~A}}$ & $1.49 \times 10^{6 a}$ \\
\hline & & $5746.90^{\mathrm{B}}$ & & $0.007^{\mathrm{B}}$ & & $1.48 \times 10^{6 \mathrm{~B}}$ & \\
\hline \multirow[t]{2}{*}{$7 d^{2} D_{5 / 2}$} & $9 \mathrm{p}^{2} \mathrm{P}_{3 / 2}^{\mathrm{o}}$ & $5815.10^{\mathrm{A}}$ & $5813.45^{\mathrm{a}}$ & $0.073^{\mathrm{A}}$ & $0.066^{\mathrm{a}}$ & $1.45 \times 10^{7 \mathrm{~A}}$ & $1.30 \times 10^{7 \mathrm{a}}$ \\
\hline & & $5815.07^{\mathrm{B}}$ & $5813.447^{c}$ & $0.065^{\mathrm{B}}$ & & $1.29 \times 10^{7 \mathrm{~B}}$ & \\
\hline \multirow{2}{*}{$7 d^{2} D_{3 / 2}$} & $9 p^{2} \mathrm{P}_{1 / 2}^{o}$ & $5877.26^{\mathrm{A}}$ & $5875.63^{\mathrm{a}}$ & $0.040^{\mathrm{A}}$ & $0.036^{\mathrm{a}}$ & $7.83 \times 10^{6 \mathrm{~A}}$ & $6.98 \times 10^{6 a}$ \\
\hline & & $5877.31^{\mathrm{B}}$ & $5875.632^{c}$ & $0.036^{\mathrm{B}}$ & & $6.92 \times 10^{6 \mathrm{~B}}$ & \\
\hline \multirow[t]{2}{*}{$7 \mathrm{f}^{2} \mathrm{~F}_{5 / 2}^{\mathrm{o}}$} & $8 \mathrm{~g}^{2} \mathrm{G}_{7 / 2}$ & $8293.36^{\mathrm{A}}$ & $8291.04^{\mathrm{a}}$ & $1.513^{\mathrm{A}, \mathrm{B}}$ & $1.485^{\mathrm{a}}$ & $1.47 \times 10^{8 \mathrm{~A}, \mathrm{~B}}$ & $1.44 \times 10^{8 \mathrm{a}}$ \\
\hline & & $8293.35^{\mathrm{B}}$ & & & & & \\
\hline \multirow[t]{2}{*}{$7 \mathrm{f}^{2} \mathrm{~F}_{7 / 2}^{\mathrm{o}}$} & $8 \mathrm{~g}^{2} \mathrm{G}_{9 / 2}$ & $8315.16^{\mathrm{A}}$ & $8312.96^{\mathrm{a}}$ & $1.956^{\mathrm{A}, \mathrm{B}}$ & $1.919^{\mathrm{a}}$ & $1.89 \times 10^{8 \mathrm{~A}, \mathrm{~B}}$ & $1.85 \times 10^{8 \mathrm{a}}$ \\
\hline & & $8315.11^{\mathrm{B}}$ & & & & & \\
\hline \multirow{2}{*}{$7 f^{2} F_{7 / 2}^{o}$} & $8 \mathrm{~g}^{2} \mathrm{G}_{7 / 2}$ & $8316.03^{\mathrm{A}}$ & $8313.81^{\mathrm{a}}$ & $0.056^{\mathrm{A}, \mathrm{B}}$ & $0.055^{\mathrm{a}}$ & $5.39 \times 10^{6 \mathrm{~A}, \mathrm{~B}}$ & $5.29 \times 10^{6 a}$ \\
\hline & & $8316.04^{\mathrm{B}}$ & & & & & \\
\hline \multirow[t]{2}{*}{$5 f^{2} F_{5 / 2}^{o}$} & $7 d^{2} D_{5 / 2}$ & $5469.30^{\mathrm{A}}$ & $5467.81^{\mathrm{a}}$ & $0.037^{\mathrm{A}}$ & $0.035^{\mathrm{a}}$ & $8.26 \times 10^{6 \mathrm{~A}}$ & $7.89 \times 10^{6 a}$ \\
\hline & & $5469.35^{\mathrm{B}}$ & $5467.812^{c}$ & $0.035^{\mathrm{B}}$ & & $7.77 \times 10^{6 \mathrm{~B}}$ & \\
\hline \multirow[t]{2}{*}{$5 f^{2} F_{5 / 2}^{o}$} & $7 d^{2} D_{3 / 2}$ & $5531.09^{\mathrm{A}}$ & $5529.54^{\mathrm{a}}$ & $0.514^{\mathrm{A}}$ & $0.490^{\mathrm{a}}$ & $1.12 \times 10^{8 \mathrm{~A}}$ & $1.07 \times 10^{8 \mathrm{a}}$ \\
\hline & & $5531.07^{\mathrm{B}}$ & $5529.542^{c}$ & $0.482^{\mathrm{B}}$ & & $1.05 \times 10^{8 \mathrm{~B}}$ & \\
\hline \multirow[t]{2}{*}{$5 f^{2} \mathrm{~F}_{7 / 2}^{\mathrm{o}}$} & $7 d^{2} D_{5 / 2}$ & $5493.40^{\mathrm{A}}$ & $5491.90^{\mathrm{a}}$ & $0.738^{\mathrm{A}}$ & $0.704^{\mathrm{a}}$ & $1.63 \times 10^{8 \mathrm{~A}}$ & $1.56 \times 10^{8 \mathrm{a}}$ \\
\hline & & $5493.43^{\mathrm{B}}$ & $5491.902^{c}$ & $0.693^{\mathrm{B}}$ & & $1.53 \times 10^{8 \mathrm{~B}}$ & \\
\hline $5 f^{2} \mathrm{~F}_{5 / 2}^{o}$ & $5 g^{2} G_{7 / 2}$ & $4484.21^{\mathrm{A}}$ & $4482.98^{\mathrm{a}}$ & $8.886^{\mathrm{A}}$ & $8.277^{\mathrm{a}}$ & $2.95 \times 10^{9 \mathrm{~A}, \mathrm{~B}}$ & $2.75 \times 10^{9 a}$ \\
\hline & & $4484.25^{\mathrm{B}}$ & $4482.967^{c}$ & $8.889^{\mathrm{B}}$ & & & \\
\hline $5 f^{2} F_{7 / 2}^{o}$ & $5 g^{2} \mathrm{G}_{9 / 2}$ & $4500.32^{\mathrm{A}}$ & $4499.06^{\mathrm{a}}$ & $11.478^{\mathrm{A}}$ & $10.692^{\mathrm{a}}$ & $3.78 \times 10^{9 \mathrm{~A}, \mathrm{~B}}$ & $3.52 \times 10^{9 \mathrm{a}}$ \\
\hline & & $4500.34^{\mathrm{B}}$ & $4499.050^{c}$ & $11.482^{\mathrm{B}}$ & & & \\
\hline $5 f^{2} F_{7 / 2}^{o}$ & $5 \mathrm{~g}^{2} \mathrm{G}_{7 / 2}$ & $4500.39^{\mathrm{A}}$ & $4499.15^{\mathrm{a}}$ & $0.328^{\mathrm{A}, \mathrm{B}}$ & $0.306^{\mathrm{a}}$ & $1.08 \times 10^{8 \mathrm{~A}, \mathrm{~B}}$ & $1.01 \times 10^{8 \mathrm{a}}$ \\
\hline & & $4500.43^{\mathrm{B}}$ & & & & & \\
\hline $5 f^{2} F_{5 / 2}^{o}$ & $8 d^{2} D_{5 / 2}$ & $3086.28^{\mathrm{A}}$ & $3085.38^{a}$ & $0.004^{\mathrm{A}, \mathrm{B}}$ & $0.005^{\mathrm{a}}$ & $2.98 \times 10^{6 \mathrm{~A}}$ & $3.13 \times 10^{6 a}$ \\
\hline & & $3086.29^{\mathrm{B}}$ & $3085.379^{c}$ & & & $2.80 \times 10^{6 \mathrm{~B}}$ & \\
\hline $5 f^{2} F_{5 / 2}^{o}$ & $8 \mathrm{~d}^{2} \mathrm{D}_{3 / 2}$ & $3097.15^{\mathrm{A}}$ & $3096.26^{\mathrm{a}}$ & $0.059^{\mathrm{A}}$ & $0.062^{\mathrm{a}}$ & $4.13 \times 10^{7 \mathrm{~A}}$ & $4.33 \times 10^{7 \mathrm{a}}$ \\
\hline & & $3097.16^{\mathrm{B}}$ & $3096.255^{c}$ & $0.056^{\mathrm{B}}$ & & $3.88 \times 10^{7 \mathrm{~B}}$ & \\
\hline $5 f^{2} F_{7 / 2}^{o}$ & $8 \mathrm{~d}^{2} \mathrm{D}_{5 / 2}$ & $3093.93^{\mathrm{A}}$ & $3093.03^{\mathrm{a}}$ & $0.085^{\mathrm{A}}$ & $0.089^{\mathrm{a}}$ & $5.91 \times 10^{7 \mathrm{~A}}$ & $6.21 \times 10^{7 \mathrm{a}}$ \\
\hline & & $3093.94^{\mathrm{B}}$ & $3093.028^{c}$ & $0.080^{\mathrm{B}}$ & & $5.56 \times 10^{7 \mathrm{~B}}$ & \\
\hline $5 f^{2} \mathrm{~F}_{5 / 2}^{o}$ & $6 \mathrm{~g}^{2} \mathrm{G}_{7 / 2}$ & $2898.73^{\mathrm{A}}$ & $2897.88^{\mathrm{a}}$ & $0.866^{\mathrm{A}, \mathrm{B}}$ & $0.786^{\mathrm{a}}$ & $6.87 \times 10^{8 \mathrm{~A}, \mathrm{~B}}$ & $6.24 \times 10^{8 \mathrm{a}}$ \\
\hline & & $2898.74^{\mathrm{B}}$ & $2897.875^{c}$ & & & & \\
\hline $5 f^{2} F_{7 / 2}^{o}$ & $6 g^{2} G_{9 / 2}$ & $2905.42^{\mathrm{A}}$ & $2904.57^{a}$ & $1.120^{\mathrm{A}, \mathrm{B}}$ & $1.016^{\mathrm{a}}$ & $8.85 \times 10^{8 \mathrm{~A}, \mathrm{~B}}$ & $8.03 \times 10^{8 \mathrm{a}}$ \\
\hline & & $2905.41^{\mathrm{B}}$ & $2904.576^{c}$ & & & & \\
\hline $5 f^{2} F_{7 / 2}^{o}$ & $6 \mathrm{~g}^{2} \mathrm{G}_{7 / 2}$ & $2905.49^{\mathrm{A}, \mathrm{B}}$ & $2904.63^{\mathrm{a}}$ & $0.032^{\mathrm{A}, \mathrm{B}}$ & $0.029^{\mathrm{a}}$ & $2.53 \times 10^{7 \mathrm{~A}, \mathrm{~B}}$ & $2.29 \times 10^{7 \mathrm{a}}$ \\
\hline $5 f^{2} \mathrm{~F}_{5 / 2}^{o}$ & $9 \mathrm{~d}^{2} \mathrm{D}_{5 / 2}$ & $2461.70^{\mathrm{A}, \mathrm{B}}$ & $2460.95^{\mathrm{a}}$ & $0.001^{\mathrm{A}, \mathrm{B}}$ & $0.002^{\mathrm{a}}$ & $1.51 \times 10^{6 \mathrm{~A}}$ & $1.67 \times 10^{6 \mathrm{a}}$ \\
\hline & & & & & & $1.41 \times 10^{6 \mathrm{~B}}$ & \\
\hline $5 f^{2} F_{5 / 2}^{o}$ & $9 \mathrm{~d}^{2} \mathrm{D}_{3 / 2}$ & $2465.97^{\mathrm{A}, \mathrm{B}}$ & $2465.22^{\mathrm{a}}$ & $0.019^{\mathrm{A}}$ & $0.021^{\mathrm{a}}$ & $2.11 \times 10^{7 \mathrm{~A}}$ & $2.33 \times 10^{7 \mathrm{a}}$ \\
\hline & & & & $0.018^{\mathrm{B}}$ & & $1.96 \times 10^{7 \mathrm{~B}}$ & \\
\hline
\end{tabular}


TABle 1: Continued.

\begin{tabular}{|c|c|c|c|c|c|c|c|}
\hline \multicolumn{2}{|c|}{ Transition } & \multicolumn{2}{|c|}{$\lambda$} & \multicolumn{2}{|c|}{$g f$} & \multicolumn{2}{|c|}{$g A_{k i}$} \\
\hline Lower level & Upper level & This work & Other works & This work & Other works & This work & Other works \\
\hline \multirow{2}{*}{$5 f^{2} F_{7 / 2}^{o}$} & $9 d^{2} D_{5 / 2}$ & $2466.59^{\mathrm{A}}$ & $2465.82^{\mathrm{a}}$ & $0.027^{\mathrm{A}}$ & $0.030^{\mathrm{a}}$ & $3.01 \times 10^{7 \mathrm{~A}}$ & $3.33 \times 10^{7 \mathrm{a}}$ \\
\hline & & $2535.57^{\mathrm{B}}$ & & $0.025^{\mathrm{B}}$ & & $2.80 \times 10^{7 \mathrm{~B}}$ & \\
\hline \multirow{2}{*}{$5 f^{2} F_{5 / 2}^{o}$} & $7 g^{2} G_{7 / 2}$ & $2388.71^{\mathrm{A}}$ & $2387.99^{a}$ & $0.226^{\mathrm{A}, \mathrm{B}}$ & $0.196^{\mathrm{a}}$ & $2.64 \times 10^{8 \mathrm{~A}, \mathrm{~B}}$ & $2.29 \times 10^{8 \mathrm{a}}$ \\
\hline & & $2388.72^{\mathrm{B}}$ & $2387.988^{c}$ & & & & \\
\hline \multirow[t]{2}{*}{$5 f^{2} \mathrm{~F}_{7 / 2}^{o}$} & $7 \mathrm{~g}^{2} \mathrm{G}_{9 / 2}$ & $2393.23^{\mathrm{A}}$ & $2392.49^{a}$ & $0.292^{\mathrm{A}, \mathrm{B}}$ & $0.254^{\mathrm{a}}$ & $3.41 \times 10^{8 \mathrm{~A}, \mathrm{~B}}$ & $2.96 \times 10^{8 \mathrm{a}}$ \\
\hline & & $2393.22^{\mathrm{B}}$ & $2392.492^{c}$ & & & & \\
\hline $5 f^{2} F_{7 / 2}^{o}$ & $7 g^{2} \mathrm{G}_{7 / 2}$ & $2393.30^{\mathrm{A}, \mathrm{B}}$ & $2392.57^{a}$ & $0.008^{\mathrm{A}, \mathrm{B}}$ & $0.007^{\mathrm{a}}$ & $9.73 \times 10^{6 \mathrm{~A}, \mathrm{~B}}$ & $8.45 \times 10^{6 a}$ \\
\hline $5 f^{2} \mathrm{~F}_{5 / 2}^{o}$ & $8 \mathrm{~g}^{2} \mathrm{G}_{7 / 2}$ & $2143.81^{\mathrm{A}, \mathrm{B}}$ & $2143.13^{\mathrm{a}}$ & $0.089^{\mathrm{A}, \mathrm{B}}$ & $0.074^{\mathrm{a}}$ & $1.29 \times 10^{8 \mathrm{~A}, \mathrm{~B}}$ & $1.08 \times 10^{8 \mathrm{a}}$ \\
\hline $5 \mathrm{f}^{2} \mathrm{~F}_{7 / 2}^{\mathrm{o}}$ & $8 \mathrm{~g}^{2} \mathrm{G}_{9 / 2}$ & $2147.44^{\mathrm{A}, \mathrm{B}}$ & $2146.77^{\mathrm{a}}$ & $0.115^{\mathrm{A}, \mathrm{B}}$ & $0.096^{\mathrm{a}}$ & $1.67 \times 10^{8 \mathrm{~A}, \mathrm{~B}}$ & $1.39 \times 10^{8 \mathrm{a}}$ \\
\hline $5 \mathrm{f}^{2} \mathrm{~F}_{7 / 2}^{\mathrm{o}}$ & $8 g^{2} \mathrm{G}_{7 / 2}$ & $2147.50^{\mathrm{A}, \mathrm{B}}$ & $2146.83^{\mathrm{a}}$ & $0.003^{\mathrm{A}, \mathrm{B}}$ & $0.003^{\mathrm{a}}$ & $4.77 \times 10^{6 \mathrm{~A}, \mathrm{~B}}$ & $3.98 \times 10^{6 a}$ \\
\hline \multirow[t]{2}{*}{$5 g^{2} \mathrm{G}_{9 / 2}$} & $7 \mathrm{f}^{2} \mathrm{~F}_{7 / 2}^{\mathrm{o}}$ & $8116.78^{\mathrm{A}}$ & $8114.48^{\mathrm{a}}$ & $0.018^{\mathrm{A}, \mathrm{B}}$ & $0.021^{\mathrm{a}}$ & $1.79 \times 10^{6 \mathrm{~A}, \mathrm{~B}}$ & $2.11 \times 10^{6 a}$ \\
\hline & & $8116.75^{\mathrm{B}}$ & $8114.415^{c}$ & & & & \\
\hline \multirow[t]{2}{*}{$5 \mathrm{~g}^{2} \mathrm{G}_{7 / 2}$} & $7 \mathrm{f}^{2} \mathrm{~F}_{5 / 2}^{\mathrm{o}}$ & $8138.27^{\mathrm{A}}$ & $8136.00^{\mathrm{a}}$ & $0.014^{\mathrm{A}, \mathrm{B}}$ & $0.016^{\mathrm{a}}$ & $1.37 \times 10^{6 \mathrm{~A}, \mathrm{~B}}$ & $1.62 \times 10^{6 \mathrm{a}}$ \\
\hline & & $8138.19^{\mathrm{B}}$ & $8135.964^{c}$ & & & & \\
\hline \multirow[t]{2}{*}{$5 g^{2} \mathrm{G}_{9 / 2}$} & $8 \mathrm{f}^{2} \mathrm{~F}_{7 / 2}^{\mathrm{o}}$ & $5090.54^{\mathrm{A}}$ & $5089.12^{\mathrm{a}}$ & $0.003^{\mathrm{A}, \mathrm{B}}$ & $0.003^{\mathrm{a}}$ & $6.83 \times 10^{5 \mathrm{~A}}$ & $7.82 \times 10^{5 a}$ \\
\hline & & $5090.51^{\mathrm{B}}$ & & & & $6.86 \times 10^{5 \mathrm{~B}}$ & \\
\hline \multirow[t]{2}{*}{$5 \mathrm{~g}^{2} \mathrm{G}_{7 / 2}$} & $8 f^{2} F_{5 / 2}^{o}$ & $5097.17^{\mathrm{A}}$ & $5095.70^{\mathrm{a}}$ & $0.002^{\mathrm{A}, \mathrm{B}}$ & $0.002^{\mathrm{a}}$ & $5.25 \times 10^{5 \mathrm{~A}}$ & $6.01 \times 10^{5 \mathrm{a}}$ \\
\hline & & $5097.11^{\mathrm{B}}$ & & & & $5.27 \times 10^{5 \mathrm{~B}}$ & \\
\hline \multirow{2}{*}{$5 d^{2} D_{3 / 2}$} & $4 \mathrm{f}^{2} \mathrm{~F}_{5 / 2}^{\mathrm{o}}$ & $13898.50^{\mathrm{A}}$ & $13894.47^{\mathrm{f}}$ & $0.072^{\mathrm{A}}$ & $0.031^{\mathrm{d}}$ & $2.51 \times 10^{6 \mathrm{~A}}$ & - \\
\hline & & $13898.06^{\mathrm{B}}$ & & $0.074^{\mathrm{B}}$ & & $2.54 \times 10^{6 \mathrm{~B}}$ & \\
\hline \multirow[t]{2}{*}{$5 d^{2} D_{5 / 2}$} & $4 \mathrm{f}^{2} \mathrm{~F}_{7 / 2}^{\mathrm{o}}$ & $14099.97^{\mathrm{A}}$ & $14096.18^{f}$ & $0.102^{\mathrm{A}}$ & $0.046^{\mathrm{d}}$ & $3.44 \times 10^{6 \mathrm{~A}}$ & - \\
\hline & & $14100.19^{\mathrm{B}}$ & & $0.104^{\mathrm{B}}$ & & $3.48 \times 10^{6 \mathrm{~B}}$ & \\
\hline \multirow[t]{2}{*}{$5 d^{2} D_{5 / 2}$} & $4 \mathrm{f}^{2} \mathrm{~F}_{5 / 2}^{\mathrm{o}}$ & $17882.04^{\mathrm{A}}$ & $17878.09^{f}$ & $0.004^{\mathrm{A}, \mathrm{B}}$ & $0.002^{\mathrm{d}}$ & $8.41 \times 10^{4 \mathrm{~A}}$ & - \\
\hline & & $17883.69^{\mathrm{B}}$ & & & & $8.52 \times 10^{4 \mathrm{~B}}$ & \\
\hline \multirow[t]{3}{*}{$5 d^{2} D_{5 / 2}$} & $6 \mathrm{p}^{2} \mathrm{P}_{3 / 2}^{\mathrm{o}}$ & $2298.44^{\mathrm{A}, \mathrm{B}}$ & $2297.74^{\mathrm{a}}$ & $1.172^{\mathrm{A}}$ & $1.120^{\mathrm{a}}$ & $1.48 \times 10^{9 \mathrm{~A}}$ & $1.41 \times 10^{9 \mathrm{a}}$ \\
\hline & & & $2297.737^{c}$ & $1.315^{\mathrm{B}}$ & $1.050^{\mathrm{d}}$ & $1.66 \times 10^{9 \mathrm{~B}}$ & $1.33 \times 10^{9 b}$ \\
\hline & & & $2298.44^{\mathrm{g}}$ & & & & \\
\hline \multirow[t]{2}{*}{$5 d^{2} D_{3 / 2}$} & $6 \mathrm{p}^{2} \mathrm{P}_{3 / 2}^{\mathrm{o}}$ & $2216.76^{\mathrm{A}}$ & $2216.07^{\mathrm{a}}$ & $0.135^{\mathrm{A}}$ & $0.129^{\mathrm{a}}$ & $1.83 \times 10^{8 \mathrm{~A}}$ & $1.75 \times 10^{8 \mathrm{a}}$ \\
\hline & & $2216.75^{\mathrm{B}}$ & $2216.067^{c}$ & $0.151^{\mathrm{B}}$ & $0.115^{\mathrm{d}}$ & $2.06 \times 10^{8 \mathrm{~B}}$ & $1.56 \times 10^{8 b}$ \\
\hline \multirow[t]{3}{*}{$5 d^{2} D_{3 / 2}$} & $6 \mathrm{p}^{2} \mathrm{P}_{1 / 2}^{\mathrm{o}}$ & $2380.10^{\mathrm{A}}$ & $2379.37^{a}$ & $0.629^{\mathrm{A}}$ & $0.601^{\mathrm{a}}$ & $7.41 \times 10^{8 \mathrm{~A}}$ & $7.07 \times 10^{8 \mathrm{a}}$ \\
\hline & & $2380.09^{\mathrm{B}}$ & $2379.374^{c}$ & $0.705^{\mathrm{B}}$ & $0.576^{\mathrm{d}}$ & $8.31 \times 10^{8 \mathrm{~B}}$ & $6.78 \times 10^{8 b}$ \\
\hline & & & $2380.10^{\mathrm{g}}$ & & & & \\
\hline \multirow{2}{*}{$5 \mathrm{~d}^{2} \mathrm{D}_{3 / 2}$} & $5 f^{2} F_{5 / 2}^{o}$ & $1081.61^{\mathrm{A}, \mathrm{B}}$ & $1081.61^{\mathrm{a}}$ & $1.649^{\mathrm{A}}$ & $1.377^{\mathrm{a}}$ & $9.40 \times 10^{9 \mathrm{~A}}$ & $7.85 \times 10^{9 a}$ \\
\hline & & & & $1.640^{\mathrm{B}}$ & $1.604^{\mathrm{d}}$ & $9.35 \times 10^{9 \mathrm{~B}}$ & $9.06 \times 10^{9 b}$ \\
\hline $5 d^{2} D_{5 / 2}$ & $5 f^{2} F_{7 / 2}^{o}$ & $1099.73^{\mathrm{A}, \mathrm{B}}$ & $1099.73^{a}$ & $2.317^{\mathrm{A}}$ & $1.935^{\mathrm{a}}$ & $1.28 \times 10^{10 \mathrm{~A}}$ & $1.07 \times 10^{10 \mathrm{a}}$ \\
\hline & & & & $2.304^{\mathrm{B}}$ & $2.325^{\mathrm{d}}$ & $1.27 \times 10^{10 \mathrm{~B}}$ & $1.28 \times 10^{10 b}$ \\
\hline $5 d^{2} D_{5 / 2}$ & $5 f^{2} F_{5 / 2}^{o}$ & $1100.70^{\mathrm{A}, \mathrm{B}}$ & $1100.70^{\mathrm{a}}$ & $0.115^{\mathrm{A}, \mathrm{B}}$ & $0.097^{\mathrm{a}}$ & $6.37 \times 10^{8 \mathrm{~A}}$ & $5.32 \times 10^{8 a}$ \\
\hline & & & & & $0.119^{\mathrm{d}}$ & $6.34 \times 10^{8 \mathrm{~B}}$ & $6.49 \times 10^{8 b}$ \\
\hline $5 d^{2} D_{3 / 2}$ & $7 \mathrm{p}^{2} \mathrm{P}_{3 / 2}^{\mathrm{o}}$ & $1058.63^{\mathrm{A}, \mathrm{B}}$ & $1058.63^{a}$ & $0.013^{\mathrm{A}, \mathrm{B}}$ & $0.010^{\mathrm{a}}$ & $7.84 \times 10^{7 \mathrm{~A}}$ & $5.72 \times 10^{7 \mathrm{a}}$ \\
\hline & & & & & & $8.02 \times 10^{7 \mathrm{~B}}$ & \\
\hline $5 d^{2} D_{3 / 2}$ & $7 \mathrm{p}^{2} \mathrm{P}_{1 / 2}^{\mathrm{o}}$ & $1072.59^{\mathrm{A}, \mathrm{B}}$ & $1072.59^{\mathrm{a}}$ & $0.067^{\mathrm{A}, \mathrm{B}}$ & $0.048^{\mathrm{a}}$ & $3.77 \times 10^{8 \mathrm{~A}}$ & $2.75 \times 10^{8 \mathrm{a}}$ \\
\hline & & & & & & $3.86 \times 10^{8 \mathrm{~B}}$ & \\
\hline $5 d^{2} D_{5 / 2}$ & $7 \mathrm{p}^{2} \mathrm{P}_{3 / 2}^{\mathrm{o}}$ & $1076.91^{\mathrm{A}, \mathrm{B}}$ & $1076.91^{\mathrm{a}}$ & $0.116^{\mathrm{A}}$ & $0.085^{\mathrm{a}}$ & $6.70 \times 10^{8 \mathrm{~A}}$ & $4.89 \times 10^{8 \mathrm{a}}$ \\
\hline & & & & $0.119^{\mathrm{B}}$ & & $6.86 \times 10^{8 \mathrm{~B}}$ & \\
\hline $5 d^{2} D_{3 / 2}$ & $6 \mathrm{f}^{2} \mathrm{~F}_{5 / 2}^{\mathrm{o}}$ & $870.40^{\mathrm{A}, \mathrm{B}}$ & $870.40^{\mathrm{a}}$ & $0.614^{\mathrm{A}, \mathrm{B}}$ & $0.446^{\mathrm{a}}$ & $5.41 \times 10^{9 \mathrm{~A}}$ & $3.93 \times 10^{9 \mathrm{a}}$ \\
\hline & & & & & $0.480^{\mathrm{d}}$ & $5.40 \times 10^{9 \mathrm{~B}}$ & $4.21 \times 10^{9 b}$ \\
\hline $5 d^{2} D_{5 / 2}$ & $6 \mathrm{f}^{2} \mathrm{~F}_{7 / 2}^{\mathrm{o}}$ & $882.34^{\mathrm{A}, \mathrm{B}}$ & $882.34^{\mathrm{a}}$ & $0.865^{\mathrm{A}, \mathrm{B}}$ & $0.629^{a}$ & $7.42 \times 10^{9 \mathrm{~A}}$ & $5.39 \times 10^{9 \mathrm{a}}$ \\
\hline & & & & & $0.696^{\mathrm{d}}$ & $7.41 \times 10^{9 \mathrm{~B}}$ & $5.95 \times 10^{9 b}$ \\
\hline $5 d^{2} D_{5 / 2}$ & $6 \mathrm{f}^{2} \mathrm{~F}_{5 / 2}^{\mathrm{o}}$ & $882.72^{\mathrm{A}, \mathrm{B}}$ & $882.72^{\mathrm{a}}$ & $0.043^{\mathrm{A}, \mathrm{B}}$ & $0.031^{\mathrm{a}}$ & $3.71 \times 10^{8 \mathrm{~A}}$ & $2.69 \times 10^{8 \mathrm{a}}$ \\
\hline & & & & & $0.035^{\mathrm{d}}$ & $3.70 \times 10^{8 \mathrm{~B}}$ & $2.99 \times 10^{8 b}$ \\
\hline $5 d^{2} D_{3 / 2}$ & $8 \mathrm{p}^{2} \mathrm{P}_{3 / 2}^{\mathrm{o}}$ & $860.39^{A, B}$ & $860.39^{a}$ & $0.005^{\mathrm{A}, \mathrm{B}}$ & $0.003^{\mathrm{a}}$ & $4.24 \times 10^{7 \mathrm{~A}}$ & $2.76 \times 10^{7 \mathrm{a}}$ \\
\hline & & & & & & $4.36 \times 10^{7 \mathrm{~B}}$ & \\
\hline
\end{tabular}


Table 1: Continued.

\begin{tabular}{|c|c|c|c|c|c|c|c|}
\hline \multicolumn{2}{|c|}{ Transition } & \multicolumn{2}{|c|}{$\lambda$} & \multicolumn{2}{|c|}{$g f$} & \multicolumn{2}{|c|}{$g A_{k i}$} \\
\hline Lower level & Upper level & This work & Other works & This work & Other works & This work & Other works \\
\hline \multirow[t]{2}{*}{$5 \mathrm{~d}^{2} \mathrm{D}_{3 / 2}$} & $8 \mathrm{p}^{2} \mathrm{P}_{1 / 2}^{\mathrm{o}}$ & $865.04^{\mathrm{A}, \mathrm{B}}$ & $865.04^{\mathrm{a}}$ & $0.024^{\mathrm{A}, \mathrm{B}}$ & $0.015^{\mathrm{a}}$ & $2.08 \times 10^{8 \mathrm{~A}}$ & $1.36 \times 10^{8 \mathrm{a}}$ \\
\hline & & & & & & $2.15 \times 10^{8 \mathrm{~B}}$ & \\
\hline \multirow{2}{*}{$5 d^{2} D_{5 / 2}$} & $8 \mathrm{p}^{2} \mathrm{P}_{3 / 2}^{\mathrm{o}}$ & $872.43^{\mathrm{A}, \mathrm{B}}$ & $872.43^{\mathrm{a}}$ & $0.043^{\mathrm{A}, \mathrm{B}}$ & $0.027^{\mathrm{a}}$ & $3.65 \times 10^{8 \mathrm{~A}}$ & $2.38 \times 10^{8 \mathrm{a}}$ \\
\hline & & & & & & $3.77 \times 10^{8 \mathrm{~B}}$ & \\
\hline \multirow[t]{2}{*}{$5 d^{2} D_{3 / 2}$} & $7 f^{2} F_{5 / 2}^{o}$ & $787.14^{\mathrm{A}, \mathrm{B}}$ & $787.14^{\mathrm{a}}$ & $0.303^{\mathrm{A}}$ & $0.203^{\mathrm{a}}$ & $3.24 \times 10^{9 \mathrm{~A}}$ & $2.18 \times 10^{9 a}$ \\
\hline & & & & $0.305^{\mathrm{B}}$ & & $3.28 \times 10^{9 \mathrm{~B}}$ & \\
\hline $5 d^{2} D_{5 / 2}$ & $7 \mathrm{f}^{2} \mathrm{~F}_{7 / 2}^{\mathrm{o}}$ & $796.99^{\mathrm{A}, \mathrm{B}}$ & $796.99^{a}$ & $0.430^{\mathrm{A}, \mathrm{B}}$ & $0.286^{\mathrm{a}}$ & $4.51 \times 10^{9 \mathrm{~A}, \mathrm{~B}}$ & $3.00 \times 10^{9 \mathrm{a}}$ \\
\hline $5 d^{2} D_{5 / 2}$ & $7 \mathrm{f}^{2} \mathrm{~F}_{5 / 2}^{o}$ & $797.20^{\mathrm{A}, \mathrm{B}}$ & $797.20^{\mathrm{a}}$ & $0.021^{\mathrm{A}, \mathrm{B}}$ & $0.014^{\mathrm{a}}$ & $2.26 \times 10^{8 \mathrm{~A}, \mathrm{~B}}$ & $1.50 \times 10^{8 \mathrm{a}}$ \\
\hline \multirow{2}{*}{$5 d^{2} D_{3 / 2}$} & $9 \mathrm{p}^{2} \mathrm{P}_{3 / 2}^{\mathrm{o}}$ & $781.65^{\mathrm{A}, \mathrm{B}}$ & $781.65^{\mathrm{a}}$ & $0.002^{\mathrm{A}, \mathrm{B}}$ & $0.001^{\mathrm{a}}$ & $2.52 \times 10^{7 \mathrm{~A}}$ & $1.54 \times 10^{7 \mathrm{a}}$ \\
\hline & & & & & & $2.62 \times 10^{7 \mathrm{~B}}$ & \\
\hline \multirow{2}{*}{$5 d^{2} D_{3 / 2}$} & $9 \mathrm{p}^{2} \mathrm{P}_{1 / 2}^{\mathrm{o}}$ & $784.01^{\mathrm{A}, \mathrm{B}}$ & $784.01^{\mathrm{a}}$ & $0.012^{\mathrm{A}, \mathrm{B}}$ & $0.007^{\mathrm{a}}$ & $12.50 \times 10^{7 \mathrm{~A}}$ & $7.61 \times 10^{7 \mathrm{a}}$ \\
\hline & & & & & & $13.01 \times 10^{7 \mathrm{~B}}$ & \\
\hline \multirow[t]{2}{*}{$5 d^{2} D_{5 / 2}$} & $9 \mathrm{p}^{2} \mathrm{P}_{3 / 2}^{\mathrm{o}}$ & $791.57^{\mathrm{A}, \mathrm{B}}$ & $791.57^{\mathrm{a}}$ & $0.021^{\mathrm{A}, \mathrm{B}}$ & $0.013^{\mathrm{a}}$ & $2.19 \times 10^{8 \mathrm{~A}}$ & $1.33 \times 10^{8 \mathrm{a}}$ \\
\hline & & & & & & $2.27 \times 10^{8 \mathrm{~B}}$ & \\
\hline $5 d^{2} D_{3 / 2}$ & $8 \mathrm{f}^{2} \mathrm{~F}_{5 / 2}^{\mathrm{o}}$ & $744.19^{\mathrm{A}, \mathrm{B}}$ & $744.19^{a}$ & $0.176^{\mathrm{A}, \mathrm{B}}$ & $0.110^{\mathrm{a}}$ & $2.12 \times 10^{9 \mathrm{~A}, \mathrm{~B}}$ & $1.33 \times 10^{9 \mathrm{a}}$ \\
\hline \multirow{2}{*}{$5 d^{2} D_{5 / 2}$} & $8 \mathrm{f}^{2} \mathrm{~F}_{7 / 2}^{\mathrm{o}}$ & $753.03^{\mathrm{A}, \mathrm{B}}$ & $753.03^{\mathrm{a}}$ & $0.249^{\mathrm{A}}$ & $0.155^{\mathrm{a}}$ & $2.92 \times 10^{9 \mathrm{~A}, \mathrm{~B}}$ & $1.83 \times 10^{9 \mathrm{a}}$ \\
\hline & & & & $0.248^{\mathrm{B}}$ & & & \\
\hline $5 d^{2} D_{5 / 2}$ & $8 \mathrm{f}^{2} \mathrm{~F}_{5 / 2}^{\mathrm{o}}$ & $753.18^{\mathrm{A}, \mathrm{B}}$ & $753.18^{\mathrm{a}}$ & $0.012^{\mathrm{A}, \mathrm{B}}$ & $0.008^{\mathrm{a}}$ & $1.46 \times 10^{8 \mathrm{~A}, \mathrm{~B}}$ & $9.14 \times 10^{7 a}$ \\
\hline \multirow[t]{2}{*}{$4 \mathrm{f}^{2} \mathrm{~F}_{5 / 2}^{\mathrm{o}}$} & $6 d^{2} D_{5 / 2}$ & $1322.42^{\mathrm{A}, \mathrm{B}}$ & $1322.42^{\mathrm{a}}$ & $0.000^{\mathrm{A}, \mathrm{B}}$ & $0.002^{\mathrm{a}}$ & $0.12 \times 10^{6 \mathrm{~A}}$ & $7.00 \times 10^{6 \mathrm{a}}$ \\
\hline & & & & & & $0.17 \times 10^{6 \mathrm{~B}}$ & \\
\hline \multirow[t]{2}{*}{$4 \mathrm{f}^{2} \mathrm{~F}_{5 / 2}^{\mathrm{o}}$} & $6 \mathrm{~d}^{2} \mathrm{D}_{3 / 2}$ & $1330.04^{\mathrm{A}, \mathrm{B}}$ & $1330.04^{\mathrm{a}}$ & $0.001^{\mathrm{A}, \mathrm{B}}$ & $0.026^{\mathrm{a}}$ & $0.22 \times 10^{7 \mathrm{~A}}$ & $9.64 \times 10^{7 \mathrm{a}}$ \\
\hline & & & & & & $0.23 \times 10^{7 \mathrm{~B}}$ & \\
\hline \multirow[t]{2}{*}{$4 \mathrm{f}^{2} \mathrm{~F}_{7 / 2}^{\mathrm{o}}$} & $6 d^{2} D_{5 / 2}$ & $1349.18^{\mathrm{A}, \mathrm{B}}$ & $1349.18^{\mathrm{a}}$ & $0.001^{\mathrm{A}, \mathrm{B}}$ & $0.036^{\mathrm{a}}$ & $3.07 \times 10^{6 \mathrm{~A}}$ & $1.32 \times 10^{8 \mathrm{a}}$ \\
\hline & & & & & & $3.16 \times 10^{6 \mathrm{~B}}$ & \\
\hline \multirow[t]{2}{*}{$4 f^{2} F_{5 / 2}^{o}$} & $5 g^{2} G_{7 / 2}$ & $929.72^{\mathrm{A}, \mathrm{B}}$ & $929.72^{\mathrm{a}}$ & $0.058^{\mathrm{A}}$ & $0.040^{\mathrm{a}}$ & $4.49 \times 10^{8 \mathrm{~A}}$ & $3.06 \times 10^{8 \mathrm{a}}$ \\
\hline & & & & $0.060^{\mathrm{B}}$ & & $4.63 \times 10^{8 B}$ & \\
\hline \multirow[t]{2}{*}{$4 \mathrm{f}^{2} \mathrm{~F}_{7 / 2}^{\mathrm{o}}$} & $5 g^{2} \mathrm{G}_{9 / 2}$ & $942.86^{\mathrm{A}, \mathrm{B}}$ & $942.86^{\mathrm{a}}$ & $0.074^{\mathrm{A}}$ & $0.051^{\mathrm{a}}$ & $5.58 \times 10^{8 \mathrm{~A}}$ & $3.81 \times 10^{8 \mathrm{a}}$ \\
\hline & & & & $0.077^{\mathrm{B}}$ & & $5.76 \times 10^{8 \mathrm{~B}}$ & \\
\hline \multirow[t]{2}{*}{$4 f^{2} F_{7 / 2}^{o}$} & $5 g^{2} G_{7 / 2}$ & $942.87^{\mathrm{A}, \mathrm{B}}$ & $942.87^{\mathrm{a}}$ & $0.002^{\mathrm{A}, \mathrm{B}}$ & $0.001^{\mathrm{a}}$ & $1.60 \times 10^{7 \mathrm{~A}}$ & $1.09 \times 10^{7 \mathrm{a}}$ \\
\hline & & & & & & $1.65 \times 10^{7 \mathrm{~B}}$ & \\
\hline \multirow[t]{2}{*}{$4 \mathrm{f}^{2} \mathrm{~F}_{5 / 2}^{\mathrm{o}}$} & $6 \mathrm{~g}^{2} \mathrm{G}_{7 / 2}$ & $835.02^{\mathrm{A}, \mathrm{B}}$ & $835.02^{\mathrm{a}}$ & $0.046^{\mathrm{A}}$ & $0.030^{\mathrm{a}}$ & $4.44 \times 10^{8 \mathrm{~A}}$ & $2.87 \times 10^{8 \mathrm{a}}$ \\
\hline & & & & $0.048^{\mathrm{B}}$ & & $4.58 \times 10^{8 \mathrm{~B}}$ & \\
\hline $4 \mathrm{f}^{2} \mathrm{~F}_{7 / 2}^{\mathrm{o}}$ & $6 \mathrm{~g}^{2} \mathrm{G}_{9 / 2}$ & $845.61^{\mathrm{A}, \mathrm{B}}$ & $845.61^{\mathrm{a}}$ & $0.059^{\mathrm{A}}$ & $0.038^{\mathrm{a}}$ & $5.54 \times 10^{8 \mathrm{~A}}$ & $3.58 \times 10^{8 \mathrm{a}}$ \\
\hline & & & & $0.061^{\mathrm{B}}$ & & $5.72 \times 10^{8 \mathrm{~B}}$ & \\
\hline $4 \mathrm{f}^{2} \mathrm{~F}_{7 / 2}^{\mathrm{o}}$ & $6 \mathrm{~g}^{2} \mathrm{G}_{7 / 2}$ & $845.62^{\mathrm{A}, \mathrm{B}}$ & $845.62^{\mathrm{a}}$ & $0.002^{\mathrm{A}, \mathrm{B}}$ & $0.001^{\mathrm{a}}$ & $1.59 \times 10^{7 \mathrm{~A}}$ & $1.02 \times 10^{7 \mathrm{a}}$ \\
\hline & & & & & & $1.63 \times 10^{7 \mathrm{~B}}$ & \\
\hline $4 \mathrm{f}^{2} \mathrm{~F}_{5 / 2}^{\mathrm{o}}$ & $7 \mathrm{~g}^{2} \mathrm{G}_{7 / 2}$ & $786.64^{\mathrm{A}, \mathrm{B}}$ & $786.64^{\mathrm{a}}$ & $0.033^{\mathrm{A}}$ & $0.021^{\mathrm{a}}$ & $3.56 \times 10^{8 \mathrm{~A}}$ & $2.23 \times 10^{8 \mathrm{a}}$ \\
\hline & & & & $0.034^{\mathrm{B}}$ & & $3.68 \times 10^{8 \mathrm{~B}}$ & \\
\hline $4 \mathrm{f}^{2} \mathrm{~F}_{7 / 2}^{\mathrm{o}}$ & $7 g^{2} \mathrm{G}_{9 / 2}$ & $796.03^{\mathrm{A}, \mathrm{B}}$ & $796.03^{\mathrm{a}}$ & $0.042^{\mathrm{A}}$ & $0.026^{\mathrm{a}}$ & $4.44 \times 10^{8 \mathrm{~A}}$ & $2.78 \times 10^{8 \mathrm{a}}$ \\
\hline & & & & $0.044^{\mathrm{B}}$ & & $4.60 \times 10^{8 \mathrm{~B}}$ & \\
\hline $4 \mathrm{f}^{2} \mathrm{~F}_{5 / 2}^{\mathrm{o}}$ & $8 \mathrm{~g}^{2} \mathrm{G}_{7 / 2}$ & $758.12^{\mathrm{A}, \mathrm{B}}$ & $758.12^{\mathrm{a}}$ & $0.023^{\mathrm{A}}$ & $0.014^{\mathrm{a}}$ & $2.73 \times 10^{8 \mathrm{~A}}$ & $1.67 \times 10^{8 \mathrm{a}}$ \\
\hline & & & & $0.024^{\mathrm{B}}$ & & $2.82 \times 10^{8 \mathrm{~B}}$ & \\
\hline $4 \mathrm{f}^{2} \mathrm{~F}_{7 / 2}^{\mathrm{o}}$ & $8 g^{2} \mathrm{G}_{9 / 2}$ & $766.83^{\mathrm{A}, \mathrm{B}}$ & $766.83^{\mathrm{a}}$ & $0.030^{\mathrm{A}}$ & $0.018^{\mathrm{a}}$ & $3.41 \times 10^{8 \mathrm{~A}}$ & $2.09 \times 10^{8 \mathrm{a}}$ \\
\hline & & & & $0.031^{\mathrm{B}}$ & & $3.54 \times 10^{8 \mathrm{~B}}$ & \\
\hline $8 s^{2} S_{1 / 2}$ & $9 \mathrm{p}^{2} \mathrm{P}_{3 / 2}^{\mathrm{o}}$ & $5641.62^{\mathrm{A}, \mathrm{B}}$ & $5640.03^{a}$ & $0.005^{\mathrm{A}}$ & $0.003^{\mathrm{a}}$ & $10.20 \times 10^{5 \mathrm{~A}}$ & $5.58 \times 10^{5 \mathrm{a}}$ \\
\hline & & & & $0.004^{\mathrm{B}}$ & & $9.34 \times 10^{5 \mathrm{~B}}$ & \\
\hline $8 s^{2} S_{1 / 2}$ & $9 \mathrm{p}^{2} \mathrm{P}_{1 / 2}^{\mathrm{o}}$ & $5767.24^{\mathrm{A}}$ & $5765.63^{\mathrm{a}}$ & $0.002^{\mathrm{A}, \mathrm{B}}$ & $0.001^{\mathrm{a}}$ & $4.76 \times 10^{5 \mathrm{~A}}$ & $2.61 \times 10^{5 a}$ \\
\hline & & $5767.25^{\mathrm{B}}$ & & & & $4.37 \times 10^{5 \mathrm{~B}}$ & \\
\hline $8 \mathrm{p}^{2} \mathrm{P}_{1 / 2}^{\mathrm{o}}$ & $8 \mathrm{~d}^{2} \mathrm{D}_{3 / 2}$ & $10940.83^{\mathrm{A}}$ & $10937.898^{c}$ & $3.508^{\mathrm{A}}$ & - & $1.95 \times 10^{8 \mathrm{~A}}$ & - \\
\hline & & $10940.86^{\mathrm{B}}$ & & $3.439^{\mathrm{B}}$ & & $1.92 \times 10^{8 \mathrm{~B}}$ & \\
\hline $8 p^{2} \mathrm{P}_{1 / 2}^{o}$ & $9 \mathrm{~d}^{2} \mathrm{D}_{3 / 2}$ & $5745.68^{\mathrm{A}}$ & $5744.08^{\mathrm{a}}$ & $0.337^{\mathrm{A}}$ & $0.334^{\mathrm{a}}$ & $6.81 \times 10^{7 \mathrm{~A}}$ & $6.75 \times 10^{7 a}$ \\
\hline & & $5745.66^{\mathrm{B}}$ & $5744.088^{c}$ & $0.347^{\mathrm{B}}$ & & $7.01 \times 10^{7 \mathrm{~B}}$ & \\
\hline
\end{tabular}


TABle 1: Continued.

\begin{tabular}{|c|c|c|c|c|c|c|c|}
\hline \multicolumn{2}{|c|}{ Transition } & \multicolumn{2}{|c|}{$\lambda$} & \multicolumn{2}{|c|}{$g f$} & \multicolumn{2}{|c|}{$g A_{k i}$} \\
\hline Lower level & Upper level & This work & Other works & This work & Other works & This work & Other works \\
\hline \multirow[t]{2}{*}{$8 \mathrm{p}^{2} \mathrm{P}_{3 / 2}^{\mathrm{o}}$} & $9 d^{2} D_{5 / 2}$ & $5934.37^{\mathrm{A}, \mathrm{B}}$ & $5932.73^{\mathrm{a}}$ & $0.588^{\mathrm{A}}$ & $0.582^{\mathrm{a}}$ & $1.12 \times 10^{8 \mathrm{~A}}$ & $1.10 \times 10^{8 \mathrm{a}}$ \\
\hline & & & $5932.706^{c}$ & $0.604^{\mathrm{B}}$ & & $1.14 \times 10^{8 \mathrm{~B}}$ & \\
\hline \multirow[t]{2}{*}{$8 \mathrm{p}^{2} \mathrm{P}_{3 / 2}^{\mathrm{o}}$} & $9 d^{2} D_{3 / 2}$ & $5959.22^{\mathrm{A}}$ & $5957.57^{a}$ & $0.065^{\mathrm{A}}$ & $0.064^{\mathrm{a}}$ & $1.22 \times 10^{7 \mathrm{~A}}$ & $1.21 \times 10^{7 \mathrm{a}}$ \\
\hline & & $5959.21^{\mathrm{B}}$ & & $0.067^{\mathrm{B}}$ & & $1.26 \times 10^{7 \mathrm{~B}}$ & \\
\hline \multirow[t]{2}{*}{$8 \mathrm{p}^{2} \mathrm{P}_{1 / 2}^{\mathrm{o}}$} & $10 \mathrm{~s}^{2} \mathrm{~S}_{1 / 2}$ & $5801.06^{\mathrm{A}}$ & $5799.48^{\mathrm{a}}$ & $0.112^{\mathrm{A}}$ & $0.115^{\mathrm{a}}$ & $2.23 \times 10^{7 \mathrm{~A}}$ & $2.27 \times 10^{7 \mathrm{a}}$ \\
\hline & & $5801.07^{\mathrm{B}}$ & & $0.113^{\mathrm{B}}$ & & $2.24 \times 10^{7 \mathrm{~B}}$ & \\
\hline \multirow[t]{2}{*}{$8 \mathrm{p}^{2} \mathrm{P}_{3 / 2}^{\mathrm{o}}$} & $10 \mathrm{~s}^{2} \mathrm{~S}_{1 / 2}$ & $6018.81^{\mathrm{A}}$ & $6017.18^{\mathrm{a}}$ & $0.217^{\mathrm{A}}$ & $0.221^{\mathrm{a}}$ & $3.99 \times 10^{7 \mathrm{~A}}$ & $4.06 \times 10^{7 \mathrm{a}}$ \\
\hline & & $6018.84^{\mathrm{B}}$ & $6017.114^{c}$ & $0.218^{\mathrm{B}}$ & & $4.01 \times 10^{7 \mathrm{~B}}$ & \\
\hline
\end{tabular}

${ }^{\mathrm{a}}$ Reference [1], ${ }^{\mathrm{b}}$ reference (in [1]), ${ }^{\mathrm{c}}$ reference [2], ${ }^{\mathrm{d}}$ reference [3, RMP + EX + CP ], ${ }^{\mathrm{e}}$ reference [4, RHF + CP (a)], ${ }^{\mathrm{f}}$ reference [5], ${ }^{\mathrm{g}}$ reference [6].

probabilities, for electric dipole transitions $(E 1)$ in La III $(Z=57)$. These calculations have been performed by using code [15] developed Cowan for relativistic Hartree-Fock (HFR) [16] calculations. This code considers the correlation effects and relativistic corrections. These effects contribute importantly to the physical and chemical properties of atoms or ions, especially lanthanides. The ground-state level of doubly ionized lanthanum is $[\mathrm{Xe}] 5 \mathrm{~d}^{2} \mathrm{D}_{3 / 2}$. We have taken into account $5 \mathrm{p}^{6} \mathrm{nd}, 5 \mathrm{p}^{6} \mathrm{ng}(n=5-10), 5 \mathrm{p}^{6} \mathrm{~ns}(n=6-10)$, $5 \mathrm{p}^{5} 6 \mathrm{~s} 6 \mathrm{p}, 5 \mathrm{p}^{5} 6 \mathrm{~s} 4 \mathrm{f}, 5 \mathrm{p}^{5} 5 \mathrm{~d} 6 \mathrm{p}, 5 \mathrm{p}^{6} \mathrm{nf}(n=4-10)$, 5 $\mathrm{p}^{6} \mathrm{np}(n=6-$ 10), $5 \mathrm{p}^{5} 4 \mathrm{f}^{2}$, and $5 \mathrm{p}^{5} 6 \mathrm{p}^{2}$ configurations outside the core $[\mathrm{Cd}]$, and nd, ng $(n=5-25)$, ns $(n=6-24)$, nf $(n=4-22)$, and $\mathrm{np}(n=6-25)$ configurations outside the core [Xe] in La III. The configuration sets that we used have been denoted by $\mathrm{A}$ and $\mathrm{B}$, respectively, and are given in tables and text. We presented the energies, the Landé $g$-factors, and the lifetimes for nd, ng $(n=5-25)$, ns $(n=6-24)$, $\mathrm{nf}(n=4-22)$, and $\mathrm{np}$ $(n=6-25)$ excited levels of La III [14]. In addition, we have reported various atomic structure calculations such as energy levels, transition energies, hyperfine structure, lifetimes, and electric dipole transitions for some lanthanides (La I-III, Lu I-III, and Yb I-III) [17-27].

\section{Calculation Method}

An electromagnetic transition between two states is characterized by the angular momentum and the parity of the corresponding photon. If the emitted or absorbed photon has angular momentum $k$ and parity $\pi=(-1)^{k}$ then, the transition is an electric multipole transition $(E k)$. However, if the photon has parity $\pi=(-1)^{k+1}$ the transition is a magnetic multipole transition $(M k)$.

According to HFR method [16], the total transition probability from a state $\gamma^{\prime} J^{\prime} M^{\prime}$ to all states $M$ levels of $\gamma J$ is given by

$$
A=\frac{64 \pi^{4} e^{2} a_{0}^{2} \sigma^{3}}{3 h} \mathbf{S} \sum_{M q}\left(\begin{array}{ccc}
J & 1 & J^{\prime} \\
-M & q & M^{\prime}
\end{array}\right)^{2}=\frac{64 \pi^{4} e^{2} a_{0}^{2} \sigma^{3}}{3 h\left(2 J^{\prime}+1\right)} \mathbf{S}
$$

and absorption oscillator strength is given by

$$
f_{i j}=\frac{8 \pi^{2} m c a_{0}^{2} \sigma}{3 h(2 J+1)} \mathbf{S}=\frac{\left(E_{j}-E_{i}\right)}{3(2 J+1)} \mathbf{S},
$$

where, $\sigma=\left[\left(E_{j}-E_{i}\right) / h c\right]$ has units of kaysers $\left(\mathrm{cm}^{-1}\right)$ and $\mathbf{S}=$ $\left|\left\langle\gamma J\left\|\mathbf{P}^{(1)}\right\| \gamma^{\prime} J^{\prime}\right\rangle\right|^{2}$ is the electric dipole line strength in atomic units of $e^{2} a_{0}^{2}$. The strongest transition rate (or probability) is electric dipole $(E 1)$ radiation. For this reason, the $E 1$ transitions are understood as being "allowed", whereas highorder transitions are understood as being "forbidden".

In HFR method, for an $N$ electron atom of nuclear charge $Z_{0}$, the Hamiltonian is expanded as

$$
H=-\sum_{i} \nabla_{i}^{2}-\sum_{i} \frac{2 Z_{0}}{r_{i}}+\sum_{i>j} \frac{2}{r_{i j}}+\sum_{i} \zeta_{i}\left(r_{i}\right) \mathbf{l}_{i} \cdot \mathbf{s}_{i},
$$

in atomic units, with $r_{i}$ the distance of the $i$ th electron from the nucleus and $r_{i j}=\left|\mathbf{r}_{i}-\mathbf{r}_{j}\right| . \zeta_{i}(R)=\left(\alpha^{2} / 2\right)(1 / r)(\partial V / \partial r)$ is the spin-orbit term, with $\alpha$ being the fine structure constant and $V$ the mean potential field due to the nucleus and other electrons.

In this method, one calculates single-configuration radial functions for a spherically symmetrised atom (center-ofgravity energy of the configuration) based on Hartree-Fock method. The radial wave functions are also used to obtain the atom's total energy $\left(E_{\mathrm{av}}\right)$ including approximate relativistic and correlation energy corrections. Relativistic terms in the potential function give approximate relativistic corrections to the radial functions, as well as improved relativistic energy corrections in heavy atoms. In addition, a correlation term is included to make the potential function more negative, thereby helping to bind negative ions. These radial functions are also used to calculate Coulomb integrals $F^{k}$ and $G^{k}$ and spin-orbit integrals $\zeta_{n l}$. After radial functions have been obtained based on Hartree-Fock model, the wave function $|\gamma J M\rangle$ of the $M$ sublevel of a level labeled $\gamma J$ is expressed in terms of $L S$ basis states $|\alpha L S J M\rangle$ by the formula

$$
|\gamma J M\rangle=\sum_{\alpha L S}|\alpha L S J M\rangle\langle\alpha L S J \mid \gamma J\rangle .
$$

If determinant wave functions are used for the atom, the total binding energy is given by

$$
E=\sum_{i}\left(E_{k}^{i}+E_{n}^{i}+\sum_{j<i} E^{i j}\right),
$$

where $E_{k}^{i}$ is the kinetic energy, $E_{n}^{i}$ is the electron-nuclear Coulomb energy, and $E^{i j}$ is the Coulomb interaction energy 
TAble 2: New $\lambda(\AA), g f$, and $g A_{k i}\left(\mathrm{~s}^{-1}\right)$ for electric dipole (E1) transitions in La III.

\begin{tabular}{|c|c|c|c|c|}
\hline \multicolumn{2}{|c|}{ Transition } & \multirow{2}{*}{$\lambda$} & \multirow{2}{*}{$g f$} & \multirow{2}{*}{$g A_{k i}$} \\
\hline Lower level & Upper level & & & \\
\hline $5 \mathrm{~d}^{2} \mathrm{D}_{3 / 2}$ & $12 \mathrm{f}^{2} \mathrm{~F}_{5 / 2}^{\mathrm{o}}$ & $682.99^{\mathrm{B}}$ & $0.039^{\mathrm{B}}$ & $5.62 \times 10^{8 B}$ \\
\hline $5 d^{2} D_{5 / 2}$ & $12 \mathrm{f}^{2} \mathrm{~F}_{7 / 2}^{\mathrm{o}}$ & $690.53^{\mathrm{B}}$ & $0.056^{\mathrm{B}}$ & $7.77 \times 10^{8 \mathrm{~B}}$ \\
\hline $5 d^{2} D_{5 / 2}$ & $12 \mathrm{f}^{2} \mathrm{~F}_{5 / 2}^{\mathrm{o}}$ & $690.55^{\mathrm{B}}$ & $0.003^{\mathrm{B}}$ & $3.89 \times 10^{7 \mathrm{~B}}$ \\
\hline $5 d^{2} D_{3 / 2}$ & $11 f^{2} \mathrm{~F}_{5 / 2}^{\mathrm{o}}$ & $690.98^{\mathrm{B}}$ & $0.053^{\mathrm{B}}$ & $7.46 \times 10^{8 \mathrm{~B}}$ \\
\hline $5 d^{2} D_{5 / 2}$ & $11 \mathrm{f}^{2} \mathrm{~F}_{7 / 2}^{\mathrm{o}}$ & $698.69^{\mathrm{B}}$ & $0.075^{\mathrm{B}}$ & $1.03 \times 10^{9 \mathrm{~B}}$ \\
\hline $5 d^{2} D_{5 / 2}$ & $11 f^{2} F_{5 / 2}^{o}$ & $698.72^{\mathrm{B}}$ & $0.004^{\mathrm{B}}$ & $5.15 \times 10^{7 \mathrm{~B}}$ \\
\hline $5 d^{2} D_{3 / 2}$ & $12 \mathrm{p}^{2} \mathrm{P}_{3 / 2}^{\mathrm{o}}$ & $701.16^{\mathrm{B}}$ & $0.001^{\mathrm{B}}$ & $8.21 \times 10^{6 \mathrm{~B}}$ \\
\hline $5 d^{2} D_{3 / 2}$ & $12 \mathrm{p}^{2} \mathrm{P}_{1 / 2}^{\mathrm{o}}$ & $701.60^{\mathrm{B}}$ & $0.003^{\mathrm{B}}$ & $4.10 \times 10^{7 \mathrm{~B}}$ \\
\hline \multirow{2}{*}{$5 d^{2} D_{3 / 2}$} & $10 \mathrm{f}^{2} \mathrm{~F}_{5 / 2}^{\mathrm{o}}$ & $702.10^{\mathrm{A}, \mathrm{B}}$ & $0.074^{\mathrm{A}}$ & $1.00 \times 10^{9 \mathrm{~A}}$ \\
\hline & & & $0.075^{\mathrm{B}}$ & $1.02 \times 10^{9 \mathrm{~B}}$ \\
\hline $5 d^{2} D_{5 / 2}$ & $12 \mathrm{p}^{2} \mathrm{P}_{3 / 2}^{\mathrm{o}}$ & $709.13^{\mathrm{B}}$ & $0.005^{\mathrm{B}}$ & $7.14 \times 10^{7 \mathrm{~B}}$ \\
\hline \multirow[t]{2}{*}{$5 d^{2} D_{5 / 2}$} & $10 \mathrm{f}^{2} \mathrm{~F}_{7 / 2}^{\mathrm{o}}$ & $710.05^{\mathrm{A}, \mathrm{B}}$ & $0.104^{\mathrm{A}}$ & $1.38 \times 10^{9 \mathrm{~A}}$ \\
\hline & & & $0.106^{\mathrm{B}}$ & $1.41 \times 10^{9 \mathrm{~B}}$ \\
\hline \multirow[t]{2}{*}{$5 d^{2} D_{5 / 2}$} & $10 \mathrm{f}^{2} \mathrm{~F}_{5 / 2}^{\mathrm{o}}$ & $710.09^{\mathrm{A}, \mathrm{B}}$ & $0.005^{\mathrm{A}, \mathrm{B}}$ & $6.92 \times 10^{7 \mathrm{~A}}$ \\
\hline & & & & $7.03 \times 10^{7 \mathrm{~B}}$ \\
\hline $5 d^{2} D_{3 / 2}$ & $11 \mathrm{p}^{2} \mathrm{P}_{3 / 2}^{\mathrm{o}}$ & $716.83^{\mathrm{B}}$ & $0.001^{\mathrm{B}}$ & $1.15 \times 10^{7 \mathrm{~B}}$ \\
\hline $5 d^{2} D_{3 / 2}$ & $11 \mathrm{p}^{2} \mathrm{P}_{1 / 2}^{\mathrm{o}}$ & $717.49^{\mathrm{B}}$ & $0.004^{\mathrm{B}}$ & $5.76 \times 10^{7 \mathrm{~B}}$ \\
\hline $5 d^{2} D_{5 / 2}$ & $11 \mathrm{p}^{2} \mathrm{P}_{3 / 2}^{\mathrm{o}}$ & $725.17^{\mathrm{B}}$ & $0.008^{\mathrm{B}}$ & $1.00 \times 10^{8 \mathrm{~B}}$ \\
\hline \multirow{2}{*}{$4 \mathrm{f}^{2} \mathrm{~F}_{5 / 2}^{\mathrm{o}}$} & $10 \mathrm{~g}^{2} \mathrm{G}_{7 / 2}$ & $729.02^{\mathrm{A}}$ & $0.013^{\mathrm{A}, \mathrm{B}}$ & $1.57 \times 10^{8 \mathrm{~A}}$ \\
\hline & & $727.97^{\mathrm{B}}$ & & $1.67 \times 10^{8 \mathrm{~B}}$ \\
\hline \multirow[t]{2}{*}{$4 \mathrm{f}^{2} \mathrm{~F}_{7 / 2}^{\mathrm{o}}$} & $10 \mathrm{~g}^{2} \mathrm{G}_{7 / 2}$ & $737.09^{\mathrm{A}}$ & $0.001^{\mathrm{A}, \mathrm{B}}$ & $5.70 \times 10^{6 \mathrm{~A}}$ \\
\hline & & $736.01^{\mathrm{B}}$ & & $5.97 \times 10^{6 \mathrm{~B}}$ \\
\hline \multirow[t]{2}{*}{$4 \mathrm{f}^{2} \mathrm{~F}_{7 / 2}^{\mathrm{o}}$} & $10 \mathrm{~g}^{2} \mathrm{G}_{9 / 2}$ & $737.09^{\mathrm{A}}$ & $0.016^{\mathrm{A}}$ & $1.97 \times 10^{8 \mathrm{~A}}$ \\
\hline & & $736.01^{\mathrm{B}}$ & $0.017^{\mathrm{B}}$ & $2.09 \times 10^{8 \mathrm{~B}}$ \\
\hline \multirow[t]{2}{*}{$4 \mathrm{f}^{2} \mathrm{~F}_{5 / 2}^{\mathrm{o}}$} & $9 \mathrm{~g}^{2} \mathrm{G}_{7 / 2}$ & $742.58^{\mathrm{A}}$ & $0.017^{\mathrm{A}}$ & $2.06 \times 10^{8 \mathrm{~A}}$ \\
\hline & & $740.62^{\mathrm{B}}$ & $0.018^{\mathrm{B}}$ & $2.15 \times 10^{8 \mathrm{~B}}$ \\
\hline \multirow[t]{2}{*}{$5 \mathrm{~d}^{2} \mathrm{D}_{3 / 2}$} & $10 \mathrm{p}^{2} \mathrm{P}_{3 / 2}^{\mathrm{o}}$ & $740.94^{\mathrm{A}}$ & $0.001^{\mathrm{A}, \mathrm{B}}$ & $1.60 \times 10^{7 \mathrm{~A}}$ \\
\hline & & $740.93^{\mathrm{B}}$ & & $1.70 \times 10^{7 \mathrm{~B}}$ \\
\hline \multirow[t]{2}{*}{$5 d^{2} D_{3 / 2}$} & $10 \mathrm{p}^{2} \mathrm{P}_{1 / 2}^{\mathrm{o}}$ & $742.00^{\mathrm{A}}$ & $0.007^{\mathrm{A}, \mathrm{B}}$ & $7.99 \times 10^{7 \mathrm{~A}}$ \\
\hline & & $741.97^{\mathrm{B}}$ & & $8.44 \times 10^{7 \mathrm{~B}}$ \\
\hline \multirow[t]{2}{*}{$4 \mathrm{f}^{2} \mathrm{~F}_{7 / 2}^{\mathrm{o}}$} & $9 \mathrm{~g}^{2} \mathrm{G}_{7 / 2}$ & $750.95^{\mathrm{A}}$ & $0.001^{\mathrm{A}, \mathrm{B}}$ & $7.44 \times 10^{6 \mathrm{~A}}$ \\
\hline & & $748.94^{\mathrm{B}}$ & & $7.72 \times 10^{6 \mathrm{~B}}$ \\
\hline \multirow[t]{2}{*}{$4 \mathrm{f}^{2} \mathrm{~F}_{7 / 2}^{\mathrm{o}}$} & $9 g^{2} \mathrm{G}_{9 / 2}$ & $750.95^{\mathrm{A}}$ & $0.022^{\mathrm{A}}$ & $2.58 \times 10^{8 \mathrm{~A}}$ \\
\hline & & $748.94^{\mathrm{B}}$ & $0.023^{\mathrm{B}}$ & $2.70 \times 10^{8 \mathrm{~B}}$ \\
\hline \multirow[t]{2}{*}{$5 d^{2} D_{5 / 2}$} & $10 \mathrm{p}^{2} \mathrm{P}_{3 / 2}^{\mathrm{o}}$ & $749.86^{\mathrm{A}}$ & $0.012^{\mathrm{A}, \mathrm{B}}$ & $1.39 \times 10^{8 \mathrm{~A}}$ \\
\hline & & $749.84^{\mathrm{B}}$ & & $1.47 \times 10^{8 \mathrm{~B}}$ \\
\hline $6 \mathrm{p}^{2} \mathrm{P}_{1 / 2}^{\mathrm{o}}$ & $12 \mathrm{~d}^{2} \mathrm{D}_{3 / 2}$ & $978.38^{\mathrm{B}}$ & $0.006^{\mathrm{B}}$ & $4.54 \times 10^{7 \mathrm{~B}}$ \\
\hline $6 \mathrm{p}^{2} \mathrm{P}_{1 / 2}^{\mathrm{o}}$ & $11 \mathrm{~d}^{2} \mathrm{D}_{3 / 2}$ & $1002.77^{\mathrm{B}}$ & $0.010^{\mathrm{B}}$ & $6.50 \times 10^{7 \mathrm{~B}}$ \\
\hline $6 \mathrm{p}^{2} \mathrm{P}_{3 / 2}^{\mathrm{o}}$ & $12 \mathrm{~d}^{2} \mathrm{D}_{5 / 2}$ & $1008.75^{\mathrm{B}}$ & $0.011^{\mathrm{B}}$ & $7.46 \times 10^{7 \mathrm{~B}}$ \\
\hline $6 \mathrm{p}^{2} \mathrm{P}_{3 / 2}^{\mathrm{o}}$ & $12 \mathrm{~d}^{2} \mathrm{D}_{3 / 2}$ & $1008.94^{\mathrm{B}}$ & $0.001^{\mathrm{B}}$ & $8.29 \times 10^{6 \mathrm{~B}}$ \\
\hline $6 \mathrm{p}^{2} \mathrm{P}_{3 / 2}^{\mathrm{o}}$ & $11 \mathrm{~d}^{2} \mathrm{D}_{5 / 2}$ & $1034.62^{\mathrm{B}}$ & $0.017^{\mathrm{B}}$ & $1.06 \times 10^{8 \mathrm{~B}}$ \\
\hline $6 \mathrm{p}^{2} \mathrm{P}_{3 / 2}^{\mathrm{o}}$ & $11 \mathrm{~d}^{2} \mathrm{D}_{3 / 2}$ & $1034.89^{\mathrm{B}}$ & $0.002^{\mathrm{B}}$ & $1.18 \times 10^{7 \mathrm{~B}}$ \\
\hline \multirow[t]{2}{*}{$6 \mathrm{p}^{2} \mathrm{P}_{1 / 2}^{\mathrm{o}}$} & $10 \mathrm{~d}^{2} \mathrm{D}_{3 / 2}$ & $1061.80^{\mathrm{A}}$ & $0.008^{\mathrm{A}}$ & $4.59 \times 10^{7 \mathrm{~A}}$ \\
\hline & & $1039.29^{\mathrm{B}}$ & $0.016^{\mathrm{B}}$ & $9.83 \times 10^{7 \mathrm{~B}}$ \\
\hline \multirow[t]{2}{*}{$6 p^{2} \mathrm{P}_{3 / 2}^{\mathrm{o}}$} & $10 \mathrm{~d}^{2} \mathrm{D}_{5 / 2}$ & $1097.43^{\mathrm{A}}$ & $0.014^{\mathrm{A}}$ & $7.83 \times 10^{7 \mathrm{~A}}$ \\
\hline & & $1073.42^{\mathrm{B}}$ & $0.028^{\mathrm{B}}$ & $1.61 \times 10^{8 \mathrm{~B}}$ \\
\hline \multirow[t]{2}{*}{$6 \mathrm{p}^{2} \mathrm{P}_{3 / 2}^{\mathrm{o}}$} & $10 \mathrm{~d}^{2} \mathrm{D}_{3 / 2}$ & $1097.89^{\mathrm{A}}$ & $0.002^{\mathrm{A}}$ & $8.55 \times 10^{6 \mathrm{~A}}$ \\
\hline & & $1073.84^{\mathrm{B}}$ & $0.003^{\mathrm{B}}$ & $1.78 \times 10^{7 \mathrm{~B}}$ \\
\hline $6 d^{2} D_{3 / 2}$ & $12 \mathrm{f}^{2} \mathrm{~F}_{5 / 2}^{\mathrm{o}}$ & $1561.65^{\mathrm{B}}$ & $0.036^{\mathrm{B}}$ & $9.96 \times 10^{7 \mathrm{~B}}$ \\
\hline $6 d^{2} D_{5 / 2}$ & $12 \mathrm{f}^{2} \mathrm{~F}_{7 / 2}^{\mathrm{o}}$ & $1572.18^{\mathrm{B}}$ & $0.052^{\mathrm{B}}$ & $1.39 \times 10^{8 \mathrm{~B}}$ \\
\hline $6 d^{2} D_{5 / 2}$ & $12 \mathrm{f}^{2} \mathrm{~F}_{5 / 2}^{\mathrm{o}}$ & $1572.29^{\mathrm{B}}$ & $0.002^{\mathrm{B}}$ & $6.97 \times 10^{6 \mathrm{~B}}$ \\
\hline
\end{tabular}


TABle 2: Continued.

\begin{tabular}{|c|c|c|c|c|}
\hline \multicolumn{2}{|c|}{ Transition } & \multirow{2}{*}{$\lambda$} & \multirow{2}{*}{$g f$} & \multirow{2}{*}{$g A_{k i}$} \\
\hline Lower level & Upper level & & & \\
\hline $6 \mathrm{~d}^{2} \mathrm{D}_{3 / 2}$ & $11 \mathrm{f}^{2} \mathrm{~F}_{5 / 2}^{\mathrm{o}}$ & $1604.09^{\mathrm{B}}$ & $0.050^{\mathrm{B}}$ & $1.31 \times 10^{8 B}$ \\
\hline $6 \mathrm{~d}^{2} \mathrm{D}_{5 / 2}$ & $11 \mathrm{f}^{2} \mathrm{~F}_{7 / 2}^{\mathrm{o}}$ & $1615.16^{\mathrm{B}}$ & $0.072^{\mathrm{B}}$ & $1.83 \times 10^{8 \mathrm{~B}}$ \\
\hline $6 d^{2} D_{5 / 2}$ & $11 f^{2} \mathrm{~F}_{5 / 2}^{\mathrm{o}}$ & $1615.32^{\mathrm{B}}$ & $0.004^{\mathrm{B}}$ & $9.15 \times 10^{6 B}$ \\
\hline $6 \mathrm{~d}^{2} \mathrm{D}_{3 / 2}$ & $12 \mathrm{p}^{2} \mathrm{P}_{1 / 2}^{\mathrm{o}}$ & $1662.51^{\mathrm{B}}$ & $0.002^{\mathrm{B}}$ & $5.62 \times 10^{6 \mathrm{~B}}$ \\
\hline \multirow[t]{2}{*}{$6 \mathrm{~d}^{2} \mathrm{D}_{3 / 2}$} & $10 \mathrm{f}^{2} \mathrm{~F}_{5 / 2}^{\mathrm{o}}$ & $1665.31^{\mathrm{A}}$ & $0.080^{\mathrm{A}}$ & $1.93 \times 10^{8 \mathrm{~A}}$ \\
\hline & & $1665.37^{\mathrm{B}}$ & $0.073^{\mathrm{B}}$ & $1.76 \times 10^{8 \mathrm{~B}}$ \\
\hline $6 \mathrm{~d}^{2} \mathrm{D}_{5 / 2}$ & $12 \mathrm{p}^{2} \mathrm{P}_{3 / 2}^{\mathrm{o}}$ & $1672.06^{\mathrm{B}}$ & $0.004^{\mathrm{B}}$ & $9.94 \times 10^{6 \mathrm{~B}}$ \\
\hline \multirow[t]{2}{*}{$6 d^{2} D_{5 / 2}$} & $10 \mathrm{f}^{2} \mathrm{~F}_{7 / 2}^{\mathrm{o}}$ & $1677.17^{\mathrm{A}, \mathrm{B}}$ & $0.114^{\mathrm{A}}$ & $2.70 \times 10^{8 \mathrm{~A}}$ \\
\hline & & & $0.104^{\mathrm{B}}$ & $2.46 \times 10^{8 \mathrm{~B}}$ \\
\hline \multirow{2}{*}{$6 d^{2} D_{5 / 2}$} & $10 \mathrm{f}^{2} \mathrm{~F}_{5 / 2}^{\mathrm{o}}$ & $1677.42^{\mathrm{A}, \mathrm{B}}$ & $0.006^{\mathrm{A}}$ & $1.35 \times 10^{7 \mathrm{~A}}$ \\
\hline & & & $0.005^{\mathrm{B}}$ & $1.23 \times 10^{7 \mathrm{~B}}$ \\
\hline $6 d^{2} D_{3 / 2}$ & $11 p^{2} \mathrm{P}_{1 / 2}^{\mathrm{o}}$ & $1754.58^{\mathrm{B}}$ & $0.004^{\mathrm{B}}$ & $7.85 \times 10^{6 \mathrm{~B}}$ \\
\hline $6 \mathrm{~d}^{2} \mathrm{D}_{5 / 2}$ & $11 \mathrm{p}^{2} \mathrm{P}_{3 / 2}^{\mathrm{o}}$ & $1764.04^{\mathrm{B}}$ & $0.006^{\mathrm{B}}$ & $1.39 \times 10^{7 \mathrm{~B}}$ \\
\hline \multirow{2}{*}{$6 d^{2} D_{3 / 2}$} & $10 \mathrm{p}^{2} \mathrm{P}_{3 / 2}^{\mathrm{o}}$ & $1901.87^{\mathrm{A}}$ & $0.002^{\mathrm{A}}$ & $2.94 \times 10^{6 \mathrm{~A}}$ \\
\hline & & $1901.69^{\mathrm{B}}$ & $0.001^{\mathrm{B}}$ & $2.31 \times 10^{6 \mathrm{~B}}$ \\
\hline \multirow[t]{2}{*}{$6 \mathrm{~d}^{2} \mathrm{D}_{3 / 2}$} & $10 \mathrm{p}^{2} \mathrm{P}_{1 / 2}^{\mathrm{o}}$ & $1908.76^{\mathrm{A}}$ & $0.008^{\mathrm{A}}$ & $1.45 \times 10^{7 \mathrm{~A}}$ \\
\hline & & $1908.57^{\mathrm{B}}$ & $0.006^{\mathrm{B}}$ & $1.14 \times 10^{7 \mathrm{~B}}$ \\
\hline \multirow[t]{2}{*}{$6 d^{2} D_{5 / 2}$} & $10 \mathrm{p}^{2} \mathrm{P}_{3 / 2}^{\mathrm{o}}$ & $1917.68^{\mathrm{A}}$ & $0.014^{\mathrm{A}}$ & $2.57 \times 10^{7 \mathrm{~A}}$ \\
\hline & & $1917.49^{\mathrm{B}}$ & $0.011^{\mathrm{B}}$ & $2.03 \times 10^{7 \mathrm{~B}}$ \\
\hline \multirow[t]{2}{*}{$5 f^{2} F_{5 / 2}^{o}$} & $10 \mathrm{~g}^{2} \mathrm{G}_{7 / 2}$ & $1926.41^{\mathrm{A}}$ & $0.025^{\mathrm{A}, \mathrm{B}}$ & $4.50 \times 10^{7 \mathrm{~A}}$ \\
\hline & & $1919.09^{\mathrm{B}}$ & & $4.53 \times 10^{7 \mathrm{~B}}$ \\
\hline \multirow[t]{2}{*}{$5 f^{2} F_{7 / 2}^{o}$} & $10 \mathrm{~g}^{2} \mathrm{G}_{7 / 2}$ & $1929.39^{\mathrm{A}}$ & $0.001^{\mathrm{A}, \mathrm{B}}$ & $1.66 \times 10^{6 \mathrm{~A}}$ \\
\hline & & $1922.05^{\mathrm{B}}$ & & $1.67 \times 10^{6 \mathrm{~B}}$ \\
\hline \multirow[t]{2}{*}{$5 f^{2} F_{7 / 2}^{o}$} & $10 g^{2} \mathrm{G}_{9 / 2}$ & $1922.05^{\mathrm{A}}$ & $0.032^{\mathrm{A}, \mathrm{B}}$ & $5.85 \times 10^{7 \mathrm{~A}}$ \\
\hline & & $1929.39^{\mathrm{B}}$ & & $5.80 \times 10^{7 \mathrm{~B}}$ \\
\hline $5 f^{2} \mathrm{~F}_{5 / 2}^{o}$ & $12 \mathrm{~d}^{2} \mathrm{D}_{3 / 2}$ & $1931.60^{\mathrm{B}}$ & $0.003^{\mathrm{B}}$ & $5.03 \times 10^{6 \mathrm{~B}}$ \\
\hline $5 \mathrm{f}^{2} \mathrm{~F}_{7 / 2}^{\mathrm{o}}$ & $12 \mathrm{~d}^{2} \mathrm{D}_{5 / 2}$ & $1933.92^{\mathrm{B}}$ & $0.004^{\mathrm{B}}$ & $7.16 \times 10^{6 \mathrm{~B}}$ \\
\hline $7 \mathrm{p}^{2} \mathrm{P}_{1 / 2}^{\mathrm{o}}$ & $12 \mathrm{~d}^{2} \mathrm{D}_{3 / 2}$ & $1961.07^{\mathrm{B}}$ & $0.014^{\mathrm{B}}$ & $2.38 \times 10^{7 \mathrm{~B}}$ \\
\hline $7 \mathrm{p}^{2} \mathrm{P}_{3 / 2}^{\mathrm{o}}$ & $12 \mathrm{~d}^{2} \mathrm{D}_{5 / 2}$ & $2008.77^{\mathrm{B}}$ & $0.024^{\mathrm{B}}$ & $3.99 \times 10^{7 \mathrm{~B}}$ \\
\hline $7 \mathrm{p}^{2} \mathrm{P}_{3 / 2}^{\mathrm{o}}$ & $12 \mathrm{~d}^{2} \mathrm{D}_{3 / 2}$ & $2009.50^{\mathrm{B}}$ & $0.003^{\mathrm{B}}$ & $4.43 \times 10^{6 \mathrm{~B}}$ \\
\hline \multirow[t]{2}{*}{$5 f^{2} F_{5 / 2}^{o}$} & $9 \mathrm{~g}^{2} \mathrm{G}_{7 / 2}$ & $2024.06^{\mathrm{A}}$ & $0.044^{\mathrm{A}, \mathrm{B}}$ & $7.09 \times 10^{7 \mathrm{~A}}$ \\
\hline & & $2009.57^{\mathrm{B}}$ & & $7.26 \times 10^{7 \mathrm{~B}}$ \\
\hline \multirow[t]{2}{*}{$5 f^{2} F_{7 / 2}^{o}$} & $9 g^{2} G_{7 / 2}$ & $2027.35^{\mathrm{A}}$ & $0.002^{\mathrm{A}, \mathrm{B}}$ & $2.61 \times 10^{6 \mathrm{~A}}$ \\
\hline & & $2012.81^{\mathrm{B}}$ & & $2.67 \times 10^{6 \mathrm{~B}}$ \\
\hline \multirow[t]{2}{*}{$5 f^{2} F_{7 / 2}^{o}$} & $9 \mathrm{~g}^{2} \mathrm{G}_{9 / 2}$ & $2027.34^{\mathrm{A}}$ & $0.056^{\mathrm{A}}$ & $9.15 \times 10^{7 \mathrm{~A}}$ \\
\hline & & $2012.81^{\mathrm{B}}$ & $0.057^{\mathrm{B}}$ & $9.36 \times 10^{7 \mathrm{~B}}$ \\
\hline $5 f^{2} F_{5 / 2}^{o}$ & $11 d^{2} D_{3 / 2}$ & $2029.02^{\mathrm{B}}$ & $0.004^{\mathrm{B}}$ & $7.36 \times 10^{6 \mathrm{~B}}$ \\
\hline $5 f^{2} F_{7 / 2}^{o}$ & $11 \mathrm{~d}^{2} \mathrm{D}_{5 / 2}$ & $2031.29^{\mathrm{B}}$ & $0.006^{\mathrm{B}}$ & $1.05 \times 10^{7 \mathrm{~B}}$ \\
\hline $7 \mathrm{p}^{2} \mathrm{P}_{1 / 2}^{\mathrm{o}}$ & $11 \mathrm{~d}^{2} \mathrm{D}_{3 / 2}$ & $2061.56^{\mathrm{B}}$ & $0.022^{\mathrm{B}}$ & $3.47 \times 10^{7 \mathrm{~B}}$ \\
\hline $7 \mathrm{p}^{2} \mathrm{P}_{3 / 2}^{\mathrm{o}}$ & $11 d^{2} D_{5 / 2}$ & $2114.03^{\mathrm{B}}$ & $0.039^{\mathrm{B}}$ & $5.80 \times 10^{7 \mathrm{~B}}$ \\
\hline $7 \mathrm{p}^{2} \mathrm{P}_{3 / 2}^{\mathrm{o}}$ & $11 \mathrm{~d}^{2} \mathrm{D}_{3 / 2}$ & $2115.16^{\mathrm{B}}$ & $0.004^{\mathrm{B}}$ & $6.43 \times 10^{6 B}$ \\
\hline \multirow[t]{2}{*}{$5 \mathrm{f}^{2} \mathrm{~F}_{5 / 2}^{\mathrm{o}}$} & $10 \mathrm{~d}^{2} \mathrm{D}_{5 / 2}$ & $2284.24^{\mathrm{A}}$ & $0.001^{\mathrm{A}, \mathrm{B}}$ & $7.40 \times 10^{5 \mathrm{~A}}$ \\
\hline & & $2182.60^{\mathrm{B}}$ & & $8.22 \times 10^{5 \mathrm{~B}}$ \\
\hline \multirow[t]{2}{*}{$5 f^{2} F_{5 / 2}^{o}$} & $10 \mathrm{~d}^{2} \mathrm{D}_{3 / 2}$ & $2286.23^{\mathrm{A}}$ & $0.008^{\mathrm{A}, \mathrm{B}}$ & $1.03 \times 10^{7 \mathrm{~A}}$ \\
\hline & & $2184.34^{\mathrm{B}}$ & & $1.15 \times 10^{7 \mathrm{~B}}$ \\
\hline \multirow[t]{2}{*}{$5 \mathrm{f}^{2} \mathrm{~F}_{7 / 2}^{\mathrm{o}}$} & $10 \mathrm{~d}^{2} \mathrm{D}_{5 / 2}$ & $2288.43^{\mathrm{A}}$ & $0.012^{\mathrm{A}, \mathrm{B}}$ & $1.47 \times 10^{7 \mathrm{~A}}$ \\
\hline & & $2186.43^{\mathrm{B}}$ & & $1.63 \times 10^{7 \mathrm{~B}}$ \\
\hline \multirow[t]{2}{*}{$7 \mathrm{p}^{2} \mathrm{P}_{1 / 2}^{\mathrm{o}}$} & $10 \mathrm{~d}^{2} \mathrm{D}_{3 / 2}$ & $2327.62^{\mathrm{A}}$ & $0.034^{\mathrm{A}}$ & $4.21 \times 10^{7 \mathrm{~A}}$ \\
\hline & & $2222.09^{\mathrm{B}}$ & $0.040^{\mathrm{B}}$ & $5.41 \times 10^{7 \mathrm{~B}}$ \\
\hline $7 \mathrm{p}^{2} \mathrm{P}_{3 / 2}^{\mathrm{o}}$ & $10 \mathrm{~d}^{2} \mathrm{D}_{5 / 2}$ & $2393.98^{\mathrm{A}}$ & $0.060^{\mathrm{A}}$ & $7.01 \times 10^{7 \mathrm{~A}}$ \\
\hline & & $2282.58^{\mathrm{B}}$ & $0.070^{\mathrm{B}}$ & $8.98 \times 10^{7 \mathrm{~B}}$ \\
\hline $7 \mathrm{p}^{2} \mathrm{P}_{3 / 2}^{\mathrm{o}}$ & $10 \mathrm{~d}^{2} \mathrm{D}_{3 / 2}$ & $2396.17^{\mathrm{A}}$ & $0.007^{\mathrm{A}}$ & $7.73 \times 10^{6 \mathrm{~A}}$ \\
\hline
\end{tabular}


Table 2: Continued.

\begin{tabular}{|c|c|c|c|c|}
\hline Lower level & Upper level & $\lambda$ & $g f$ & $g A_{k i}$ \\
\hline & & $2284.49^{\mathrm{B}}$ & $0.008^{\mathrm{B}}$ & $9.95 \times 10^{6 \mathrm{~B}}$ \\
\hline $7 \mathrm{~d}^{2} \mathrm{D}_{3 / 2}$ & $12 \mathrm{f}^{2} \mathrm{~F}_{5 / 2}^{\mathrm{o}}$ & $2786.97^{\mathrm{B}}$ & $0.045^{\mathrm{B}}$ & $3.86 \times 10^{7 \mathrm{~B}}$ \\
\hline $7 d^{2} D_{5 / 2}$ & $12 \mathrm{f}^{2} \mathrm{~F}_{7 / 2}^{\mathrm{o}}$ & $2802.54^{\mathrm{B}}$ & $0.064^{\mathrm{B}}$ & $5.42 \times 10^{7 \mathrm{~B}}$ \\
\hline $7 \mathrm{~d}^{2} \mathrm{D}_{5 / 2}$ & $12 \mathrm{f}^{2} \mathrm{~F}_{5 / 2}^{\mathrm{o}}$ & $2802.90^{\mathrm{B}}$ & $0.003^{\mathrm{B}}$ & $2.71 \times 10^{6 \mathrm{~B}}$ \\
\hline $7 d^{2} D_{3 / 2}$ & $11 f^{2} \mathrm{~F}_{5 / 2}^{\mathrm{o}}$ & $2925.08^{\mathrm{B}}$ & $0.064^{\mathrm{B}}$ & $4.98 \times 10^{7 \mathrm{~B}}$ \\
\hline $7 d^{2} D_{5 / 2}$ & $11 \mathrm{f}^{2} \mathrm{~F}_{7 / 2}^{\mathrm{o}}$ & $2942.09^{\mathrm{B}}$ & $0.091^{\mathrm{B}}$ & $7.00 \times 10^{7 \mathrm{~B}}$ \\
\hline $7 d^{2} D_{5 / 2}$ & $11 \mathrm{f}^{2} \mathrm{~F}_{5 / 2}^{\mathrm{o}}$ & $2942.64^{\mathrm{B}}$ & $0.004^{\mathrm{B}}$ & $3.50 \times 10^{6 \mathrm{~B}}$ \\
\hline $7 d^{2} D_{3 / 2}$ & $12 \mathrm{p}^{2} \mathrm{P}_{3 / 2}^{\mathrm{o}}$ & $3116.57^{\mathrm{B}}$ & $0.001^{\mathrm{B}}$ & $4.10 \times 10^{5 \mathrm{~B}}$ \\
\hline $7 \mathrm{~d}^{2} \mathrm{D}_{3 / 2}$ & $12 \mathrm{p}^{2} \mathrm{P}_{1 / 2}^{\mathrm{o}}$ & $3125.35^{\mathrm{B}}$ & $0.003^{\mathrm{B}}$ & $2.03 \times 10^{6 \mathrm{~B}}$ \\
\hline \multirow[t]{2}{*}{$7 \mathrm{~d}^{2} \mathrm{D}_{3 / 2}$} & $10 \mathrm{f}^{2} \mathrm{~F}_{5 / 2}^{\mathrm{o}}$ & $3135.24^{\mathrm{A}, \mathrm{B}}$ & $0.103^{\mathrm{A}}$ & $6.99 \times 10^{7 \mathrm{~A}}$ \\
\hline & & & $0.096^{\mathrm{B}}$ & $6.53 \times 10^{7 \mathrm{~B}}$ \\
\hline $7 d^{2} D_{5 / 2}$ & $12 \mathrm{p}^{2} \mathrm{P}_{3 / 2}^{\mathrm{o}}$ & $3136.51^{\mathrm{B}}$ & $0.005^{\mathrm{B}}$ & $3.62 \times 10^{6 \mathrm{~B}}$ \\
\hline \multirow{2}{*}{$7 d^{2} D_{5 / 2}$} & $10 \mathrm{f}^{2} \mathrm{~F}_{7 / 2}^{\mathrm{o}}$ & $3154.57^{\mathrm{A}}$ & $0.146^{\mathrm{A}}$ & $9.78 \times 10^{7 \mathrm{~A}}$ \\
\hline & & $3154.55^{\mathrm{B}}$ & $0.137^{\mathrm{B}}$ & $9.16 \times 10^{7 \mathrm{~B}}$ \\
\hline \multirow[t]{2}{*}{$7 \mathrm{~d}^{2} \mathrm{D}_{5 / 2}$} & $10 f^{2} \mathrm{~F}_{5 / 2}^{\mathrm{o}}$ & $3155.45^{\mathrm{A}}$ & $0.007^{\mathrm{A}, \mathrm{B}}$ & $4.89 \times 10^{6 \mathrm{~A}}$ \\
\hline & & $3155.42^{\mathrm{B}}$ & & $4.58 \times 10^{6 \mathrm{~B}}$ \\
\hline \multirow[t]{2}{*}{$6 f^{2} F_{5 / 2}^{o}$} & $10 g^{2} G_{7 / 2}$ & $3392.75^{\mathrm{A}}$ & $0.098^{\mathrm{A}}$ & $5.70 \times 10^{7 \mathrm{~A}}$ \\
\hline & & $3370.11^{\mathrm{B}}$ & $0.099^{\mathrm{B}}$ & $5.82 \times 10^{7 \mathrm{~B}}$ \\
\hline \multirow[t]{2}{*}{$6 f^{2} F_{7 / 2}^{o}$} & $10 \operatorname{g~}^{2} \mathrm{G}_{7 / 2}$ & $3398.40^{\mathrm{A}}$ & $0.004^{\mathrm{A}, \mathrm{B}}$ & $2.10 \times 10^{6 \mathrm{~A}}$ \\
\hline & & $3375.69^{\mathrm{B}}$ & & $2.14 \times 10^{6 \mathrm{~B}}$ \\
\hline \multirow{2}{*}{$6 \mathrm{f}^{2} \mathrm{~F}_{7 / 2}^{\mathrm{o}}$} & $10 g^{2} \mathrm{G}_{9 / 2}$ & $3398.41^{\mathrm{A}}$ & $0.127^{\mathrm{A}}$ & $7.35 \times 10^{7 \mathrm{~A}}$ \\
\hline & & $3375.69^{\mathrm{B}}$ & $0.128^{\mathrm{B}}$ & $7.50 \times 10^{7 \mathrm{~B}}$ \\
\hline $6 \mathrm{f}^{2} \mathrm{~F}_{5 / 2}^{\mathrm{o}}$ & $12 \mathrm{~d}^{2} \mathrm{D}_{5 / 2}$ & $3406.76^{\mathrm{B}}$ & $0.001^{\mathrm{B}}$ & $4.58 \times 10^{5 \mathrm{~B}}$ \\
\hline $6 \mathrm{f}^{2} \mathrm{~F}_{5 / 2}^{\mathrm{o}}$ & $12 \mathrm{~d}^{2} \mathrm{D}_{3 / 2}$ & $3408.88^{\mathrm{B}}$ & $0.011^{\mathrm{B}}$ & $6.40 \times 10^{6 \mathrm{~B}}$ \\
\hline $6 \mathrm{f}^{2} \mathrm{~F}_{7 / 2}^{\mathrm{o}}$ & $12 \mathrm{~d}^{2} \mathrm{D}_{5 / 2}$ & $3412.46^{\mathrm{B}}$ & $0.016^{\mathrm{B}}$ & $9.11 \times 10^{6 \mathrm{~B}}$ \\
\hline $7 \mathrm{~d}^{2} \mathrm{D}_{3 / 2}$ & $11 p^{2} \mathrm{P}_{3 / 2}^{\mathrm{o}}$ & $3452.08^{\mathrm{B}}$ & $0.001^{\mathrm{B}}$ & $5.78 \times 10^{5 \mathrm{~B}}$ \\
\hline $7 \mathrm{~d}^{2} \mathrm{D}_{3 / 2}$ & $11 \mathrm{p}^{2} \mathrm{P}_{1 / 2}^{\mathrm{o}}$ & $3467.38^{\mathrm{B}}$ & $0.005^{\mathrm{B}}$ & $2.85 \times 10^{6 \mathrm{~B}}$ \\
\hline $7 d^{2} D_{5 / 2}$ & $11 \mathrm{p}^{2} \mathrm{P}_{3 / 2}^{\mathrm{o}}$ & $3476.57^{\mathrm{B}}$ & $0.009^{B}$ & $5.09 \times 10^{6 \mathrm{~B}}$ \\
\hline $8 \mathrm{p}^{2} \mathrm{P}_{1 / 2}^{\mathrm{o}}$ & $12 \mathrm{~d}^{2} \mathrm{D}_{3 / 2}$ & $3493.71^{\mathrm{B}}$ & $0.028^{\mathrm{B}}$ & $1.54 \times 10^{7 \mathrm{~B}}$ \\
\hline $8 \mathrm{p}^{2} \mathrm{P}_{3 / 2}^{\mathrm{o}}$ & $12 \mathrm{~d}^{2} \mathrm{D}_{5 / 2}$ & $3569.21^{\mathrm{B}}$ & $0.050^{\mathrm{B}}$ & $2.60 \times 10^{7 \mathrm{~B}}$ \\
\hline $8 \mathrm{p}^{2} \mathrm{P}_{3 / 2}^{\mathrm{o}}$ & $12 \mathrm{~d}^{2} \mathrm{D}_{3 / 2}$ & $3571.54^{\mathrm{B}}$ & $0.005^{\mathrm{B}}$ & $2.88 \times 10^{6 \mathrm{~B}}$ \\
\hline \multirow[t]{2}{*}{$6 \mathrm{f}^{2} \mathrm{~F}_{5 / 2}^{\mathrm{o}}$} & $9 \mathrm{~g}^{2} \mathrm{G}_{7 / 2}$ & $3707.80^{\mathrm{A}}$ & $0.181^{\mathrm{A}}$ & $8.76 \times 10^{7 \mathrm{~A}}$ \\
\hline & & $3659.44^{\mathrm{B}}$ & $0.183^{\mathrm{B}}$ & $9.13 \times 10^{7 \mathrm{~B}}$ \\
\hline \multirow[t]{2}{*}{$6 \mathrm{f}^{2} \mathrm{~F}_{7 / 2}^{\mathrm{o}}$} & $9 \mathrm{~g}^{2} \mathrm{G}_{7 / 2}$ & $3714.55^{\mathrm{A}}$ & $0.007^{\mathrm{A}, \mathrm{B}}$ & $3.23 \times 10^{6 \mathrm{~A}}$ \\
\hline & & $3666.01^{\mathrm{B}}$ & & $3.36 \times 10^{6 \mathrm{~B}}$ \\
\hline \multirow[t]{2}{*}{$6 f^{2} \mathrm{~F}_{7 / 2}^{\mathrm{o}}$} & $9 g^{2} G_{9 / 2}$ & $3714.49^{\mathrm{A}}$ & $0.234^{\mathrm{A}}$ & $1.13 \times 10^{8 \mathrm{~A}}$ \\
\hline & & $3666.01^{\mathrm{B}}$ & $0.237^{\mathrm{B}}$ & $1.18 \times 10^{8 \mathrm{~B}}$ \\
\hline $6 \mathrm{f}^{2} \mathrm{~F}_{5 / 2}^{\mathrm{o}}$ & $11 \mathrm{~d}^{2} \mathrm{D}_{5 / 2}$ & $3720.97^{\mathrm{B}}$ & $0.001^{\mathrm{B}}$ & $6.83 \times 10^{5 \mathrm{~B}}$ \\
\hline $6 \mathrm{f}^{2} \mathrm{~F}_{5 / 2}^{\mathrm{o}}$ & $11 \mathrm{~d}^{2} \mathrm{D}_{3 / 2}$ & $3724.47^{\mathrm{B}}$ & $0.020^{\mathrm{B}}$ & $9.54 \times 10^{6 \mathrm{~B}}$ \\
\hline $6 \mathrm{f}^{2} \mathrm{~F}_{7 / 2}^{\mathrm{o}}$ & $11 \mathrm{~d}^{2} \mathrm{D}_{5 / 2}$ & $3727.77^{\mathrm{B}}$ & $0.028^{\mathrm{B}}$ & $1.36 \times 10^{7 \mathrm{~B}}$ \\
\hline $8 \mathrm{p}^{2} \mathrm{P}_{1 / 2}^{\mathrm{o}}$ & $11 \mathrm{~d}^{2} \mathrm{D}_{3 / 2}$ & $3825.97^{\mathrm{B}}$ & $0.050^{\mathrm{B}}$ & $2.30 \times 10^{7 \mathrm{~B}}$ \\
\hline $8 \mathrm{p}^{2} \mathrm{P}_{3 / 2}^{\mathrm{o}}$ & $11 \mathrm{~d}^{2} \mathrm{D}_{5 / 2}$ & $3915.63^{\mathrm{B}}$ & $0.089^{\mathrm{B}}$ & $3.86 \times 10^{7 \mathrm{~B}}$ \\
\hline $8 \mathrm{p}^{2} \mathrm{P}_{3 / 2}^{\mathrm{o}}$ & $11 \mathrm{~d}^{2} \mathrm{D}_{3 / 2}$ & $3919.50^{\mathrm{B}}$ & $0.010^{\mathrm{B}}$ & $4.28 \times 10^{6 \mathrm{~B}}$ \\
\hline \multirow[t]{2}{*}{$7 \mathrm{~d}^{2} \mathrm{D}_{3 / 2}$} & $10 \mathrm{p}^{2} \mathrm{P}_{3 / 2}^{\mathrm{o}}$ & $4093.95^{\mathrm{A}}$ & $0.003^{\mathrm{A}}$ & $1.05 \times 10^{6 \mathrm{~A}}$ \\
\hline & & $4093.09^{\mathrm{B}}$ & $0.002^{\mathrm{B}}$ & $8.64 \times 10^{5 \mathrm{~B}}$ \\
\hline \multirow[t]{2}{*}{$7 d^{2} D_{3 / 2}$} & $10 p^{2} \mathrm{P}_{1 / 2}^{\mathrm{o}}$ & $4126.00^{\mathrm{A}}$ & $0.013^{\mathrm{A}}$ & $5.11 \times 10^{6 \mathrm{~A}}$ \\
\hline & & $4125.12^{\mathrm{B}}$ & $0.011^{\mathrm{B}}$ & $4.22 \times 10^{6 \mathrm{~B}}$ \\
\hline \multirow[t]{2}{*}{$7 \mathrm{~d}^{2} \mathrm{D}_{5 / 2}$} & $10 \mathrm{p}^{2} \mathrm{P}_{3 / 2}^{\mathrm{o}}$ & $4128.47^{\mathrm{A}}$ & $0.023^{\mathrm{A}}$ & $9.13 \times 10^{6 \mathrm{~A}}$ \\
\hline & & $4127.55^{\mathrm{B}}$ & $0.019^{\mathrm{B}}$ & $7.59 \times 10^{6 \mathrm{~B}}$ \\
\hline \multirow[t]{2}{*}{$6 f^{2} \mathrm{~F}_{5 / 2}^{\mathrm{o}}$} & $10 \mathrm{~d}^{2} \mathrm{D}_{5 / 2}$ & $4685.40^{\mathrm{A}}$ & $0.003^{\mathrm{A}, \mathrm{B}}$ & $8.22 \times 10^{5 \mathrm{~A}}$ \\
\hline & & $4276.87^{\mathrm{B}}$ & & $1.10 \times 10^{6 \mathrm{~B}}$ \\
\hline $6 \mathrm{f}^{2} \mathrm{~F}_{5 / 2}^{\mathrm{o}}$ & $10 \mathrm{~d}^{2} \mathrm{D}_{3 / 2}$ & $4693.78^{\mathrm{A}}$ & $0.038^{\mathrm{A}}$ & $1.14 \times 10^{7 \mathrm{~A}}$ \\
\hline
\end{tabular}


Table 2: Continued.

\begin{tabular}{|c|c|c|c|c|}
\hline \multirow[b]{2}{*}{ Lower level } & Transition & \multirow{2}{*}{$\lambda$} & \multirow{2}{*}{$g f$} & \multirow{2}{*}{$g A_{k i}$} \\
\hline & Upper level & & & \\
\hline & & $4283.55^{\mathrm{B}}$ & $0.042^{\mathrm{B}}$ & $1.54 \times 10^{7 \mathrm{~B}}$ \\
\hline \multirow[t]{2}{*}{$6 \mathrm{f}^{2} \mathrm{~F}_{7 / 2}^{\mathrm{o}}$} & $10 \mathrm{~d}^{2} \mathrm{D}_{5 / 2}$ & $4696.17^{\mathrm{A}}$ & $0.054^{\mathrm{A}}$ & $1.63 \times 10^{7 \mathrm{~A}}$ \\
\hline & & $4285.85^{\mathrm{B}}$ & $0.060^{\mathrm{B}}$ & $2.19 \times 10^{7 \mathrm{~B}}$ \\
\hline \multirow[t]{2}{*}{$8 p^{2} P_{1 / 2}^{o}$} & $10 \mathrm{~d}^{2} \mathrm{D}_{3 / 2}$ & $4856.14^{\mathrm{A}}$ & $0.091^{\mathrm{A}}$ & $2.57 \times 10^{7 \mathrm{~A}}$ \\
\hline & & $4418.37^{\mathrm{B}}$ & $0.110^{\mathrm{B}}$ & $3.74 \times 10^{7 \mathrm{~B}}$ \\
\hline \multirow[t]{2}{*}{$8 \mathrm{p}^{2} \mathrm{P}_{3 / 2}^{\mathrm{o}}$} & $10 \mathrm{~d}^{2} \mathrm{D}_{5 / 2}$ & $4998.26^{\mathrm{A}}$ & $0.160^{\mathrm{A}}$ & $4.26 \times 10^{7 \mathrm{~A}}$ \\
\hline & & $4536.05^{\mathrm{B}}$ & $0.192^{\mathrm{B}}$ & $6.22 \times 10^{7 \mathrm{~B}}$ \\
\hline \multirow[t]{2}{*}{$8 \mathrm{p}^{2} \mathrm{P}_{3 / 2}^{\mathrm{o}}$} & $10 \mathrm{~d}^{2} \mathrm{D}_{3 / 2}$ & $5007.81^{\mathrm{A}}$ & $0.018^{\mathrm{A}}$ & $4.69 \times 10^{6 \mathrm{~A}}$ \\
\hline & & $4543.57^{\mathrm{B}}$ & $0.021^{\mathrm{B}}$ & $6.88 \times 10^{6 \mathrm{~B}}$ \\
\hline $8 \mathrm{~d}^{2} \mathrm{D}_{3 / 2}$ & $12 \mathrm{f}^{2} \mathrm{~F}_{5 / 2}^{\mathrm{o}}$ & $4613.96^{\mathrm{B}}$ & $0.058^{\mathrm{B}}$ & $1.82 \times 10^{7 \mathrm{~B}}$ \\
\hline $8 d^{2} D_{5 / 2}$ & $12 \mathrm{f}^{2} \mathrm{~F}_{7 / 2}^{\mathrm{o}}$ & $4637.33^{\mathrm{B}}$ & $0.083^{\mathrm{B}}$ & $2.57 \times 10^{7 \mathrm{~B}}$ \\
\hline $8 d^{2} D_{5 / 2}$ & $12 \mathrm{f}^{2} \mathrm{~F}_{5 / 2}^{\mathrm{o}}$ & $4638.30^{\mathrm{B}}$ & $0.004^{\mathrm{B}}$ & $1.28 \times 10^{6 \mathrm{~B}}$ \\
\hline $8 \mathrm{~d}^{2} \mathrm{D}_{3 / 2}$ & $11 \mathrm{f}^{2} \mathrm{~F}_{5 / 2}^{\mathrm{o}}$ & $5005.22^{\mathrm{B}}$ & $0.086^{\mathrm{B}}$ & $2.30 \times 10^{7 \mathrm{~B}}$ \\
\hline $8 d^{2} D_{5 / 2}$ & $11 \mathrm{f}^{2} \mathrm{~F}_{7 / 2}^{\mathrm{o}}$ & $5032.28^{\mathrm{B}}$ & $0.123^{\mathrm{B}}$ & $3.23 \times 10^{7 \mathrm{~B}}$ \\
\hline $8 d^{2} D_{5 / 2}$ & $11 \mathrm{f}^{2} \mathrm{~F}_{5 / 2}^{\mathrm{o}}$ & $5033.88^{\mathrm{B}}$ & $0.006^{\mathrm{B}}$ & $1.61 \times 10^{6 \mathrm{~B}}$ \\
\hline $6 \mathrm{~g}^{2} \mathrm{G}_{9 / 2}$ & $12 \mathrm{f}^{2} \mathrm{~F}_{7 / 2}^{\mathrm{o}}$ & $5136.94^{\mathrm{B}}$ & $0.001^{\mathrm{B}}$ & $1.75 \times 10^{5 \mathrm{~B}}$ \\
\hline $6 \mathrm{~g}^{2} \mathrm{G}_{7 / 2}$ & $12 \mathrm{f}^{2} \mathrm{~F}_{5 / 2}^{\mathrm{o}}$ & $5137.90^{\mathrm{B}}$ & $0.001^{\mathrm{B}}$ & $1.35 \times 10^{5 \mathrm{~B}}$ \\
\hline $8 \mathrm{~d}^{2} \mathrm{D}_{3 / 2}$ & $12 \mathrm{p}^{2} \mathrm{P}_{3 / 2}^{\mathrm{o}}$ & $5593.28^{\mathrm{B}}$ & $0.001^{\mathrm{B}}$ & $1.96 \times 10^{5 \mathrm{~B}}$ \\
\hline $8 \mathrm{~d}^{2} \mathrm{D}_{3 / 2}$ & $12 \mathrm{p}^{2} \mathrm{P}_{1 / 2}^{\mathrm{o}}$ & $5621.62^{\mathrm{B}}$ & $0.005^{\mathrm{B}}$ & $9.65 \times 10^{5 \mathrm{~B}}$ \\
\hline $6 \mathrm{~g}^{2} \mathrm{G}_{9 / 2}$ & $11 \mathrm{f}^{2} \mathrm{~F}_{7 / 2}^{\mathrm{o}}$ & $5626.07^{\mathrm{B}}$ & $0.001^{\mathrm{B}}$ & $2.71 \times 10^{5 \mathrm{~B}}$ \\
\hline $6 \mathrm{~g}^{2} \mathrm{G}_{7 / 2}$ & $11 \mathrm{f}^{2} \mathrm{~F}_{5 / 2}^{\mathrm{o}}$ & $5627.78^{\mathrm{B}}$ & $0.001^{\mathrm{B}}$ & $2.09 \times 10^{5 B}$ \\
\hline $8 d^{2} D_{5 / 2}$ & $12 \mathrm{p}^{2} \mathrm{P}_{3 / 2}^{\mathrm{o}}$ & $5629.09^{\mathrm{B}}$ & $0.008^{B}$ & $1.73 \times 10^{6 \mathrm{~B}}$ \\
\hline \multirow[t]{2}{*}{$8 \mathrm{~d}^{2} \mathrm{D}_{3 / 2}$} & $10 \mathrm{f}^{2} \mathrm{~F}_{5 / 2}^{\mathrm{o}}$ & $5653.76^{\mathrm{A}}$ & $0.144^{\mathrm{A}}$ & $3.02 \times 10^{7 \mathrm{~A}}$ \\
\hline & & $5653.69^{\mathrm{B}}$ & $0.138^{\mathrm{B}}$ & $2.87 \times 10^{7 \mathrm{~B}}$ \\
\hline \multirow[t]{2}{*}{$8 d^{2} D_{5 / 2}$} & $10 \mathrm{f}^{2} \mathrm{~F}_{7 / 2}^{\mathrm{o}}$ & $5687.53^{\mathrm{A}}$ & $0.203^{\mathrm{A}}$ & $4.23 \times 10^{7 \mathrm{~A}}$ \\
\hline & & $5687.46^{\mathrm{B}}$ & $0.196^{\mathrm{B}}$ & $4.03 \times 10^{7 \mathrm{~B}}$ \\
\hline \multirow[t]{2}{*}{$8 d^{2} D_{5 / 2}$} & $10 \mathrm{f}^{2} \mathrm{~F}_{5 / 2}^{\mathrm{o}}$ & $5690.36^{\mathrm{A}}$ & $0.010^{\mathrm{A}, \mathrm{B}}$ & $2.11 \times 10^{6 \mathrm{~A}}$ \\
\hline & & $5690.29^{\mathrm{B}}$ & & $2.01 \times 10^{6 \mathrm{~B}}$ \\
\hline \multirow[t]{2}{*}{$7 \mathrm{f}^{2} \mathrm{~F}_{5 / 2}^{\mathrm{o}}$} & $10 \mathrm{~g}^{2} \mathrm{G}_{7 / 2}$ & $5773.05^{\mathrm{A}}$ & $0.247^{\mathrm{A}}$ & $4.95 \times 10^{7 \mathrm{~A}}$ \\
\hline & & $5707.79^{\mathrm{B}}$ & $0.250^{\mathrm{B}}$ & $5.13 \times 10^{7 \mathrm{~B}}$ \\
\hline \multirow[t]{2}{*}{$7 f^{2} F_{7 / 2}^{o}$} & $10 \operatorname{g~}^{2} G_{7 / 2}$ & $5784.03^{\mathrm{A}}$ & $0.009^{\mathrm{A}, \mathrm{B}}$ & $1.82 \times 10^{6 \mathrm{~A}}$ \\
\hline & & $5718.53^{\mathrm{B}}$ & & $1.89 \times 10^{6 \mathrm{~B}}$ \\
\hline \multirow[t]{2}{*}{$7 f^{2} F_{7 / 2}^{o}$} & $10 \mathrm{~g}^{2} \mathrm{G}_{9 / 2}$ & $5784.05^{\mathrm{A}}$ & $0.320^{\mathrm{A}}$ & $6.38 \times 10^{7 \mathrm{~A}}$ \\
\hline & & $5718.53^{\mathrm{B}}$ & $0.324^{\mathrm{B}}$ & $6.61 \times 10^{7 \mathrm{~B}}$ \\
\hline $7 f^{2} F_{5 / 2}^{o}$ & $12 \mathrm{~d}^{2} \mathrm{D}_{5 / 2}$ & $5813.72^{\mathrm{B}}$ & $0.002^{\mathrm{B}}$ & $4.69 \times 10^{5 \mathrm{~B}}$ \\
\hline $7 f^{2} F_{5 / 2}^{o}$ & $12 \mathrm{~d}^{2} \mathrm{D}_{3 / 2}$ & $5819.89^{\mathrm{B}}$ & $0.033^{\mathrm{B}}$ & $6.55 \times 10^{6 \mathrm{~B}}$ \\
\hline $7 \mathrm{f}^{2} \mathrm{~F}_{7 / 2}^{\mathrm{o}}$ & $12 \mathrm{~d}^{2} \mathrm{D}_{5 / 2}$ & $5824.86^{\mathrm{B}}$ & $0.047^{\mathrm{B}}$ & $9.33 \times 10^{6 \mathrm{~B}}$ \\
\hline $9 \mathrm{p}^{2} \mathrm{P}_{1 / 2}^{\mathrm{o}}$ & $12 \mathrm{~d}^{2} \mathrm{D}_{3 / 2}$ & $5996.59^{\mathrm{B}}$ & $0.061^{\mathrm{B}}$ & $1.12 \times 10^{7 \mathrm{~B}}$ \\
\hline $9 \mathrm{p}^{2} \mathrm{P}_{3 / 2}^{\mathrm{o}}$ & $12 \mathrm{~d}^{2} \mathrm{D}_{5 / 2}$ & $6131.85^{\mathrm{B}}$ & $0.107^{\mathrm{B}}$ & $1.89 \times 10^{7 \mathrm{~B}}$ \\
\hline $9 \mathrm{p}^{2} \mathrm{P}_{3 / 2}^{\mathrm{o}}$ & $12 \mathrm{~d}^{2} \mathrm{D}_{3 / 2}$ & $6138.72^{\mathrm{B}}$ & $0.012^{\mathrm{B}}$ & $2.10 \times 10^{6 \mathrm{~B}}$ \\
\hline \multirow[t]{2}{*}{$6 g^{2} \mathrm{G}_{9 / 2}$} & $10 \mathrm{f}^{2} \mathrm{~F}_{7 / 2}^{\mathrm{o}}$ & $6457.77^{\mathrm{A}}$ & $0.003^{\mathrm{A}, \mathrm{B}}$ & $4.51 \times 10^{5 \mathrm{~A}}$ \\
\hline & & $6457.76^{\mathrm{B}}$ & & $4.59 \times 10^{5 \mathrm{~B}}$ \\
\hline \multirow[t]{2}{*}{$6 \mathrm{~g}^{2} \mathrm{G}_{7 / 2}$} & $10 \mathrm{f}^{2} \mathrm{~F}_{5 / 2}^{\mathrm{o}}$ & $6461.09^{\mathrm{A}}$ & $0.002^{\mathrm{A}, \mathrm{B}}$ & $3.48 \times 10^{5 \mathrm{~A}}$ \\
\hline & & $6461.03^{\mathrm{B}}$ & & $3.53 \times 10^{5 \mathrm{~B}}$ \\
\hline \multirow[t]{2}{*}{$7 f^{2} F_{5 / 2}^{o}$} & $9 \mathrm{~g}^{2} \mathrm{G}_{7 / 2}$ & $6748.79^{\mathrm{A}}$ & $0.512^{\mathrm{A}}$ & $7.50 \times 10^{7 \mathrm{~A}}$ \\
\hline & & $6590.26^{\mathrm{B}}$ & $0.525^{\mathrm{B}}$ & $8.07 \times 10^{7 \mathrm{~B}}$ \\
\hline \multirow[t]{2}{*}{$7 \mathrm{f}^{2} \mathrm{~F}_{7 / 2}^{\mathrm{o}}$} & $9 \mathrm{~g}^{2} \mathrm{G}_{7 / 2}$ & $6763.80^{\mathrm{A}}$ & $0.019^{\mathrm{A}, \mathrm{B}}$ & $2.76 \times 10^{6 \mathrm{~A}}$ \\
\hline & & $6604.58^{\mathrm{B}}$ & & $2.97 \times 10^{6 B}$ \\
\hline \multirow[t]{2}{*}{$7 \mathrm{f}^{2} \mathrm{~F}_{7 / 2}^{\mathrm{o}}$} & $9 \mathrm{~g}^{2} \mathrm{G}_{9 / 2}$ & $6763.63^{\mathrm{A}}$ & $0.663^{\mathrm{A}}$ & $9.66 \times 10^{7 \mathrm{~A}}$ \\
\hline & & $6604.58^{\mathrm{B}}$ & $0.679^{\mathrm{B}}$ & $1.04 \times 10^{8 \mathrm{~B}}$ \\
\hline
\end{tabular}


TABle 2: Continued.

\begin{tabular}{|c|c|c|c|c|}
\hline \multirow[b]{2}{*}{ Lower level } & Transition & \multirow{2}{*}{$\lambda$} & \multirow{2}{*}{$g f$} & \multirow{2}{*}{$g A_{k i}$} \\
\hline & Upper level & & & \\
\hline $8 d^{2} D_{3 / 2}$ & $11 \mathrm{p}^{2} \mathrm{P}_{3 / 2}^{\mathrm{o}}$ & $6775.04^{\mathrm{B}}$ & $0.002^{\mathrm{B}}$ & $2.86 \times 10^{5 \mathrm{~B}}$ \\
\hline $7 \mathrm{f}^{2} \mathrm{~F}_{5 / 2}^{\mathrm{o}}$ & $11 \mathrm{~d}^{2} \mathrm{D}_{5 / 2}$ & $6792.55^{\mathrm{B}}$ & $0.005^{\mathrm{B}}$ & $7.23 \times 10^{5 \mathrm{~B}}$ \\
\hline $7 \mathrm{f}^{2} \mathrm{~F}_{5 / 2}^{\mathrm{o}}$ & $11 \mathrm{~d}^{2} \mathrm{D}_{3 / 2}$ & $6804.23^{\mathrm{B}}$ & $0.070^{\mathrm{B}}$ & $1.01 \times 10^{7 \mathrm{~B}}$ \\
\hline $7 f^{2} \mathrm{~F}_{7 / 2}^{\mathrm{o}}$ & $11 d^{2} D_{5 / 2}$ & $6807.77^{\mathrm{B}}$ & $0.100^{\mathrm{B}}$ & $1.44 \times 10^{7 \mathrm{~B}}$ \\
\hline $8 d^{2} D_{5 / 2}$ & $11 \mathrm{p}^{2} \mathrm{P}_{3 / 2}^{\mathrm{o}}$ & $6827.66^{\mathrm{B}}$ & $0.017^{\mathrm{B}}$ & $2.52 \times 10^{6 \mathrm{~B}}$ \\
\hline $8 \mathrm{~d}^{2} \mathrm{D}_{3 / 2}$ & $11 \mathrm{p}^{2} \mathrm{P}_{1 / 2}^{\mathrm{o}}$ & $6834.22^{\mathrm{B}}$ & $0.010^{\mathrm{B}}$ & $1.39 \times 10^{6 \mathrm{~B}}$ \\
\hline $9 \mathrm{p}^{2} \mathrm{P}_{1 / 2}^{\mathrm{o}}$ & $11 \mathrm{~d}^{2} \mathrm{D}_{3 / 2}$ & $7046.99^{\mathrm{B}}$ & $0.130^{\mathrm{B}}$ & $1.75 \times 10^{7 \mathrm{~B}}$ \\
\hline $9 \mathrm{p}^{2} \mathrm{P}_{3 / 2}^{\mathrm{o}}$ & $11 \mathrm{~d}^{2} \mathrm{D}_{5 / 2}$ & $7230.87^{\mathrm{B}}$ & $0.228^{\mathrm{B}}$ & $2.91 \times 10^{7 \mathrm{~B}}$ \\
\hline $9 \mathrm{p}^{2} \mathrm{P}_{3 / 2}^{\mathrm{o}}$ & $11 \mathrm{~d}^{2} \mathrm{D}_{3 / 2}$ & $7244.09^{\mathrm{B}}$ & $0.025^{\mathrm{B}}$ & $3.21 \times 10^{6 \mathrm{~B}}$ \\
\hline $9 d^{2} D_{3 / 2}$ & $12 \mathrm{f}^{2} \mathrm{~F}_{5 / 2}^{\mathrm{o}}$ & $7457.70^{\mathrm{B}}$ & $0.079^{\mathrm{B}}$ & $9.47 \times 10^{6 \mathrm{~B}}$ \\
\hline $9 d^{2} D_{5 / 2}$ & $12 \mathrm{f}^{2} \mathrm{~F}_{7 / 2}^{\mathrm{o}}$ & $7494.42^{\mathrm{B}}$ & $0.112^{\mathrm{B}}$ & $1.33 \times 10^{7 \mathrm{~B}}$ \\
\hline $9 d^{2} D_{5 / 2}$ & $12 \mathrm{f}^{2} \mathrm{~F}_{5 / 2}^{\mathrm{o}}$ & $7496.98^{\mathrm{B}}$ & $0.006^{\mathrm{B}}$ & $6.66 \times 10^{5 \mathrm{~B}}$ \\
\hline $7 \mathrm{~g}^{2} \mathrm{G}_{9 / 2}$ & $12 \mathrm{f}^{2} \mathrm{~F}_{7 / 2}^{\mathrm{o}}$ & $8263.95^{\mathrm{B}}$ & $0.003^{\mathrm{B}}$ & $2.86 \times 10^{5 \mathrm{~B}}$ \\
\hline $7 \mathrm{~g}^{2} \mathrm{G}_{7 / 2}$ & $12 \mathrm{f}^{2} \mathrm{~F}_{5 / 2}^{\mathrm{o}}$ & $8266.14^{\mathrm{B}}$ & $0.002^{\mathrm{B}}$ & $2.21 \times 10^{5 \mathrm{~B}}$ \\
\hline $9 \mathrm{~d}^{2} \mathrm{D}_{3 / 2}$ & $11 \mathrm{f}^{2} \mathrm{~F}_{5 / 2}^{\mathrm{o}}$ & $8536.25^{\mathrm{B}}$ & $0.124^{\mathrm{B}}$ & $1.14 \times 10^{7 \mathrm{~B}}$ \\
\hline $9 d^{2} D_{5 / 2}$ & $11 \mathrm{f}^{2} \mathrm{~F}_{7 / 2}^{\mathrm{o}}$ & $8583.10^{\mathrm{B}}$ & $0.176^{\mathrm{B}}$ & $1.60 \times 10^{7 \mathrm{~B}}$ \\
\hline $9 d^{2} D_{5 / 2}$ & $11 f^{2} \mathrm{~F}_{5 / 2}^{\mathrm{o}}$ & $8587.74^{\mathrm{B}}$ & $0.009^{\mathrm{B}}$ & $7.97 \times 10^{5 \mathrm{~B}}$ \\
\hline
\end{tabular}

between electrons $i$ and $j$ averaged over all possible magnetic quantum numbers.

In this method, relativistic corrections have been limited to calculations to the mass-velocity and the Darwin corrections by using the relativistic correction to total binding energy. The total binding energy can be given in by formulas (7.57), (7.58), and (7.59) in [16].

\section{Results and Discussion}

We calculated the radiative parameters (wavelengths, oscillator strengths, and transition probabilities) for electric dipole (E1) transitions in La III $(Z=57)$ using HFR code [15]. We have taken into account $5 \mathrm{p}^{6} \mathrm{nd}, 5 \mathrm{p}^{6} \mathrm{ng}(n=5-10), 5 \mathrm{p}^{6} \mathrm{~ns}(n=$ 6-10), 5 $p^{5} 6 s 6 p, 5 p^{5} 6 s 4 f, 5 p^{5} 5 d 6 p, 5 p^{6}$ nf $(n=4-10), 5 p^{6} n p$ $(n=6-10), 5 \mathrm{p}^{5} 4 \mathrm{f}^{2}$, and $5 \mathrm{p}^{5} 6 \mathrm{p}^{2}$ configurations outside the core [Cd] for calculation A, and nd, ng $(n=5-25)$, ns $(n=$ 6-24), nf $(n=4-22)$, and $\mathrm{np}(n=6-25)$ configurations outside the core $[\mathrm{Xe}]$ for calculation $\mathrm{B}$. Table 1 shows the wavelengths, $\lambda$ (in $\AA$ ); the weighted oscillator strengths, $g f$; the weighted transition rates (or probabilities), $g A_{k i}$ (in s ${ }^{-1}$ ), for nd $(n=5-9)-\mathrm{nf}(n=4-8)$, nd $(n=5-9)-\mathrm{np}(n=6-9)$, np $(n=6-9)-\mathrm{ns}(n=6-10)$, and ng $(n=5-8)-\mathrm{nf}(n=4-$ $8)$ electric dipole $(E 1)$ transitions. The data obtained are too much. For this reason, we have here presented just a part of the results. The comparing values for these exist in literature. Therefore, it is also made a comparison with other calculations and experiments in Table 1. We have also reported the wavelengths, the weighted oscillator strengths, and the weighted transition probabilities that are greater than or equal to $10^{5}$ for some new transitions $(680 \AA \leq \lambda \leq 8600 \AA)$ in Table 2. References for other comparison values are indicated below the tables with a lowercase superscript; oddparity states are indicated by the superscript " 0 ".

Electron correlation effects and relativistic effects play an important role in the spectra of heavy elements. To accurately predict the radiative atomic properties for heavy atoms such as La III, complex configuration interactions and relativistic effects must be considered simultaneously. Although Cowan's approach is based on Schrödinger's equation, it includes the most important relativistic effects like massvelocity corrections and Darwin contributions. Also, for complex atoms, it is important to allow for spin-orbit interaction, which represents the magnetic interaction energy between electron's spin magnetic moment and the magnetic field that the electron sees due to its orbital motion through the electric field of the nucleus. These contributions are considered as perturbations. Thus, to solve the Schrödinger equation with this Hamiltonian, we define a new angular momentum operator in an intermediate coupling scheme.

In calculations, the eigenvalues of Hamiltonian were optimized to the observed energy levels via a least-squares fitting procedure using experimentally determined energy levels, specifically all of the levels from the NIST compilation [28]. The scaling factors of the Slater parameters $\left(F^{k}\right.$ and $\left.G^{k}\right)$ and of configuration interaction integrals $\left(R^{k}\right)$, not optimized in the least-squares fitting, were chosen equal to 0.85 , while the spin-orbit parameters were left at their initial values. This low value of the scaling factors has been suggested by Cowan for neutral heavy elements $[15,16]$.

We obtained 7785 and 4278 possible E1 transitions between odd- and even-parity levels in the calculations A and $\mathrm{B}$, respectively. The results obtained are in excellent agreement with those of other works except some transitions. For some transitions, although the agreement is less in 
the weighted oscillator strengths and the weighted transition probabilities, it is very good in the wavelengths. Most of results related to low-lying levels obtained from this work are in agreement with literature [1-6]. The differences between our HFR results and other works for $g f$ and $g A_{k i}$ have been found in the $0-10 \%$ range for the transitions np $(n=6-8)-n s$ ( $n=6-10)$, nd $(n=6-9)$, in the $0.5-9 \%$ range for the transitions nd $(n=6,7)-\mathrm{nf}(n=5-8), \mathrm{np}(n=7-9)$, and in the $1.5-20 \%$ range for the transitions $\mathrm{nf}(n=5-8)-$ nd $(n=7-9)$, ng $(n=5-8)$. But the agreement is less in the weighted oscillator strengths and the weighted transition probabilities for $5 \mathrm{~d}$ and $4 \mathrm{f}$ transitions. In fact, except the transitions $6 \mathrm{p}$ ${ }^{2} \mathrm{P}_{3 / 2}^{\mathrm{o}}-9 \mathrm{~d}^{2} \mathrm{D}_{3 / 2}, 5 \mathrm{~d}^{2} \mathrm{D}_{3 / 2}-9 \mathrm{p}{ }^{2} \mathrm{P}_{1 / 2}^{\mathrm{o}}, 4 \mathrm{f}^{2} \mathrm{~F}_{7 / 2}^{\mathrm{o}}-5 \mathrm{~g}^{2} \mathrm{G}_{7 / 2}, 4 \mathrm{f}^{2} \mathrm{~F}_{7 / 2}^{\mathrm{o}}-$ $6 \mathrm{~g}^{2} \mathrm{G}_{7 / 2}$, and $4 \mathrm{f}^{2} \mathrm{~F}_{5 / 2}^{\mathrm{o}}-8 \mathrm{~g}{ }^{2} \mathrm{G}_{7 / 2,9 / 2}$, we found the values 1.064 (in calculation A) and 1.078 (in calculation B) for the mean ratio $g f$ (this work) $/ g f[1]$. Except the transitions $5 \mathrm{~d}-9 \mathrm{p}, 4 \mathrm{f}-$ $7 \mathrm{~g}, 8 \mathrm{~g}, 8 \mathrm{~s}-9 \mathrm{p}$, and $4 \mathrm{f}-6 \mathrm{~d}$, we found also the values 1.084 (in calculation A) and 1.126 (in calculation $B$ ) for the mean ratio $g A_{k i}$ (this work) $/ g A_{k i}$ [1]. The transition results obtained from the calculation A agree with other works. This calculation includes core correlation (including excitation from 5p shell in core). These results obtained from HFR calculations may be better in case that the increasing number of configurations including the excitations from core. It is noted that there are no exist the works, especially experimental, on La III recently in available literature. A detailed comparison needs new experimental works. Most of our results are excellent in agreement, expect the transition results to $4 \mathrm{f}$ and $5 \mathrm{~d}$ levels (for $g f$ and $g A_{k i}$ results), generally. It is well known that these levels interact strongly with core.

In conclusion, the main purpose of this paper was to perform HFR calculations for obtaining the description of La III spectrum. Accurate atomic structure data is an essential ingredient for a wide range of research fields. Areas from plasma research applications in nuclear fusion to lighting research, as well as astrophysics and cosmology, depend on such data. In spectrum synthesis works, particularly for CP stars, accurate data for transition probabilities (rates) and oscillator strengths for lanthanide atoms are needed to establish reliable abundances for these species. The agreement is excellent, especially for wavelengths, when our HFR results are compared with other available works in literature for the radiative transitions for La III. So, we may mention that new results presented in Table 2 for the transitions between some highly levels in this work are also reliable. There are a few experimental or theoretical radiative transition data for La III in literature. Consequently, we hope that our results, especially the new results in Table 2 , which are obtained using the HFR method will be useful for research fields, technological applications, and other works in the future for La III spectra.

\section{Acknowledgment}

The authors are very grateful to the anonymous reviewer for stimulating comments and valuable suggestions, which resulted in improvements to this paper.

\section{References}

[1] E. Biémont, Z. S. Li, P. Palmeri, and P. Quinet, "Radiative lifetimes in La III and oscillator strengths in La III and Lu III," Journal of Physics B, vol. 32, no. 14, pp. 3409-3419, 1999.

[2] H. Odabasi, "Spectrum of doubly ionized lanthanum (La III)," Journal of the Optical Society of America, vol. 57, no. 12, pp. 1459-1463, 1967.

[3] J. Migdalek and M. Wyrozumska, "Relativistic oscillator strengths for the Cs isoelectronic sequence and collapse of $\mathrm{f}$ and d orbitals," Journal of Quantitative Spectroscopy and Radiative Transfer, vol. 37, no. 6, pp. 581-589, 1987.

[4] J. Migdalek and W. E. Baylis, "Relativistic Hartree-Fock oscillator strengths for the lowest $s \rightarrow p$ transitions in the first few members of the $\mathrm{Rb}(\mathrm{I})$ and $\mathrm{Cs}(\mathrm{I})$ isoelectronic sequences, with allowance for core polarization," Journal of Quantitative Spectroscopy and Radiative Transfer, vol. 22, no. 2, pp. 127-134, 1979.

[5] S. Johansson and U. Litzén, "Resonance lines of La III," Journal of the Optical Society of America, vol. 61, no. 10, pp. 1427-1428, 1971.

[6] Z. S. Li and J. Zhankui, "Lifetime measurements in La II and La III using time-resolved laser spectroscopy," Physica Scripta, vol. 60, no. 5, pp. 414-417, 1999.

[7] E. Biémont and P. Quinet, "Recent advances in the study of lanthanide atoms and ions," Physica Scripta, vol. T105, pp. 3854, 2003.

[8] J. S. Badami, "The spectrum of trebly-ionized cerium (Ce IV)," Proceedings of the Physical Society, vol. 43, no. 1, pp. 53-58, 1931.

[9] J. Migdalek and A. Bojara, "Relativistic effects, core polarisation and relaxation in ionisation potentials along $\mathrm{Rb}$ and $\mathrm{Cs}$ isoelectronic sequences," Journal of Physics B, vol. 17, no. 10, pp. 1943-1951, 1984.

[10] P. Quinet and E. Biémont, "Landé g-factors for experimentally determined energy levels in doubly ionized lanthanides," Atomic Data and Nuclear Data Tables, vol. 87, no. 2, pp. 207230, 2004.

[11] R. C. Gibbs and H. E. White, "Relations between doublets of stripped atoms in five periods of the periodic table," Physical Review, vol. 33, no. 2, pp. 157-162, 1929.

[12] H. N. Russell and W. F. Meggers, "An analysis of lanthanum spectra (La I, La II, La III)," Journal of Research of the National Bureau of Standards, vol. 9, no. 5, pp. 625-668, 1932.

[13] J. Sugar and V. Kaufman, "Spectrum of doubly ionized lanthanum (La III)," Journal of the Optical Society of America, vol. 55, no. 10, pp. 1283-1285, 1965.

[14] B. Karaçoban and L. Özdemir, "Energies, Landé g-factors, and lifetimes for some excited levels of doubly ionized lanthanum," Central European Journal of Physics, vol. 10, no. 1, pp. 124-131, 2012.

[15] http://www.tcd.ie/Physics/People/Cormac.McGuinness/Cowan/.

[16] R. D. Cowan, The Theory of Atomic Structure and Spectra, California, USA, 1981.

[17] B. Karaçoban and L. Özdemir, "Energies and lifetimes for some excited levels in La I," Acta Physica Polonica A, vol. 113, no. 6, pp. 1609-1618, 2008.

[18] B. Karaçoban and L. Özdemir, "Electric dipole transitions for La I ( $Z$ = 57)," Journal of Quantitative Spectroscopy and Radiative Transfer, vol. 109, no. 11, pp. 1968-1985, 2008.

[19] B. Karaçoban and L. Özdemir, "The hyperfine structure calculations of some excited levels for ${ }^{(139)}$ La I," Acta Physica Polonica A, vol. 115, no. 5, pp. 864-872, 2009. 
[20] B. Karaçoban and L. Özdemir, "Transition energies of neutral and singly ionized lanthanum," Indian Journal of Physics, vol. 84 , no. 3, pp. 223-230, 2010.

[21] B. Karaçoban and L. Özdemir, "Electric dipole transitions for Lu I $(Z=71)$," Arabian Journal for Science and Engineering, vol. 36, no. 4, pp. 635-648, 2011.

[22] B. Karaçoban and L. Özdemir, "Energies and Landé factors for some excited levels in Lu I $(Z=71)$," Central European Journal of Physics, vol. 9, no. 3, pp. 800-806, 2011.

[23] B. Karaçoban and L. Özdemir, "Energies, Landé factors, and lifetimes for some excited levels of neutral ytterbium $(\mathrm{Z}=$ 70)," Acta Physica Polonica A, vol. 119, no. 3, pp. 342-353, 2011.

[24] B. Karaçoban and L. Özdemir, "Electric dipole transitions for neutral ytterbium $(Z=70)$," Journal of the Korean Physical Society, vol. 58, no. 3, pp. 417-428, 2011.

[25] B. Karaçoban and L. Özdemir, "Transition energies of ytterbium $(Z=70)$," Zeitschrift für Naturforschung A, vol. 66, pp. 543-551, 2011.

[26] B. Karaçoban and L. Özdemir, "The level structure of atomic lutetium $(Z=71)$ : a relativistic Hartree-Fock calculation," Indian Journal of Physics, vol. 85, no. 5, pp. 683-702, 2011.

[27] B. Karaçoban and L. Özdemir, "Transition energies of lutetium," Chinese Journal of Physics, vol. 50, no. 1, pp. 40-49, 2012.

[28] NIST, http://www.nist.gov/pml/data/asd.cfm. 

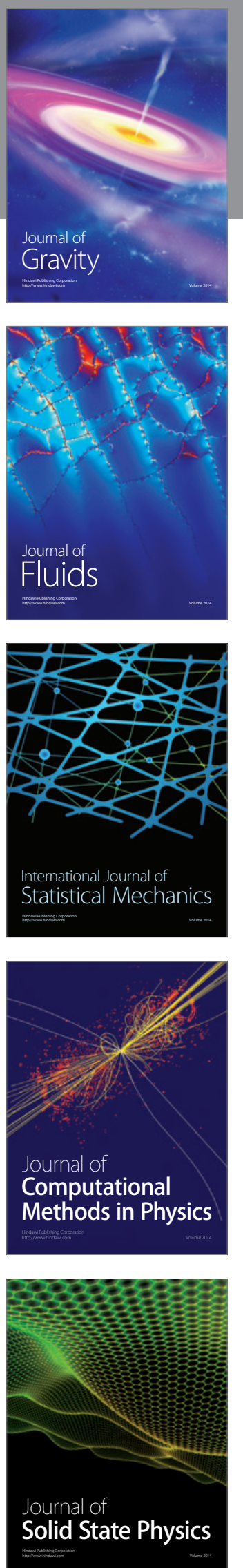

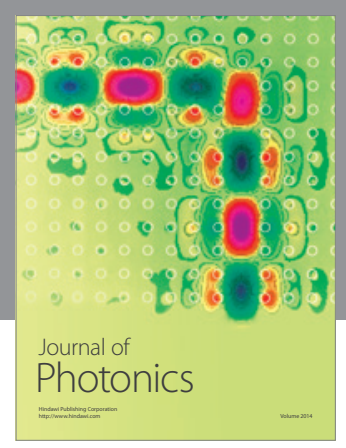

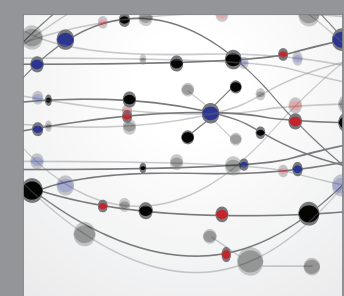

The Scientific World Journal
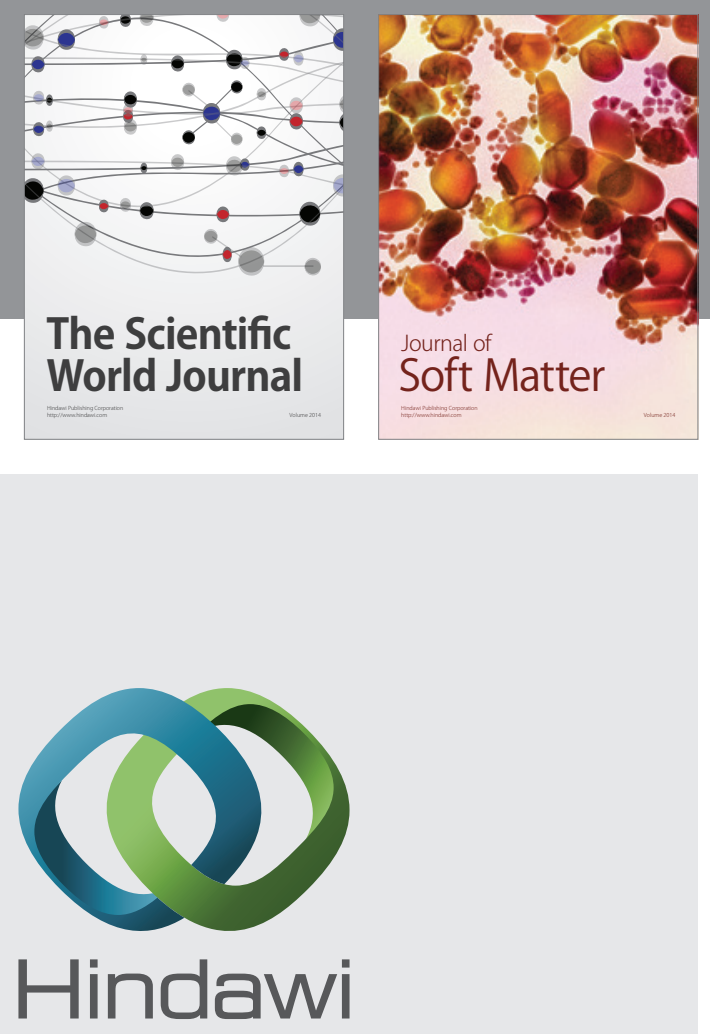

Submit your manuscripts at

http://www.hindawi.com
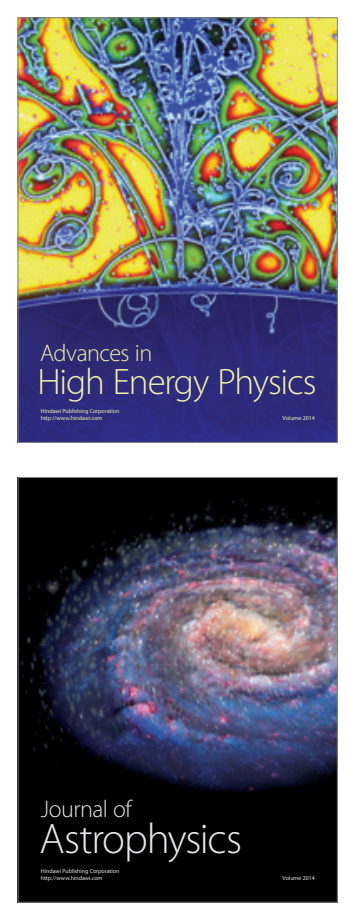
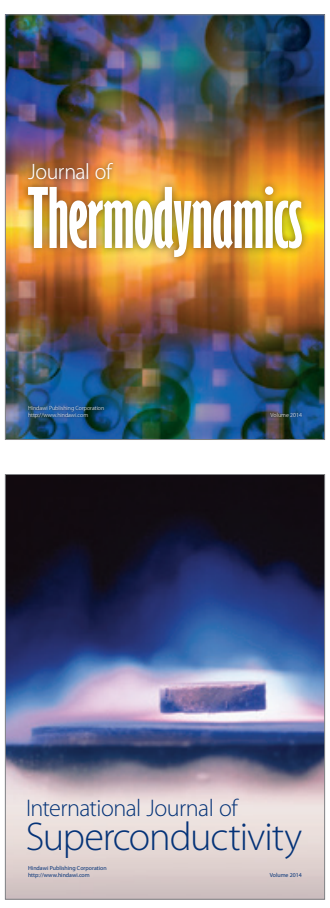
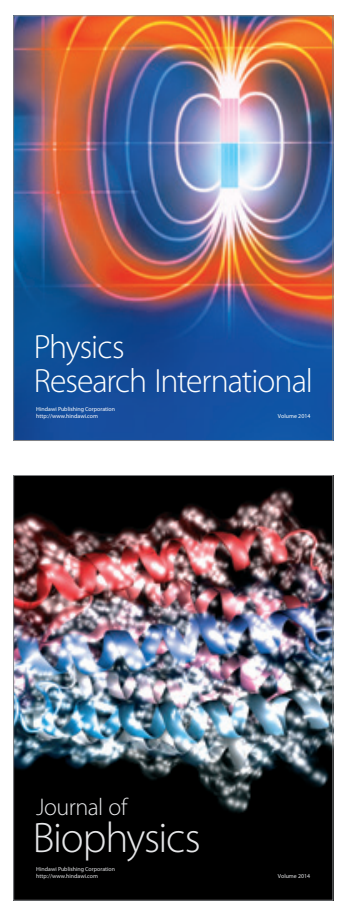
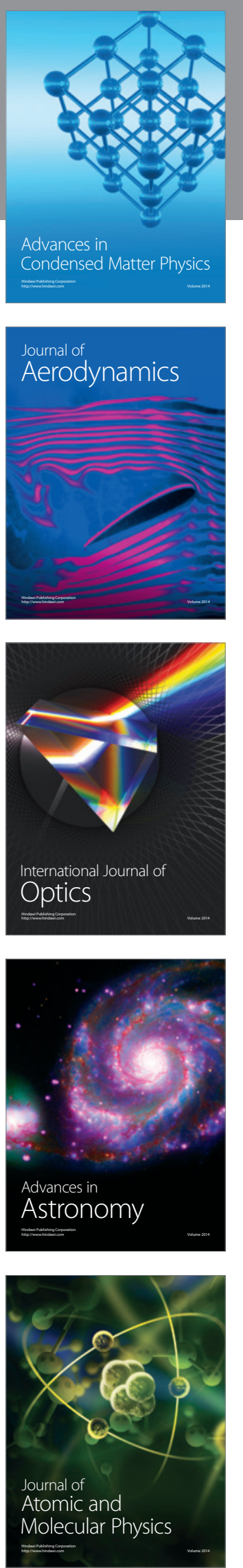